\title{
New Reticular Chemistry of the Rod Secondary Building Unit: Synthesis, Structure and Natural Gas Storage of a Series of 3-Way Rod Amide-Functionalized Metal-Organic Frameworks
}

Yu-Feng Zhang, ${ }^{\text {a } Z o n g-H u i ~ Z h a n g, ~}{ }^{a}$ Logan Ritter, ${ }^{\mathrm{b}}$ Han Fang, ${ }^{\mathrm{a}}$ Qian Wang, ${ }^{\mathrm{a}}$ Brian Space, ${ }^{\mathrm{b}}$ Yue-Biao Zhang, ${ }^{\mathrm{c}}$ Dong-Xu Xue, ${ }^{* a}$ and Junfeng Bai*a

${ }^{a}$ Key Laboratory of Applied Surface and Colloid Chemistry, Ministry of Education, Xi'an Key Laboratory of Organometallic Material Chemistry, School of Chemistry \& Chemical Engineering, Shaanxi Normal University, Xi'an 710062, China

${ }^{b}$ Department of Chemistry, University of South Florida 4202 E. Fowler Avenue, Tampa, Florida 33620, United States

${ }^{c}$ School of Physical Science and Technology, ShanghaiTech University, Shanghai 201210, China 


\section{Table of Contents}

Section 1. Materials and Methods............................................ 3

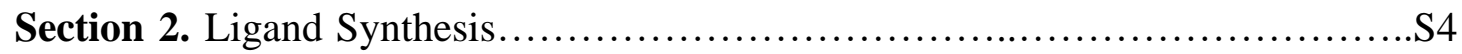

Section 3. Synthetic Procedures for 3W-ROD-1 and 3W-ROD-2-X..............S9

Section 4. Additional Structural Figures and Crystal Images....................S10

Section 5. PXRD and TGA Plots............................................ 32

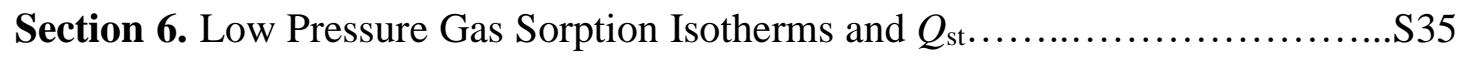

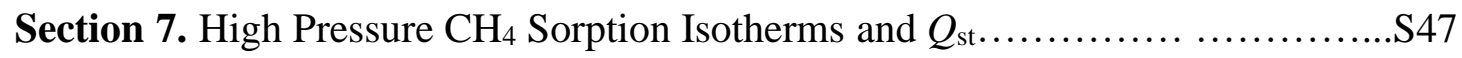

Section 8. Computational Methods......................................... 58

Section 9. Single-Crystal X-Ray Crystallography............................S66

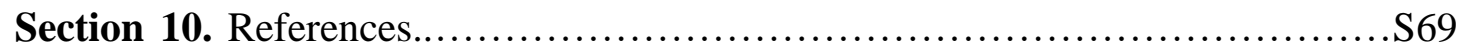




\section{Section 1. Materials and Methods.}

All reagents were obtained from commercial sources and used without further purification. Elemental analyses $(\mathrm{C}, \mathrm{H}$, and $\mathrm{N})$ were obtained from a Vario EL cube analyzer. PXRD measurements were performed on a Rigaku MiniFlex 600 diffractometer with $\mathrm{Cu} K \alpha(\lambda=1.5418 \AA)$, and the X-ray tube was operated at $40 \mathrm{kV}$ and $40 \mathrm{~mA}$. High resolution thermogravimetric analysis (TGA) were performed under a continuous $\mathrm{N}_{2}$ flow and recorded on a Q600SDT thermal analyzer with a heating rate of $5^{\circ} \mathrm{C}$ per minute. Fourier-transform infrared (FT-IR) spectrum $\left(4000-400 \mathrm{~cm}^{-1}\right.$, $\mathrm{KBr}$ pellet) was collected in the solid state on a Bruker Tensor 27 FT-IR spectrometer. The morphologies of the samples were obtained on a Desktop Scanning Electron Scanning Electron Microscope (TM300). 


\section{Section 2. Ligand Synthesis.}

The organic linker $\mathrm{H}_{3} \mathrm{~L}_{1}$ (4,4',4"-((benzene-1,3,5-tricarbonyl)tris(azanediyl))tribenzoic acid, $\mathrm{H}_{3} \mathrm{BTCB}$ ) was synthesized according to the literature procedure, ${ }^{1}$ and then $\mathrm{H}_{3} \mathrm{~L}_{1^{-}}$ $(\mathrm{OH})_{3}, \mathrm{H}_{3} \mathrm{~L}_{1}-\mathrm{F}_{3}, \mathrm{H}_{3} \mathrm{~L}_{1}-(\mathrm{Cl})_{3}$ and $\mathrm{H}_{3} \mathrm{~L}_{1}-\left(\mathrm{CH}_{3}\right)_{3}$ as well as $\mathrm{H}_{2} \mathrm{~L}_{2}-\mathrm{X}\left(\mathrm{X}=-\mathrm{OH},-\mathrm{F},-\mathrm{CH}_{3}\right)$ were synthesized as follows.

\section{Synthesis of $\quad 4,4^{\prime}, 4^{\prime \prime}$-((benzene-1,3,5-tricarbonyl)tris(azanediyl))tris(2-} hydroxybenzoic acid) ( $\left.\mathrm{H}_{3} \mathrm{~L}_{1}-(\mathrm{OH})_{3}\right)$.

$3.98 \mathrm{~g}$ (15.0 mmol) of 1,3,5-benzenetricarboxylic acid chloride was added to a solution of $6.90 \mathrm{~g}(45.1 \mathrm{mmol})$ of 4-amino-2-hydroxybenzoic acid and $3.62 \mathrm{~mL}$ (26.0 mmol) of triethylamine in $80 \mathrm{~mL}$ of $\mathrm{N}, \mathrm{N}$-dimethylacetamide (DMA). The mixture was stirred for $16 \mathrm{~h}$ at room temperature. After $300 \mathrm{~mL}$ of water had been added to the DMA solution, the resulting precipitate was collected by filtration, followed by washing with acetone, a large amount of water and methanol. The product was then air-dried. Yield $=8.60 \mathrm{~g}, 93.3 \% .{ }^{1} \mathrm{H}$ NMR $\left(400 \mathrm{MHz}, \mathrm{DMSO}-d_{6}\right): \delta=10.80(\mathrm{~s}, 3 \mathrm{H}$, $\mathrm{CON} \underline{\mathrm{H}}), 8.71(\mathrm{~s}, 3 \mathrm{H}, \mathrm{Ar} \underline{\mathrm{H}}), 7.82(\mathrm{~d}, 3 \mathrm{H}, \mathrm{Ar} \underline{\mathrm{H}}), 7.57$ (s, 3H, Arg $)$ and $7.38 \mathrm{ppm}(\mathrm{d}, 3 \mathrm{H}$, $\operatorname{Ar} \underline{\mathrm{H}})$.

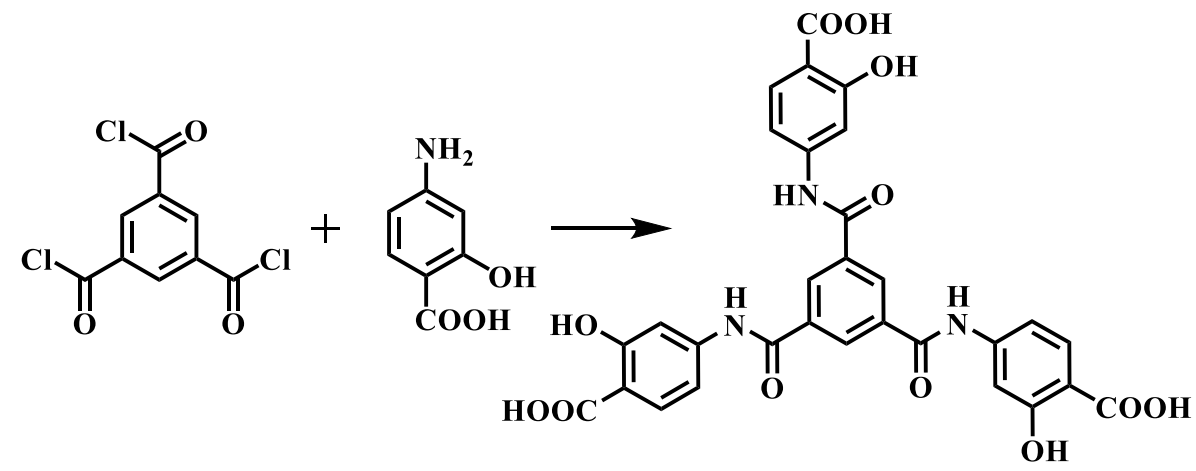

Scheme S1. Synthetic procedure for $\mathrm{H}_{3} \mathrm{~L}_{1}-(\mathrm{OH}) 3$.

\section{Synthesis of $\quad 4,4^{\prime}, 4^{\prime \prime}-(($ benzene-1,3,5-tricarbonyl)tris(azanediyl))tris(2- fluorobenzoic acid) $\left(\mathrm{H}_{3} \mathrm{~L}_{1}-\mathrm{F}_{3}\right)$.}

$3.98 \mathrm{~g}$ (15.0 mmol) of 1,3,5-benzenetricarboxylic acid chloride was added to a solution of $6.99 \mathrm{~g}(45.1 \mathrm{mmol})$ of 4-amino-2-fluorobenzoic acid and $3.62 \mathrm{~mL}(26.0$ $\mathrm{mmol}$ ) of triethylamine in $80 \mathrm{~mL}$ of $\mathrm{N}, \mathrm{N}$-dimethylacetamide (DMA). The mixture was stirred for $16 \mathrm{~h}$ at room temperature. After $300 \mathrm{~mL}$ of water had been added to the DMA solution, the resulting precipitate was collected by filtration, followed by washing with acetone, a large amount of water and methanol. The product was then air-dried. Yield $=8.80 \mathrm{~g}, 94.4 \% .{ }^{1} \mathrm{H}$ NMR $\left(400 \mathrm{MHz}, \mathrm{DMSO}-d_{6}\right): \delta=13.08(\mathrm{~s}, 3 \mathrm{H}$, $\mathrm{COO} \underline{\mathrm{H}}), 11.09$ (s, 3H, CON$\underline{\mathrm{H}}), 8.78$ (s, 3H, Ar$\underline{\mathrm{H}}), 7.93$ (dd, 6H, Ar$\underline{\mathrm{H}})$ and $7.74 \mathrm{ppm}$ (d, $3 \mathrm{H}, \mathrm{Ar} \underline{\mathrm{H}})$. 


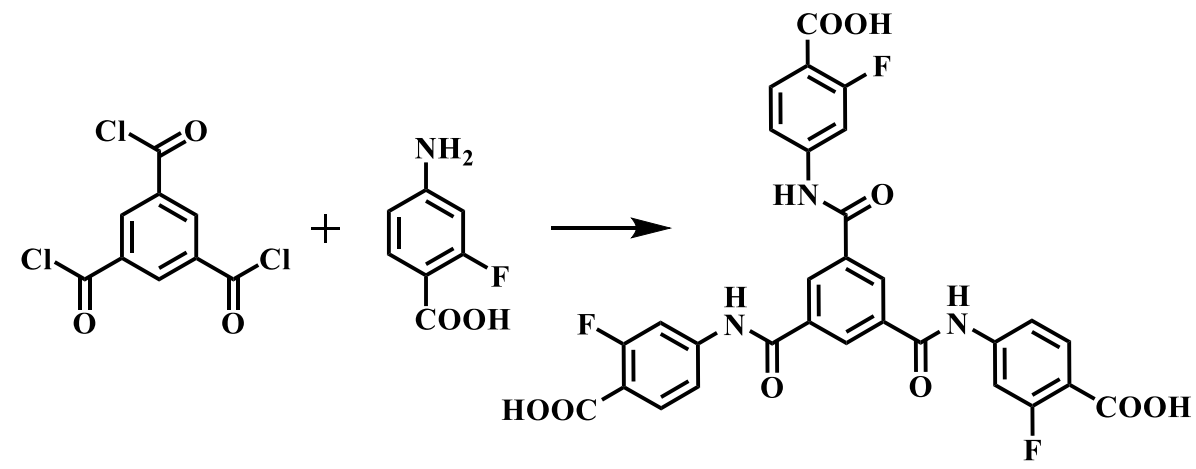

Scheme S2. Synthetic procedure for $\mathrm{H}_{3} \mathrm{~L}_{1}-\mathrm{F}_{3}$.

\section{Synthesis of $\quad 4,4^{\prime}, 4^{\prime \prime}$-((benzene-1,3,5-tricarbonyl)tris(azanediyl))tris(2- chlorobenzoic acid) $\left(\mathrm{H}_{3} \mathrm{~L}_{1}-(\mathrm{Cl})_{3}\right)$.}

$3.98 \mathrm{~g}$ (15.0 mmol) of 1,3,5-benzenetricarboxylic acid chloride was added to a solution of $7.71 \mathrm{~g}(45.1 \mathrm{mmol})$ of 4-amino-2-chlorobenzoic acid and $3.62 \mathrm{~mL}(26.0$ mmol) of triethylamine in $80 \mathrm{~mL}$ of $\mathrm{N}, \mathrm{N}$-dimethylacetamide (DMA). The mixture was stirred for $16 \mathrm{~h}$ at room temperature. After $300 \mathrm{~mL}$ of water had been added to the DMA solution, the resulting precipitate was collected by filtration, followed by washing with acetone, a large amount of water and methanol. The product was then air-dried. Yield $=9.30 \mathrm{~g}, 92.5 \%$. ${ }^{1} \mathrm{H}$ NMR (400 MHz, DMSO- $\left.d_{6}\right): \delta=13.22(\mathrm{~s}, 3 \mathrm{H}$,

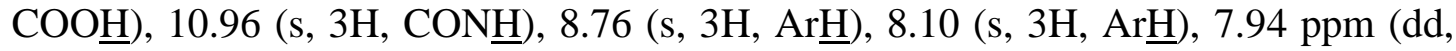
$6 \mathrm{H}, \mathrm{Ar} \underline{\mathrm{H}})$.<smiles>CC(C)(C)C(=O)c1cc(C(=O)Nc2ccc(C(=O)O)c(Cl)c2)cc(C(=O)Nc2ccc(C(=O)O)c(Cl)c2)c1</smiles>

Scheme S3. Synthetic procedure for $\mathrm{H}_{3} \mathrm{~L}_{1}-(\mathrm{Cl})_{3}$.

\section{Synthesis of 4,4',4'-((benzene-1,3,5-tricarbonyl)tris(azanediyl))tris(2- methylbenzoic acid)) ( $\left.\mathrm{H}_{3} \mathrm{~L}_{1}-\left(\mathrm{CH}_{3}\right)_{3}\right)$.}

$3.98 \mathrm{~g}(15.0 \mathrm{mmol})$ of 1,3,5-benzenetricarboxylic acid chloride was added to a solution of $6.81 \mathrm{~g}(45.1 \mathrm{mmol})$ of 4-amino-2-methylbenzoic acid and $3.62 \mathrm{~mL}(26.0$ mmol) of triethylamine in $80 \mathrm{~mL}$ of $\mathrm{N}, \mathrm{N}$-dimethylacetamide (DMA). The mixture was stirred for $16 \mathrm{~h}$ at room temperature. After $300 \mathrm{~mL}$ of water had been added to the DMA solution, the resulting precipitate was collected by filtration, followed by washing with acetone, a large amount of water and methanol. The product was then air-dried. Yield $=8.0 \mathrm{~g}, 87.6 \% .{ }^{1} \mathrm{H}$ NMR $\left(400 \mathrm{MHz}, \mathrm{DMSO}-d_{6}\right): \delta=12.68(\mathrm{~s}, 3 \mathrm{H}$, 
$\mathrm{COOH}), 10.75(\mathrm{~s}, 3 \mathrm{H}, \mathrm{CONH}), 8.73(\mathrm{~s}, 3 \mathrm{H}, \operatorname{Ar} \underline{\mathrm{H}}), 7.92(\mathrm{~d}, 3 \mathrm{H}, \operatorname{Ar} \underline{\mathrm{H}}), 7.81(\mathrm{~d}, 3 \mathrm{H}$,

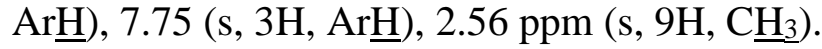

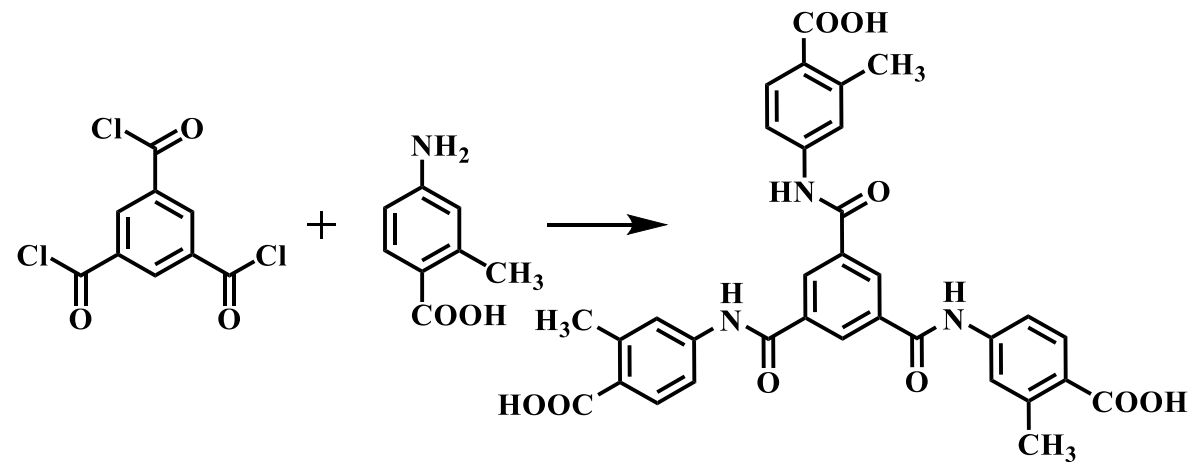

Scheme S4. Synthetic procedure for $\mathrm{H}_{3} \mathrm{~L}_{1}-\left(\mathrm{CH}_{3}\right)_{3}$.

Synthesis of 4-(4-carboxybenzamido)-2-hydroxybenzoic acid $\left(\mathrm{H}_{2} \mathrm{~L}_{2}-\mathrm{OH}\right)$.

4-Aminosalicylic acid $(6.12 \mathrm{~g}, 40.0 \mathrm{mmol})$ was mixed with diethyl ether $(75 \mathrm{~mL})$ and cooled to $5{ }^{\circ} \mathrm{C}$ in an ice bath. 4-(chlorocarbonyl) benzoate $(7.94 \mathrm{~g}, 40.0 \mathrm{mmol})$ was then added slowly. The reaction mixture was stirred at $5{ }^{\circ} \mathrm{C}$ for $4 \mathrm{~h}$, followed by continuous stirring at room temperature for an additional $20 \mathrm{~h}$. At the end of this period, distilled water $(100 \mathrm{~mL})$ was added to the reaction. The product was filtered and washed with DMF $(2 \times 50 \mathrm{~mL})$, distilled water $(5 \times 150 \mathrm{~mL})$, and $\mathrm{MeOH}(2 \times$ $100 \mathrm{~mL}$ ). The product was then dried in air to afford the $\mathrm{H}_{2} \mathrm{~L}-\mathrm{OH}$ ester as a light grey powder (7.96 g, $26.6 \mathrm{mmol}, 66 \%$ yield). ${ }^{1} \mathrm{H}$ NMR (400 MHz, DMSO- $\left.d 6\right): \delta=13.72$ (s, $1 \mathrm{H}, \mathrm{COO} \underline{\mathrm{H}}), 11.37(\mathrm{~s}, 1 \mathrm{H}, \mathrm{OH}), 10.65(\mathrm{~s}, 1 \mathrm{H}, \mathrm{CON} \underline{\mathrm{H}}), 8.08$ (dd, 4H, Ar$\underline{\mathrm{H}}), 7.77$ (d, $1 \mathrm{H}, \operatorname{Ar} \underline{\mathrm{H}}), 7.54(\mathrm{~s}, 1 \mathrm{H}, \mathrm{Ar} \underline{\mathrm{H}}), 7.33(\mathrm{~d}, 1 \mathrm{H}, \mathrm{Ar} \underline{\mathrm{H}})$ and $3.91 \mathrm{ppm}\left(\mathrm{s}, 3 \mathrm{H}, \mathrm{C}_{3}\right)$.

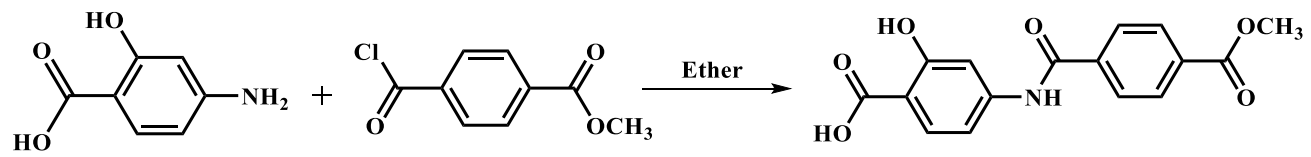

Scheme S5. Synthetic procedure for $\mathrm{H}_{2} \mathrm{~L}_{2}-\mathrm{OH}$ ester.

A solution containing $\mathrm{H}_{2} \mathrm{~L}_{2}-\mathrm{OH}$ ester $(3.98 \mathrm{~g}, 13.28 \mathrm{mmol}), \mathrm{NaOH}(1.08 \mathrm{~g}, 27 \mathrm{mmol})$, ethanol $(120 \mathrm{~mL})$ and $\mathrm{H}_{2} \mathrm{O}(120 \mathrm{~mL})$ was prepared and subject to reflux overnight. The resulting transparent solution was cooled to room temperature and acidified with a $71.5 \mathrm{~mL} 2 \mathrm{M} \mathrm{HCl}$ and $71.5 \mathrm{~mL} \mathrm{H}_{2} \mathrm{O}$ mixture, forming a grey suspension. The reaction mixture was sealed and allowed to stir for 3 hours. The product was collected by filtration and washed copiously with water until washings were neutral. The grey powder was added to a sample vial which was dried under air $(3.58 \mathrm{~g}, 11.89 \mathrm{mmol}$, 89\%). ${ }^{1} \mathrm{H}$ NMR (400 MHz, DMSO- $d 6$ ): $\delta=13.30$ (s, $1 \mathrm{H}, \mathrm{COO} \underline{\mathrm{H}}$ ), 11.38 (s, $1 \mathrm{H}, \mathrm{OH}$ ),

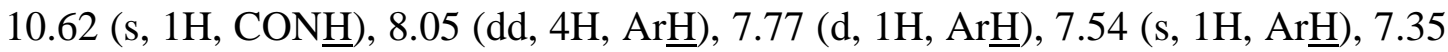
(d, 1H, Ar $\underline{\mathrm{H}})$.

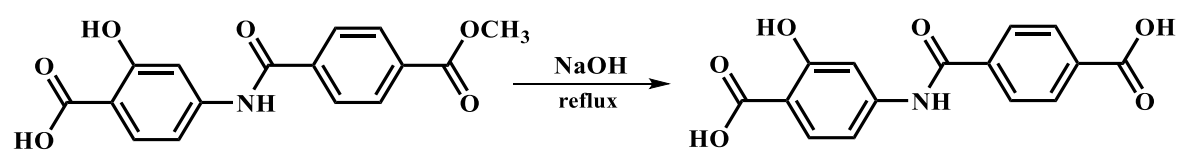

Scheme S6. Synthetic procedure for $\mathrm{H}_{2} \mathrm{~L}_{2}-\mathrm{OH}$. 


\section{Synthesis of 4-(4-carboxybenzamido)-2-fluorobenzoic acid $\left(\mathbf{H}_{2} \mathbf{L}_{2}-\mathrm{F}\right)$}

4-amino-2-fluorobenzoic acid (6.20 g, $40.0 \mathrm{mmol})$ was mixed with diethyl ether (75 $\mathrm{mL}$ ) and cooled to $5{ }^{\circ} \mathrm{C}$ in an ice bath. 4-(chlorocarbonyl) benzoate $(7.94 \mathrm{~g}, 40.0$ mmol) was then added slowly. The reaction mixture was stirred at $5{ }^{\circ} \mathrm{C}$ for $4 \mathrm{~h}$, followed by continuous stirring at room temperature for an additional $20 \mathrm{~h}$. At the end of this period, distilled water $(100 \mathrm{~mL})$ was added to the reaction. The product was filtered and washed with DMF $(2 \times 50 \mathrm{~mL})$, distilled water $(5 \times 150 \mathrm{~mL})$, and $\mathrm{MeOH}$ $(2 \times 100 \mathrm{~mL})$. The product was then dried in air to afford the $\mathrm{H}_{2} \mathrm{~L}-\mathrm{F}$ ester as a light yellow powder (7.60 g, $25.2 \mathrm{mmol}, 63 \%$ yield). ${ }^{1} \mathrm{H}$ NMR (400 MHz, DMSO- $\left.d 6\right): \delta=$

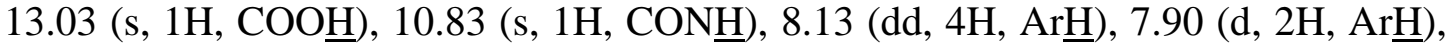
7.67 (s, $1 \mathrm{H}, \mathrm{Ar} \underline{\mathrm{H}})$, and $3.89 \mathrm{ppm}\left(\mathrm{s}, 3 \mathrm{H}, \mathrm{C}_{3}\right)$.

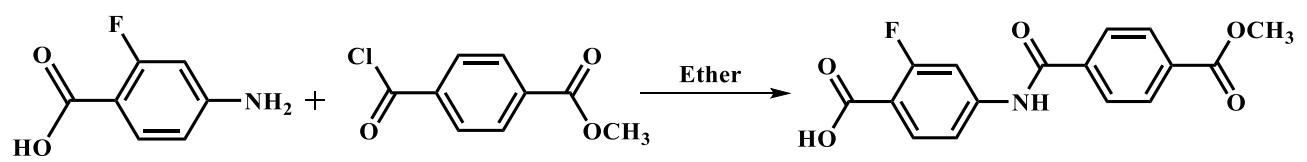

Scheme S7. Synthetic procedure for $\mathrm{H}_{2} \mathrm{~L}_{2}-\mathrm{F}$ ester.

A solution containing $\mathrm{H}_{2} \mathrm{~L}_{2}-\mathrm{F}$ ester (2.60 g, $\left.8.22 \mathrm{mmol}\right), \mathrm{NaOH}(0.67 \mathrm{~g}, 16.75 \mathrm{mmol})$, ethanol $(75 \mathrm{~mL})$ and $\mathrm{H}_{2} \mathrm{O}(75 \mathrm{~mL})$ was prepared and subject to reflux overnight. The resulting transparent solution was cooled to room temperature and acidified with a $44.35 \mathrm{~mL} 2 \mathrm{M} \mathrm{HCl}$ and $44.35 \mathrm{~mL} \mathrm{H}_{2} \mathrm{O}$ mixture, forming a yellow suspension. The reaction mixture was sealed and allowed to stir for 3 hours. The product was collected by filtration and washed copiously with water until washings were neutral. The light yellow powder was added to a sample vial which was dried under air (2.40 g, 7.92 mmol, 96\%). ${ }^{1} \mathrm{H}$ NMR (400 MHz, DMSO- $d 6$ ): $\delta=13.19$ (s, 2H, COOH ), 10.82 (s, 1H,

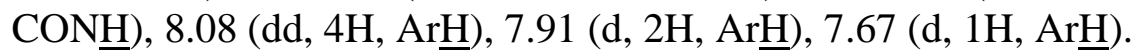

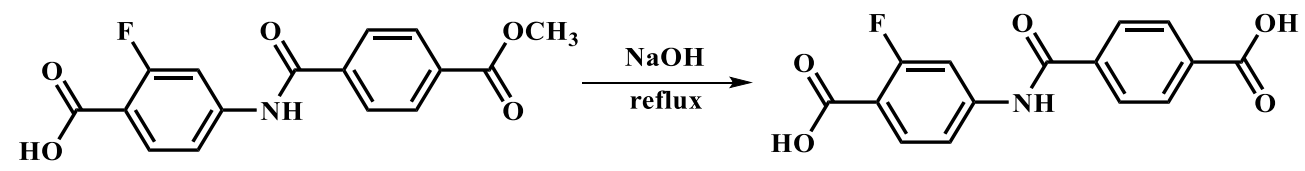

Scheme S8. Synthetic procedure for $\mathrm{H}_{2} \mathrm{~L}_{2}-\mathrm{F}$.

\section{Synthesis of 4-(4-carboxybenzamido)-2-methylbenzoic acid $\left(\mathrm{H}_{2} \mathrm{~L}_{2}-\mathrm{CH}_{3}\right)$.}

4-amino-2-methylbenzoic acid (6.04 g, $40.0 \mathrm{mmol})$ was mixed with diethyl ether (100 $\mathrm{mL}$ ) and cooled to $5{ }^{\circ} \mathrm{C}$ in an ice bath. 4-(chlorocarbonyl) benzoate (7.94 g, 40.0 mmol) was then added slowly. The reaction mixture was stirred at $5{ }^{\circ} \mathrm{C}$ for $4 \mathrm{~h}$, followed by continuous stirring at room temperature for an additional $20 \mathrm{~h}$. At the end of this period, distilled water $(100 \mathrm{~mL})$ was added to the reaction. The product was filtered and washed with DMF $(2 \times 50 \mathrm{~mL})$, distilled water $(5 \times 150 \mathrm{~mL})$, and $\mathrm{MeOH}$ $(2 \times 100 \mathrm{~mL})$. The product was then dried in air to afford the $\mathrm{H}_{2} \mathrm{~L}-\mathrm{CH}_{3}$ ester as a white powder ( $8.20 \mathrm{~g}, 26.20 \mathrm{mmol}, 65.5 \%$ yield). ${ }^{1} \mathrm{H}$ NMR (400 MHz, DMSO- $\left.d 6\right): \delta$ $=12.62(\mathrm{~s}, 1 \mathrm{H}, \mathrm{COO} \underline{\mathrm{H}}), 10.60(\mathrm{~s}, 1 \mathrm{H}, \mathrm{CON} \underline{\mathrm{H}}), 8.09(\mathrm{dd}, 4 \mathrm{H}, \mathrm{ArH}), 7.87(\mathrm{~d}, 1 \mathrm{H}, \operatorname{Ar} \underline{\mathrm{H}})$, $7.73(\mathrm{~d}, 2 \mathrm{H}, \mathrm{Ar} \underline{\mathrm{H}})$ and $3.90 \mathrm{ppm}\left(\mathrm{s}, 3 \mathrm{H}, \mathrm{C}_{3}\right)$. 


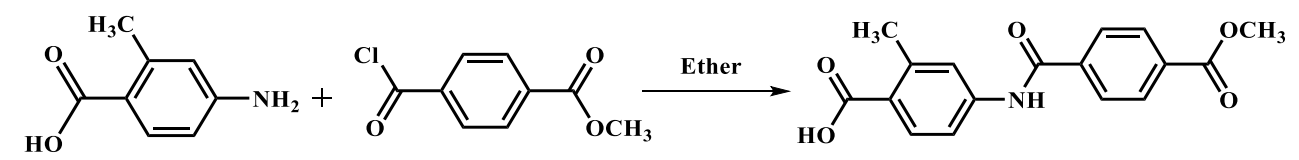

Scheme S9. Synthetic procedure for $\mathrm{H}_{2} \mathrm{~L}_{2}-\mathrm{CH}_{3}$ ester.

A solution containing $\mathrm{H}_{2} \mathrm{~L}-\mathrm{CH}_{3}$ ester $(2.57 \mathrm{~g}, 8.22 \mathrm{mmol}), \mathrm{NaOH}(0.67 \mathrm{~g}, 16.75$ mmol), ethanol $(40 \mathrm{~mL})$ and $\mathrm{H}_{2} \mathrm{O}(40 \mathrm{~mL})$ was prepared and subject to reflux overnight. The resulting transparent solution was cooled to room temperature and acidified with a $44.35 \mathrm{~mL} 2 \mathrm{M} \mathrm{HCl}$ and $44.35 \mathrm{~mL} \mathrm{H} \mathrm{H}_{2} \mathrm{O}$ mixture, forming a yellow suspension. The reaction mixture was sealed and allowed to stir for 3 hours. The product was collected by filtration and washed copiously with water until washings were neutral. The white powder was added to a sample vial which was dried under air (2.40 g, $8.03 \mathrm{mmol}, 98 \%)$. ${ }^{1} \mathrm{H}$ NMR (400 MHz, DMSO-d6): $\delta=12.93$ (s, 2H, COOH$)$, $10.56(\mathrm{~s}, 1 \mathrm{H}, \mathrm{CON} \underline{\mathrm{H}}), 8.06(\mathrm{dd}, 4 \mathrm{H}, \operatorname{Ar} \underline{\mathrm{H}}), 7.87$ (d, 1H, Ar$\underline{\mathrm{H}}), 7.73$ (d, 2H, Ar$\underline{\mathrm{H}})$.

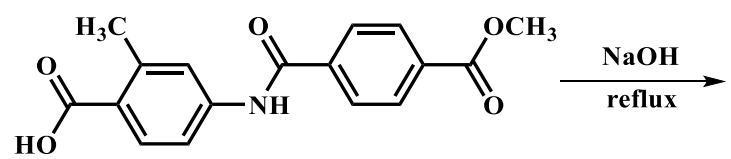<smiles>Cc1cc(NC(=O)c2ccc(C(=O)O)cc2)ccc1C(=O)O</smiles>

Scheme S10. Synthetic procedure for $\mathrm{H}_{2} \mathrm{~L}_{2}-\mathrm{CH}_{3}$. 
Section 3. Synthetic Procedures for 3W-ROD-1 and 3W-ROD-2-X

Synthesis of 3W-ROD-1: $\mathrm{H}_{3} \mathrm{~L}_{1}(21.26 \mathrm{mg}, 0.0375 \mathrm{mmol}), \mathrm{In}\left(\mathrm{NO}_{3}\right)_{3} \cdot 4 \mathrm{H}_{2} \mathrm{O}(26.2 \mathrm{mg}$, $0.087 \mathrm{mmol}), \mathrm{DMF}(3.0 \mathrm{~mL}), \mathrm{HNO}_{3}(0.5 \mathrm{~mL}, 7.0 \mathrm{M}$ in DMF) were combined in a 20 $\mathrm{mL}$ scintillation vial, sealed, and heated to $105^{\circ} \mathrm{C}$ for $48 \mathrm{~h}$ and then cooled to room temperature. The colorless polyhedral crystals were collected and washed several times by DMF. CHN elemental analysis (\%) for 3W-ROD-1, $\left[\mathrm{In}_{5}(\mathrm{OH})_{6}\left(\mathrm{C}_{30} \mathrm{H}_{18} \mathrm{~N}_{3} \mathrm{O}_{9}\right)_{3}\left(\mathrm{H}_{2} \mathrm{O}\right)\right] \cdot\left(\mathrm{H}_{2} \mathrm{O}\right)_{25}\left(\mathrm{C}_{3} \mathrm{H}_{7} \mathrm{NO}\right)_{9}$ : Calculated $\mathrm{C}$ (40.20), H (5.05), N (7.21); Found C (40.61), H (4.78), N (7.50). FT-IR: (KBr 4000-400 cm $\left.\mathrm{cm}^{-1}\right)$ : 3472 (br, w), 3281 (w), 1664 (vs), 1603 (m), 1525 (m), 1390 (m), 1312 (m), 1250 (s), 1096 (s), $1059(\mathrm{w}), 781(\mathrm{~s}), 655(\mathrm{~m}), 501(\mathrm{w}), 449(\mathrm{w})$.

Synthesis of 3W-ROD-2-OH: $\mathrm{H}_{3} \mathrm{~L}_{1}(12.3 \mathrm{mg}, 0.02175 \mathrm{mmol}), \mathrm{H}_{2} \mathrm{~L}_{2}-\mathrm{OH}(6.5 \mathrm{mg}$, $0.02175 \mathrm{mmol}), \mathrm{In}\left(\mathrm{NO}_{3}\right)_{3} \cdot 4 \mathrm{H}_{2} \mathrm{O}$ (13.0 mg, $\left.0.0435 \mathrm{mmol}\right), \mathrm{DMF}(2.5 \mathrm{~mL}), \mathrm{HNO}_{3}(0.3$ $\mathrm{mL}, 7.0 \mathrm{M}$ in DMF) were combined in a $20 \mathrm{~mL}$ scintillation vial, sealed, and heated to $105{ }^{\circ} \mathrm{C}$ for $36 \mathrm{~h}$ and then cooled to room temperature. The yellow polyhedral crystals were collected and washed several times by DMF. CHN elemental analysis (\%) for 3W-ROD-2-OH, $\quad\left[\mathrm{In}_{5}(\mathrm{OH})_{5}\left(\mathrm{C}_{30} \mathrm{H}_{18} \mathrm{~N}_{3} \mathrm{O}_{9}\right)_{8 / 3}\left(\mathrm{C}_{15} \mathrm{H}_{9} \mathrm{NO}_{6}\right)\right] \cdot\left(\mathrm{H}_{2} \mathrm{O}\right)_{30}\left(\mathrm{C}_{3} \mathrm{H}_{7} \mathrm{NO}\right)_{12}$ : Calculated C (40.54), H (5.35), N (7.58); Found C (40.28), H (5.22), N (7.68). FT-IR: (KBr 4000-400 cm cm $^{-1} 3453$ (br, w), 3288 (w), 1662 (vs), 1601 (m), 1530 (m), 1387 (m), 1307 (m), 1250 (s), 1095 (s), 1060 (w), 780 (s), 651 (m), 500 (w), 449 (w).

Synthesis of 3W-ROD-2-F: $\mathrm{H}_{3} \mathrm{~L}_{1}(11.3 \mathrm{mg}, 0.02 \mathrm{mmol}), \mathrm{H}_{2} \mathrm{~L}_{2}-\mathrm{F}(6.0 \mathrm{mg}, 0.02$ $\mathrm{mmol}), \mathrm{In}\left(\mathrm{NO}_{3}\right)_{3} \cdot 4 \mathrm{H}_{2} \mathrm{O}(13.0 \mathrm{mg}, 0.0435 \mathrm{mmol}), \mathrm{DMF}(2.5 \mathrm{~mL}), \mathrm{HNO}_{3}(0.25 \mathrm{~mL}, 7.0$ $\mathrm{M}$ in DMF) were combined in a $20 \mathrm{~mL}$ scintillation vial, sealed, and heated to $105^{\circ} \mathrm{C}$ for $36 \mathrm{~h}$ and then cooled to room temperature. The colorless polyhedral crystals were collected and washed several times by DMF. CHN elemental analysis (\%) for $3 \mathrm{~W}$ ROD-2-F, [ $\left.\mathrm{In}_{5}(\mathrm{OH})_{5}\left(\mathrm{C}_{30} \mathrm{H}_{18} \mathrm{~N}_{3} \mathrm{O}_{9}\right)_{8 / 3}\left(\mathrm{C}_{15} \mathrm{H}_{8} \mathrm{NFO}_{5}\right)\right] \cdot\left(\mathrm{H}_{2} \mathrm{O}\right)_{29}\left(\mathrm{C}_{3} \mathrm{H}_{7} \mathrm{NO}\right)_{12}$ : Calculated C (40.71), H (5.29), N (7.61); Found C (40.61), H (4.99), N (7.76). FT-IR: (KBr 4000$400 \mathrm{~cm}^{-1}$ ): 3280 (w), 1662 (vs), 1602 (m), 1523 (m), 1391 (m), 1309 (m), 1249 (s), 1097 (s), 1056 (w), 785 (s), 659 (m), 504 (w), 448 (w).

Synthesis of 3W-ROD-2-CH3: $\mathrm{H}_{3} \mathrm{~L}_{1}(11.3 \mathrm{mg}, 0.02 \mathrm{mmol}), \mathrm{H}_{2} \mathrm{~L}_{2}-\mathrm{CH}_{3}(6.0 \mathrm{mg}, 0.02$ $\mathrm{mmol}), \mathrm{In}\left(\mathrm{NO}_{3}\right)_{3} \cdot 4 \mathrm{H}_{2} \mathrm{O}(13.0 \mathrm{mg}, 0.0435 \mathrm{mmol}), \mathrm{DMF}(2.5 \mathrm{~mL}), \mathrm{HNO}_{3}(0.20 \mathrm{~mL}, 7.0$ $\mathrm{M}$ in DMF) were combined in a $20 \mathrm{~mL}$ scintillation vial, sealed, and heated to $105^{\circ} \mathrm{C}$ for $36 \mathrm{~h}$ and cooled to room temperature. The colorless polyhedral crystals were collected and washed several times by DMF. CHN elemental analysis (\%) for $3 \mathrm{~W}$ ROD-2- $\mathrm{CH}_{3},\left[\mathrm{In}_{5}(\mathrm{OH})_{5}\left(\mathrm{C}_{30} \mathrm{H}_{18} \mathrm{~N}_{3} \mathrm{O}_{9}\right)_{8 / 3}\left(\mathrm{C}_{16} \mathrm{H}_{11} \mathrm{NO}_{5}\right)\right] \cdot\left(\mathrm{H}_{2} \mathrm{O}\right)_{30}\left(\mathrm{C}_{3} \mathrm{H}_{7} \mathrm{NO}\right)_{10}$ : Calculated C (40.54), H (5.24), N (7.13); Found C (40.52), H (5.31), N (7.42). FT-IR: (KBr 4000-400 cm ${ }^{-1}$ ): 3281 (w), 1663 (vs), 1603 (m), $1523(\mathrm{~m}), 1389$ (m), 1312 (m), 1249 (s), 1095 (s), 1060 (w), 782 (s), $658(\mathrm{~m}), 507$ (w), $446(\mathrm{w})$. 
Section 4. Additional Structural Figures and Crystal Images

One-Way ROD MOFs
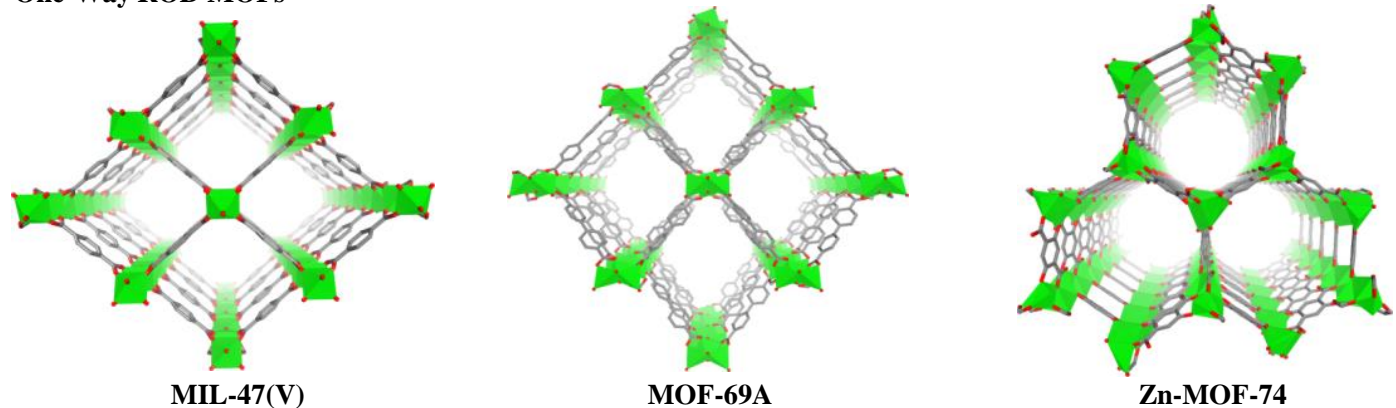

Two-Way ROD MOFs

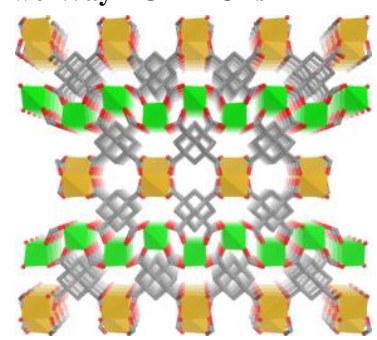

MOF-77

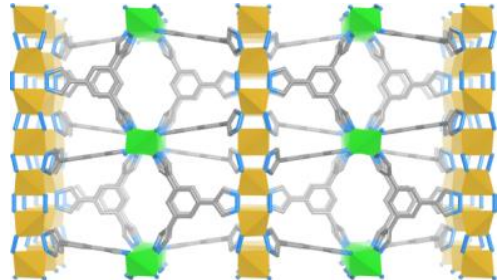

$\mathrm{Zn}_{3}(\mathbf{B T P})_{2}$

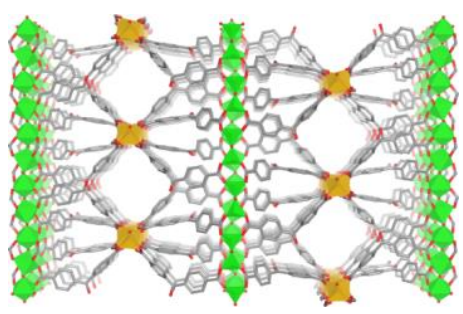

CAU-8

Three-Way ROD MOFs

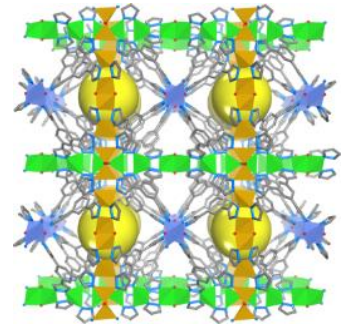

$\mathrm{Zn}_{6}\left(\mathrm{H}_{2} \mathrm{O}\right)_{3}(\mathrm{BTP})_{4}$

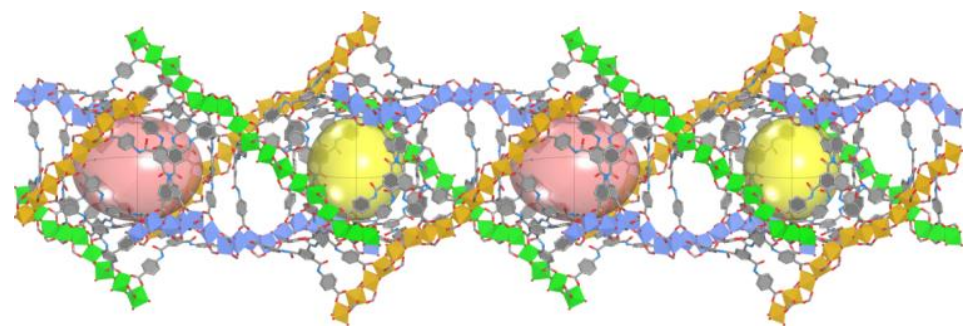

3W-ROD-1

Figure S1. The typical rod MOFs classified by the features of rod packing patterns. 


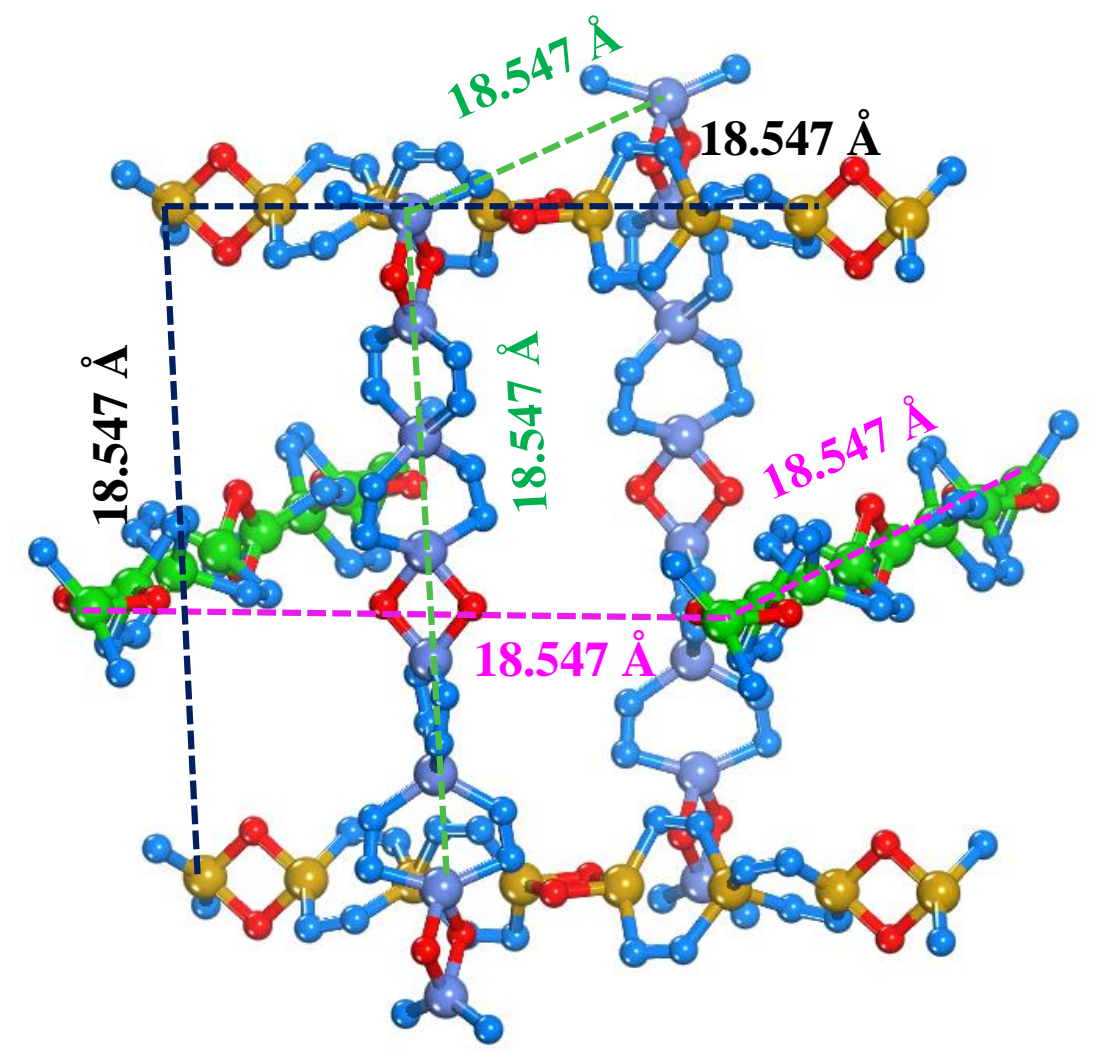

Figure S2. Schematic representation showing that spacing along the rods (translation) and between the rods in $\mathrm{Zn}_{6}\left(\mathrm{H}_{2} \mathrm{O}\right)_{3}(\mathrm{BTP})_{4}$ is $18.547 \AA$.

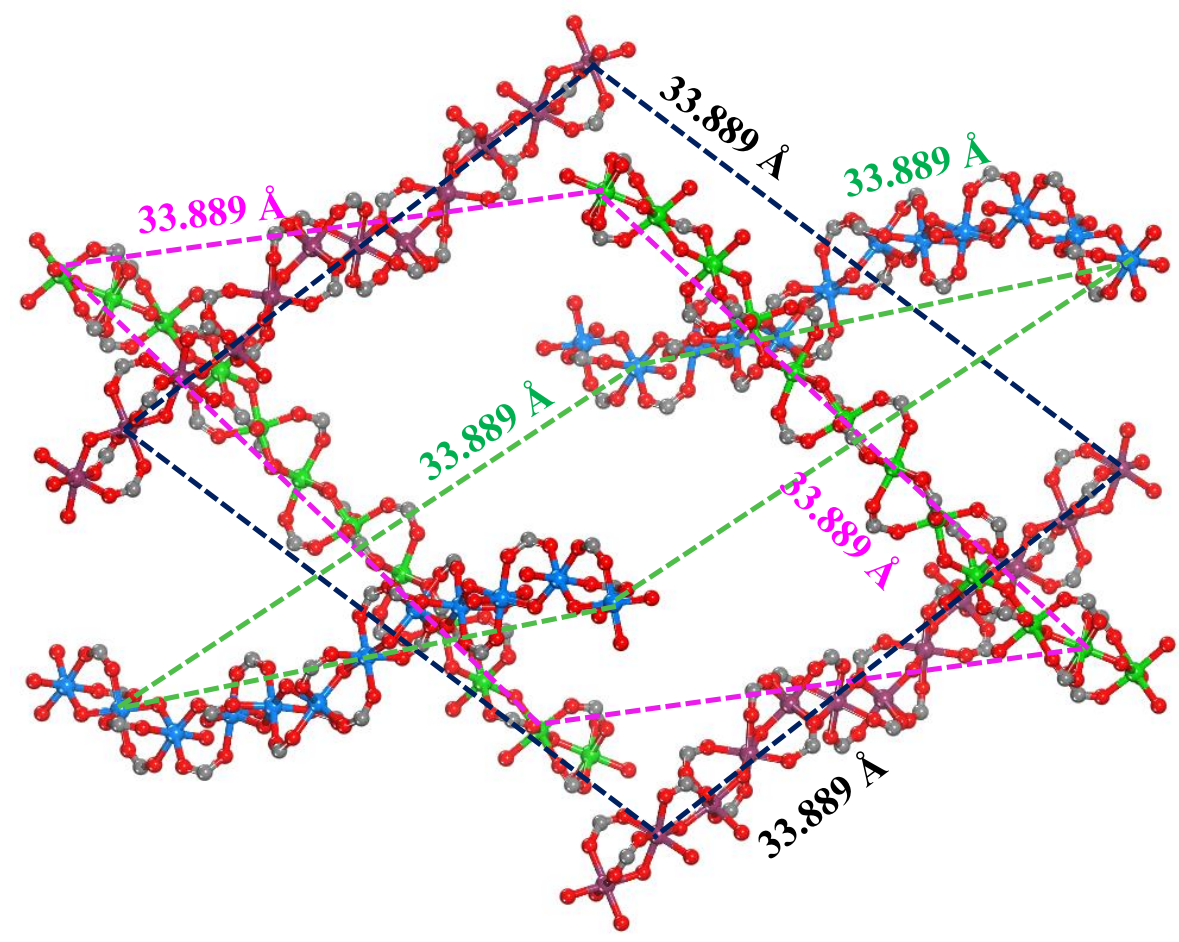

Figure S3. Schematic representation showing that spacing along the rods (translation) and between the rods in $3 \mathrm{~W}-\mathrm{ROD}-1$ is $33.889 \AA$. 
(a)
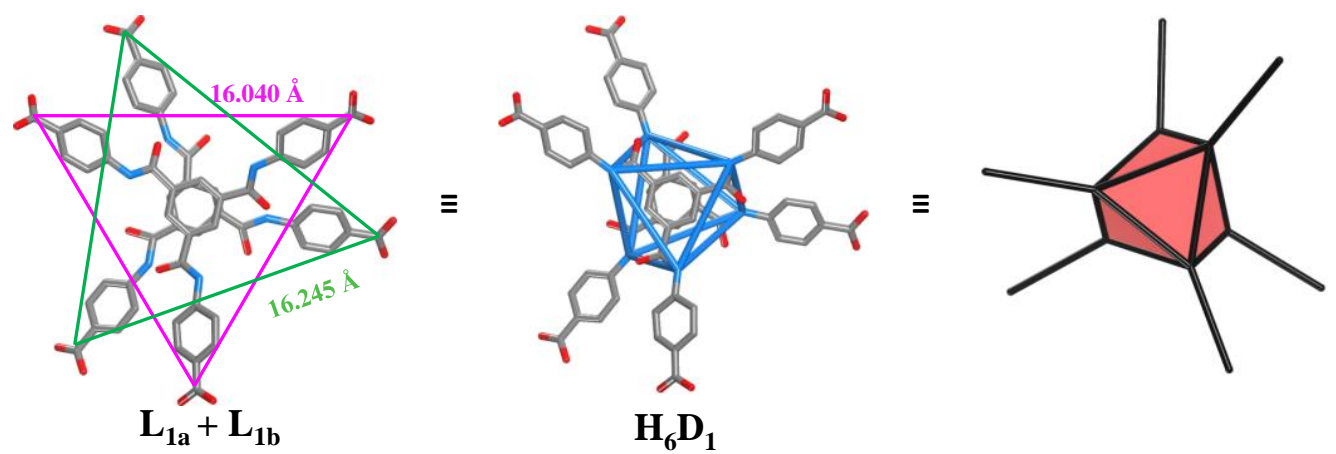

(b)

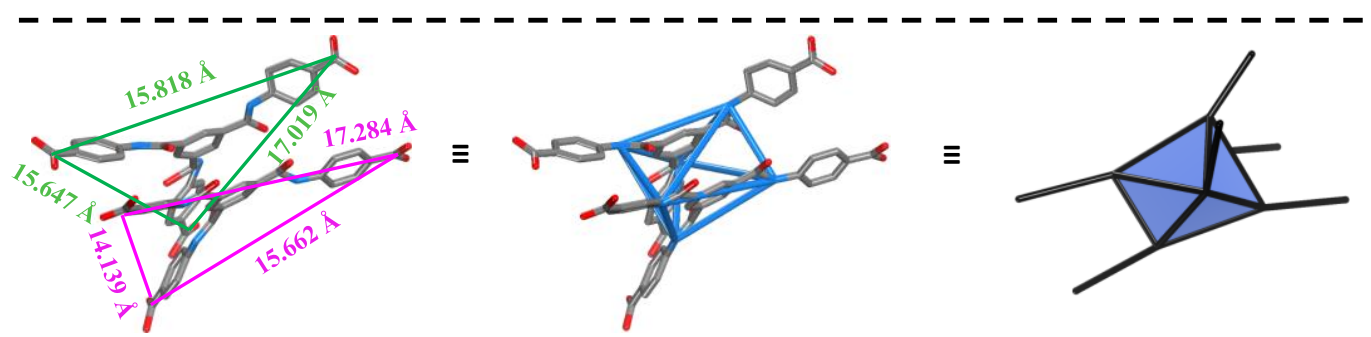

$\mathbf{L}_{1 \mathrm{c}}+\mathbf{L}_{1 \mathrm{~d}}$

\section{$\mathrm{H}_{6} \mathrm{D}_{2}$}

(c)
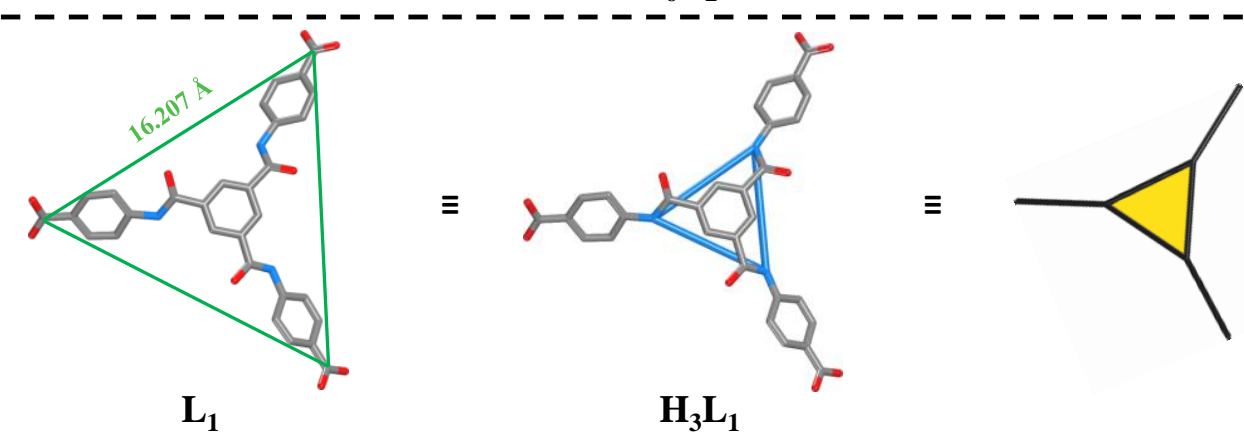

Figure S4. Two kinds of pseudo hexacarboxylic acids formed by two pairs of slightly different dimers of $\left[\mathrm{L}_{1} \cdots \cdots \mathrm{L}_{1 b}\right]$ (i.e., $\mathrm{H}_{6} \mathrm{D}_{1}$ ) (a) associated with the red octahedron SBU and $\left[\mathrm{L}_{1} \cdots \mathrm{L}_{1 d}\right]$ (i.e., $\mathrm{H}_{6} \mathrm{D}_{2}$ ) (b) associated with the blue octahedron and one single $\mathrm{H}_{3} \mathrm{~L}_{1}$ (c) associated with the yellow triangle in $3 \mathrm{~W}-\mathrm{ROD}-1 . \mathrm{O}=$ red, $\mathrm{N}=$ blue, and $\mathrm{C}=$ gray. 
(a)

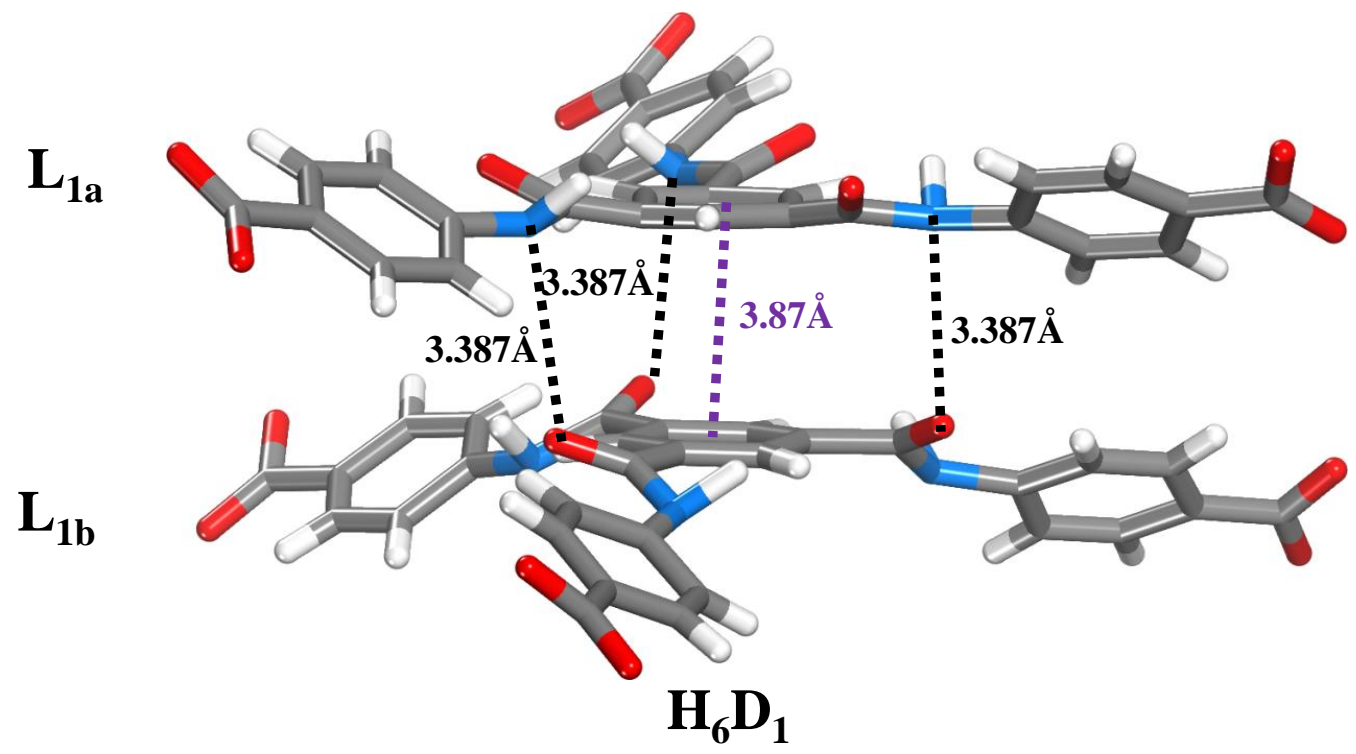

(b)

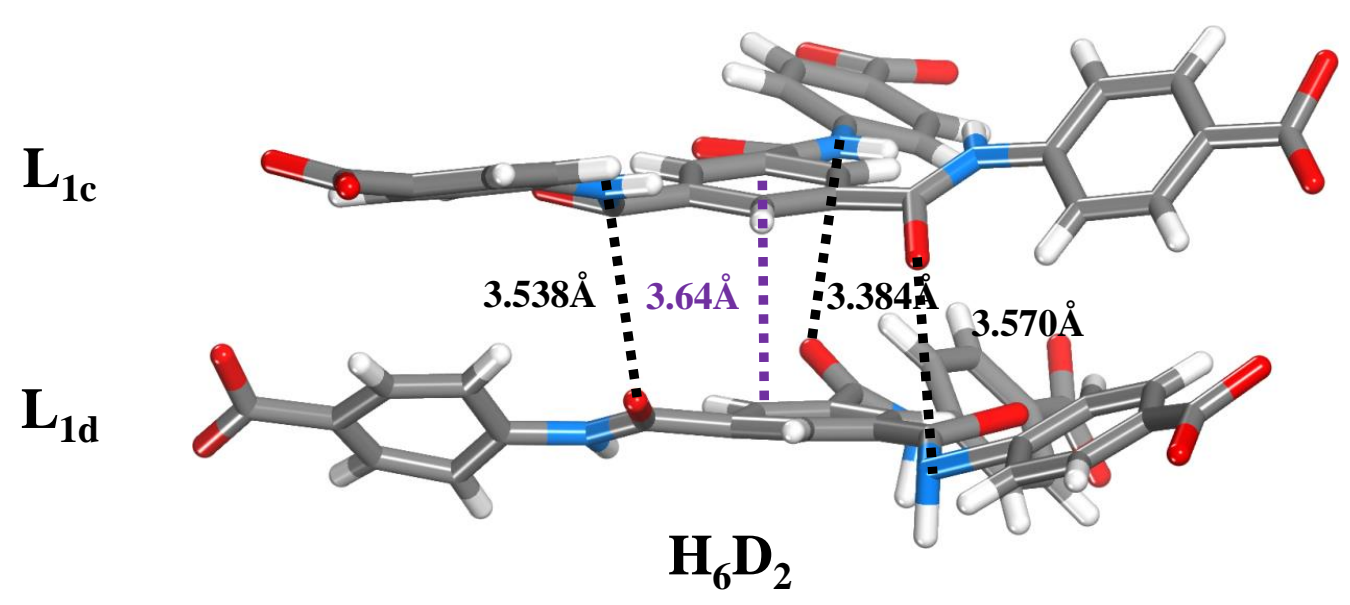

Figure S5. Two kinds of pseudo hexacarboxylic acids formed by two pairs of slightly different dimers of $\left[\mathrm{L}_{1} \cdots \cdots \mathrm{L}_{1 b}\right]$ (i.e., $\mathrm{H}_{6} \mathrm{D}_{1}$ ) (a) and $\left[\mathrm{L}_{1} c^{\cdots} \mathrm{L}_{1 d}\right]$ (i.e., $\mathrm{H}_{6} \mathrm{D}_{2}$ ) (b), respectively, through $\pi-\pi$ stacking interactions (i.e., $3.64 \AA$ and $3.87 \AA$ ) as well as hydrogen bonds (ranging from 3.384 to $3.570 \AA$ ) in $3 \mathrm{~W}-\mathrm{ROD}-1$. The black dotted line represents hydrogen bonds, distance between $\mathrm{N}$ atom to $\mathrm{O}$ atom and the purple dotted line represents $\pi-\pi$ stacking interactions between the neighboring two phenyl rings. $\mathrm{O}$ $=$ red, $\mathrm{N}=$ blue, $\mathrm{C}=$ gray and $\mathrm{H}=$ white. 
(a)

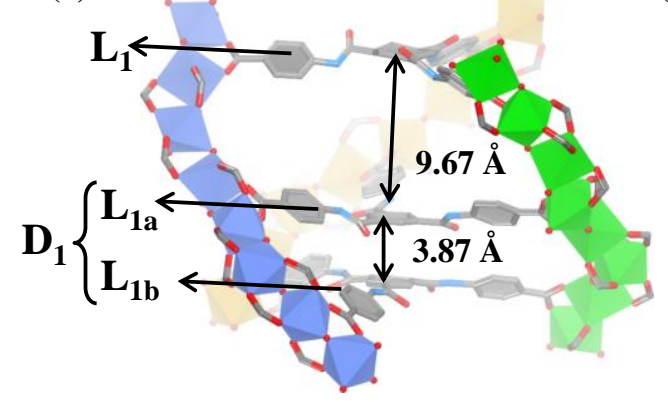

(c)

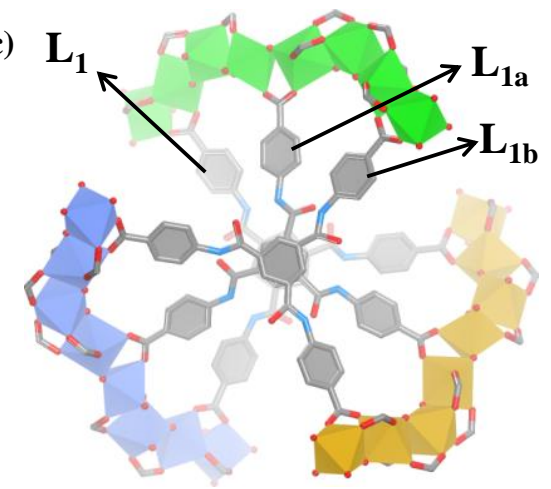

(b)

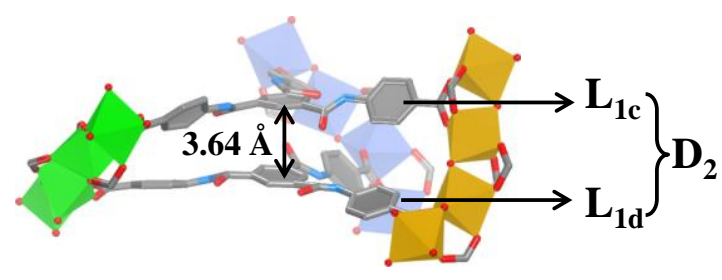

(d)

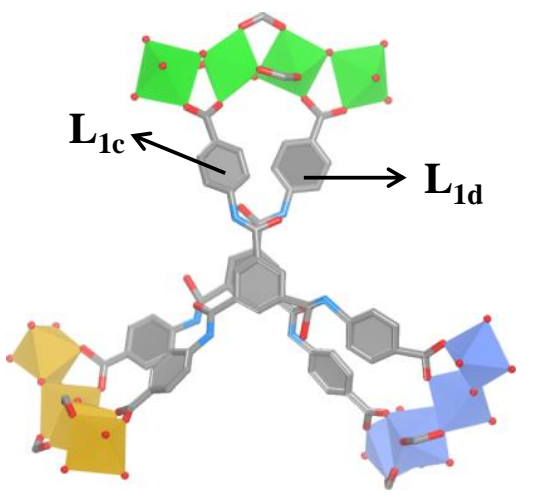

Figure S6. Schematic representation showing the distance between $\mathrm{L}_{1}$ and $\mathrm{L}_{1 \mathrm{a}}(\mathrm{a})$, and the connections between three-way rod MBBs and $\mathrm{D}_{1}$ together with $\mathrm{L}_{1}(\mathrm{a})$, and $\mathrm{D}_{2}$ (b) within $3 \mathrm{~W}$-ROD-1, as well as their respective views from $c$-axis (c) and [1-12] direction $(\mathrm{d})$. 


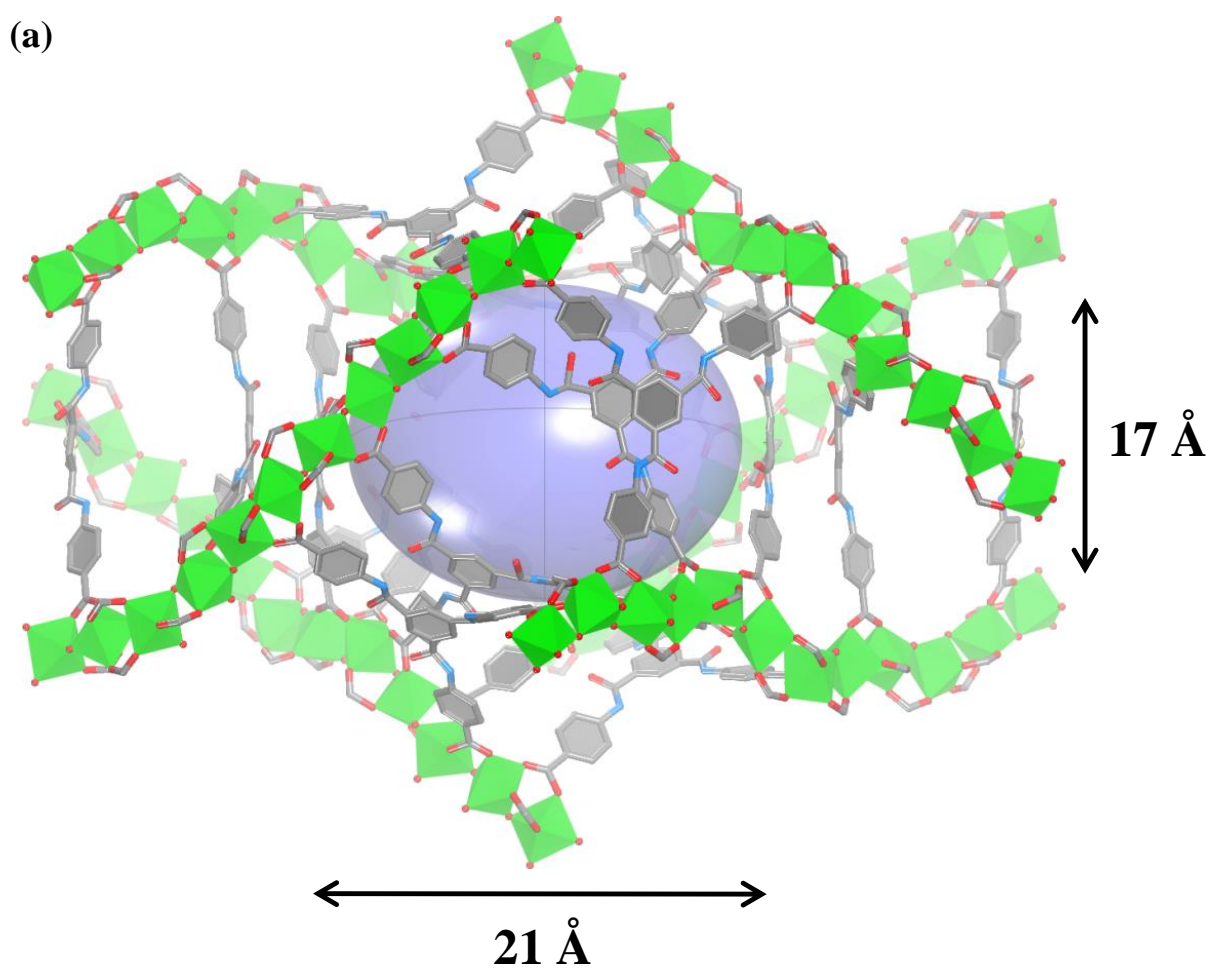

(b)

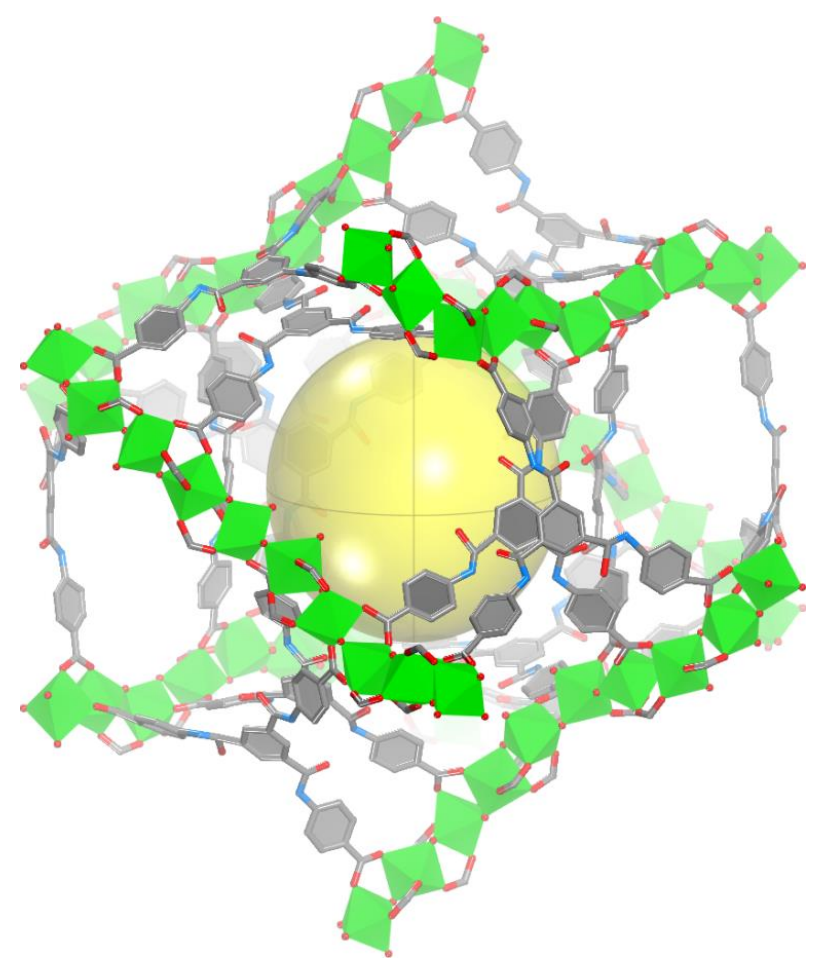

$16 \times 22 \AA^{2}$

Figure S7. Two types of cages within $3 \mathrm{~W}-\mathrm{ROD}-1$. In $=$ green, $\mathrm{O}=\mathrm{red}, \mathrm{N}=$ blue, and $\mathrm{C}=$ gray. 


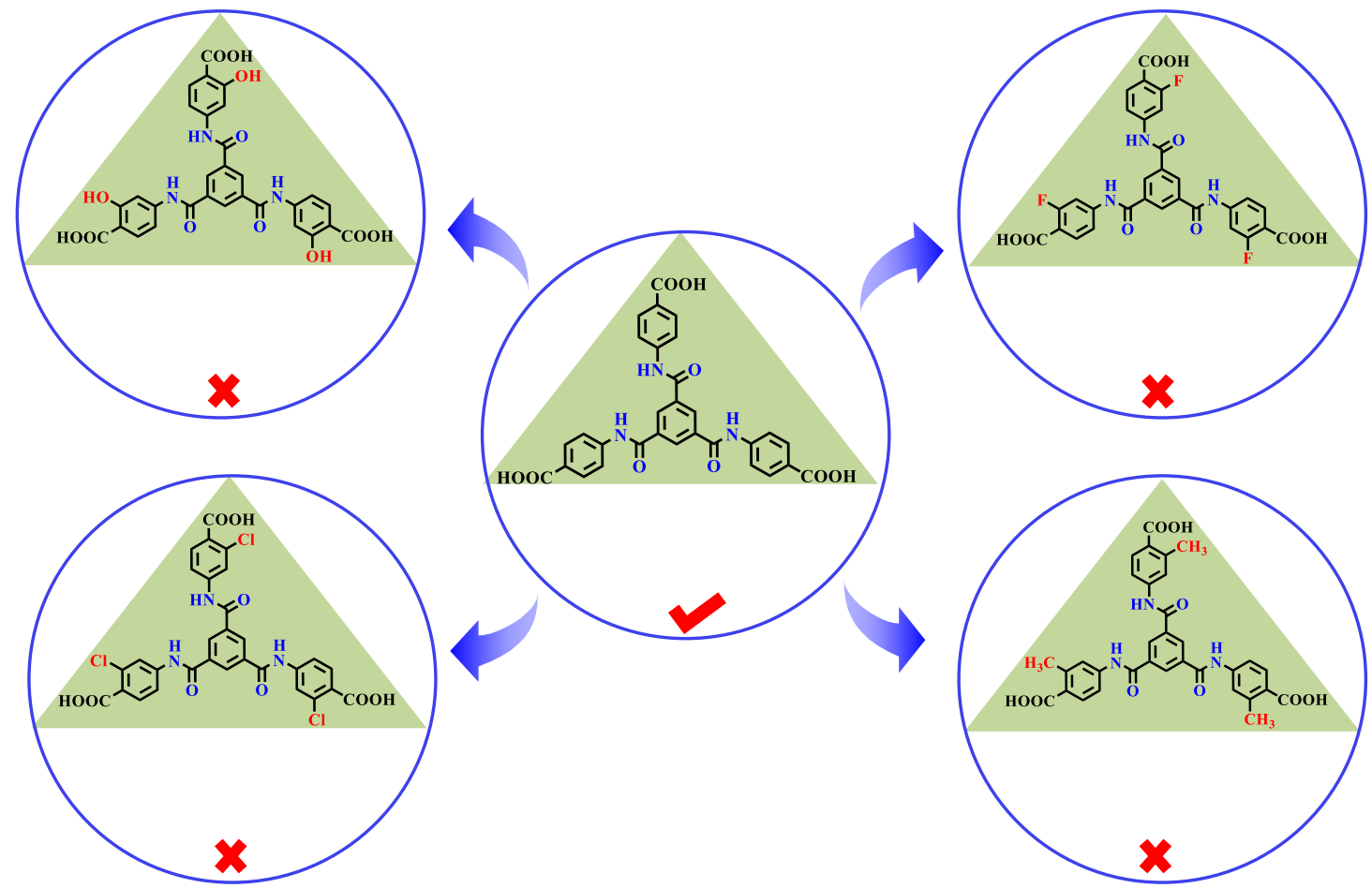

Figure S8. Schematic representation showing the appreciable tritopic $\mathrm{H}_{3} \mathrm{~L}_{1}$ rather than $\mathrm{H}_{3} \mathrm{~L}_{1}-(\mathrm{OH})_{3}, \mathrm{H}_{3} \mathrm{~L}_{1}-\mathrm{F}_{3}, \mathrm{H}_{3} \mathrm{~L}_{1}-(\mathrm{Cl})_{3}$ and $\mathrm{H}_{3} \mathrm{~L}_{1}-\left(\mathrm{CH}_{3}\right)_{3}$ to isolate the intricate $3 \mathrm{~W}-\mathrm{ROD}-1$.

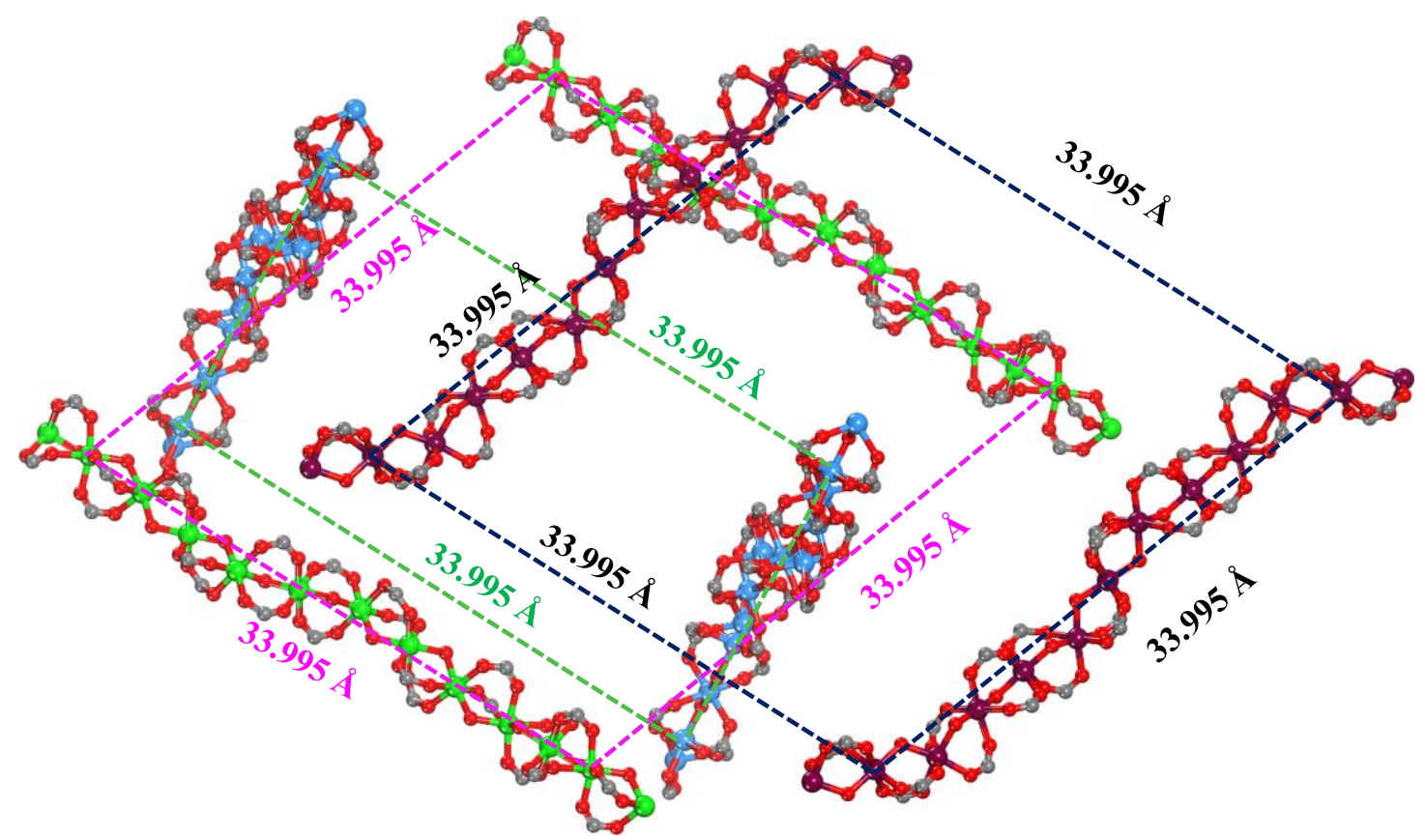

Figure S9 Schematic representation showing that spacing along the rods (translation) and between the rods in $3 \mathrm{~W}-\mathrm{ROD}-2-\mathrm{OH}$ is $33.995 \AA$. 
(a)

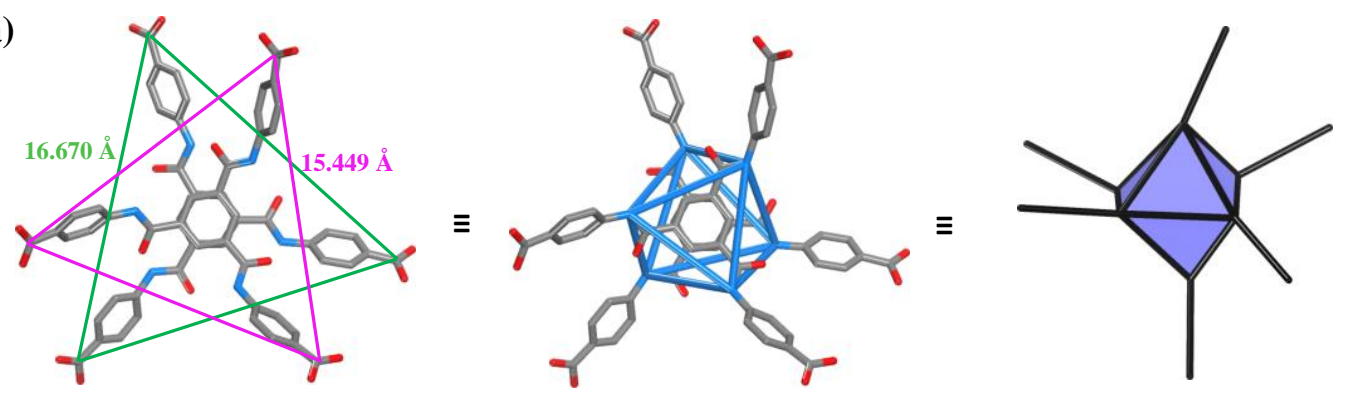

$\mathbf{L}_{1 \mathrm{e}}+\mathbf{L}_{1 \mathrm{f}}$

$\mathbf{H}_{6} \mathbf{D}_{3}$

(b)

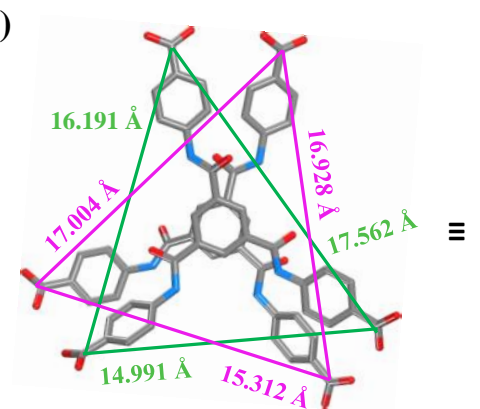

$L_{1 g}+L_{1 h}$

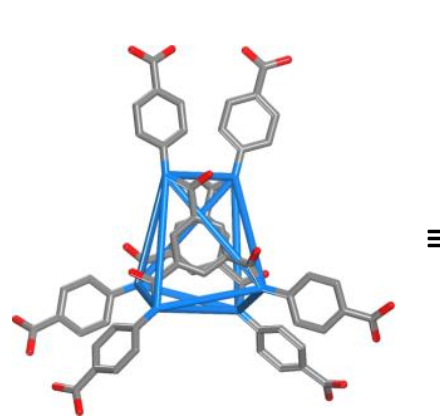

$\mathbf{H}_{6} \mathbf{D}_{4}$

Figure S10. Two kinds of pseudo hexacarboxylic acids formed by two pairs of slightly different dimers of $\left[\mathrm{L}_{1 \mathrm{e}}{ }^{\cdots} \mathrm{L}_{1 f}\right]$ (i.e., $\mathrm{H}_{6} \mathrm{D}_{3}$ ) (a) associated with the blue octahedron SBU and $\left[\mathrm{L}_{1 \mathrm{~g}} \cdots \mathrm{L}_{1 \mathrm{~h}}\right]$ (i.e., $\mathrm{H}_{6} \mathrm{D}_{4}$ ) (b) associated with the red octahedron in $3 \mathrm{~W}-\mathrm{ROD}-2-\mathrm{OH}$. $\mathrm{O}=$ red, $\mathrm{N}=$ blue and $\mathrm{C}=$ gray. 
(a)

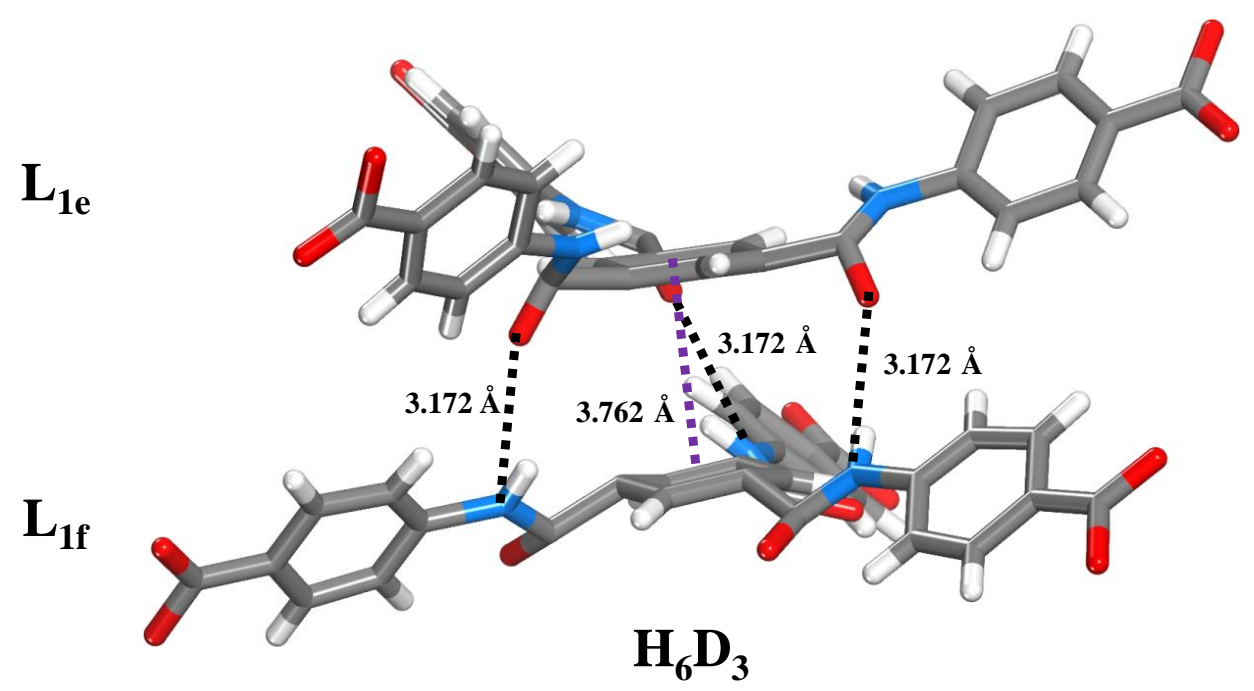

(b)

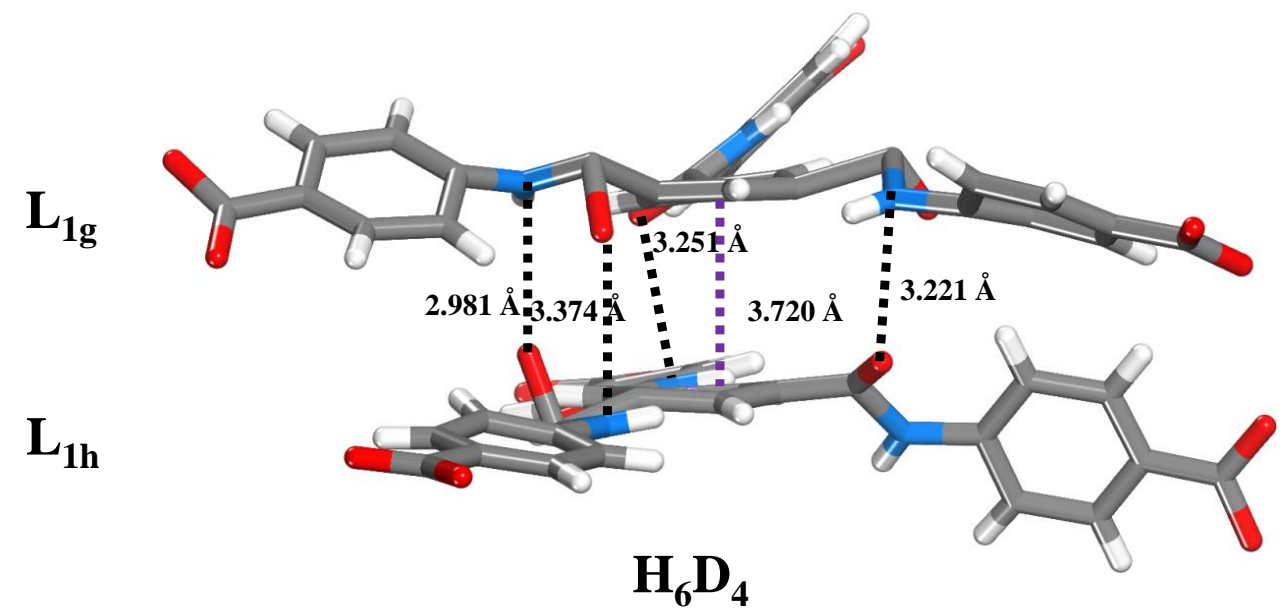

Figure S11. Two kinds of pseudo hexacarboxylic acids formed by two pairs of slightly different dimers of $\left[\mathrm{L}_{1}{ }^{\cdots} \mathrm{L}_{1 f}\right]$ (i.e., $\mathrm{H}_{6} \mathrm{D}_{3}$ ) (a) and $\left[\mathrm{L}_{1}{ }^{\cdots} \mathrm{L}_{1 h}\right]$ (i.e., $\mathrm{H}_{6} \mathrm{D}_{4}$ ) (b), respectively, through $\pi-\pi$ stacking interactions (3.720 $\mathrm{A}, 3.762 \AA$ ) as well as hydrogen bonds (ranging from 3.384 to $3.570 \AA$ ) in $3 \mathrm{~W}-\mathrm{ROD}-2-\mathrm{OH}$. The black dotted line represents hydrogen bonds, distance between $\mathrm{N}$ atom to $\mathrm{O}$ atom and the purple dotted line represents $\pi-\pi$ stacking interactions between the neighboring two phenyl rings. $\mathrm{O}=$ red, $\mathrm{N}=$ blue, $\mathrm{C}=$ gray and $\mathrm{H}=$ white. 
(a)

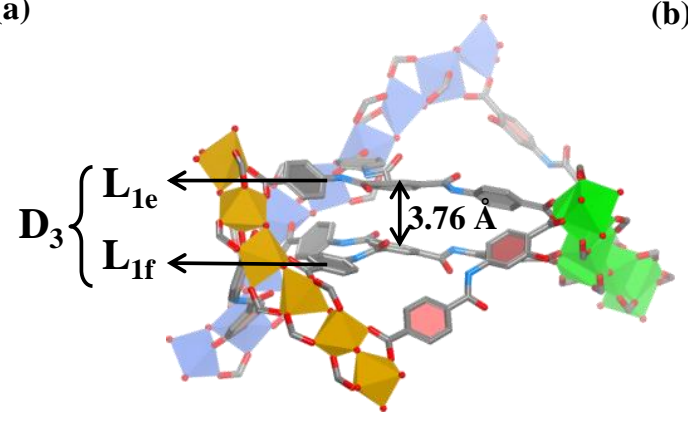

(c)

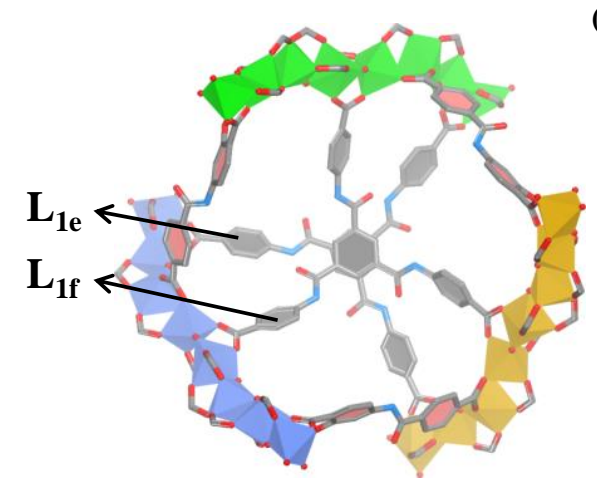

(b)

(d)
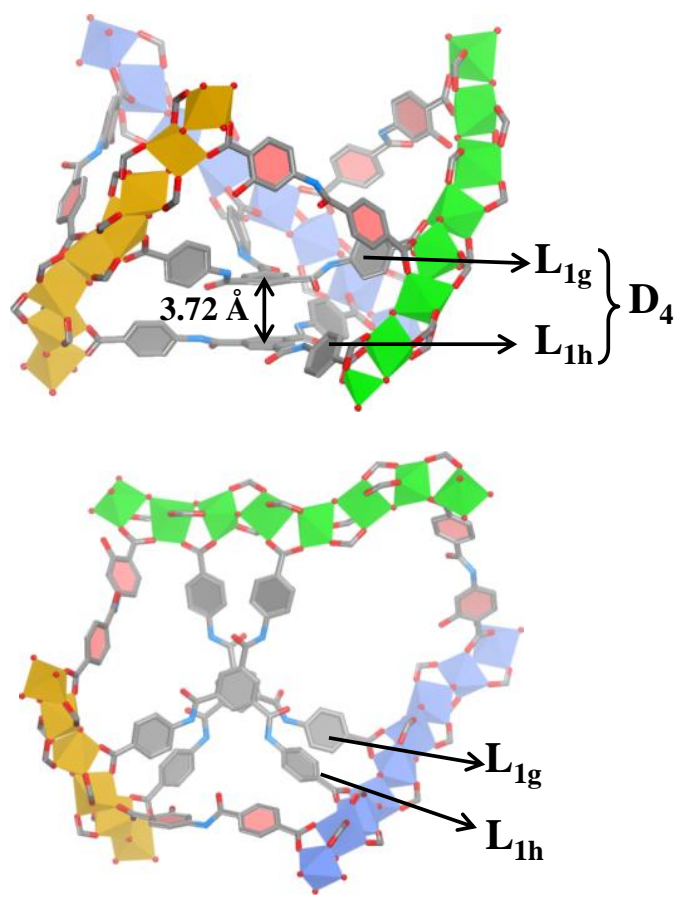

Figure S12. Schematic representation showing the connections between the threeway rod MBBs and $\mathrm{D}_{3}$ together with $\mathrm{L}_{2}-\mathrm{OH}(\mathrm{a})$, and $\mathrm{D}_{4}$ together with $\mathrm{L}_{2}-\mathrm{OH}$ (b) within $3 \mathrm{~W}-\mathrm{ROD}-2-\mathrm{OH}$, as well as their respective views along $c$-axis (c) and [1-12] direction (d). 


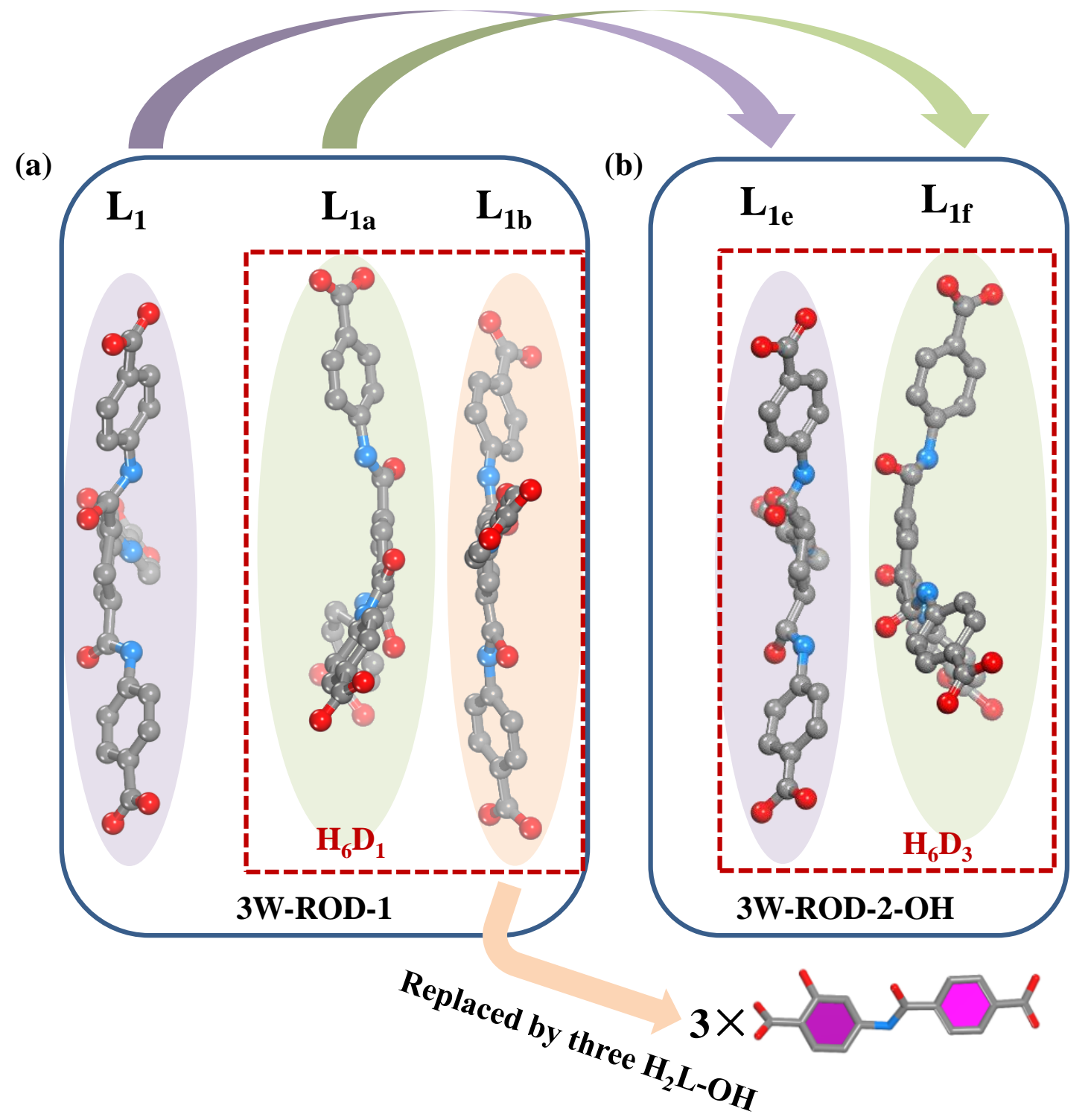

Figure S13. Schematic representation showing the ligand relocations between $3 \mathrm{~W}$ ROD-1 and 3W-ROD-2-OH along $c$-axis. 
(a)

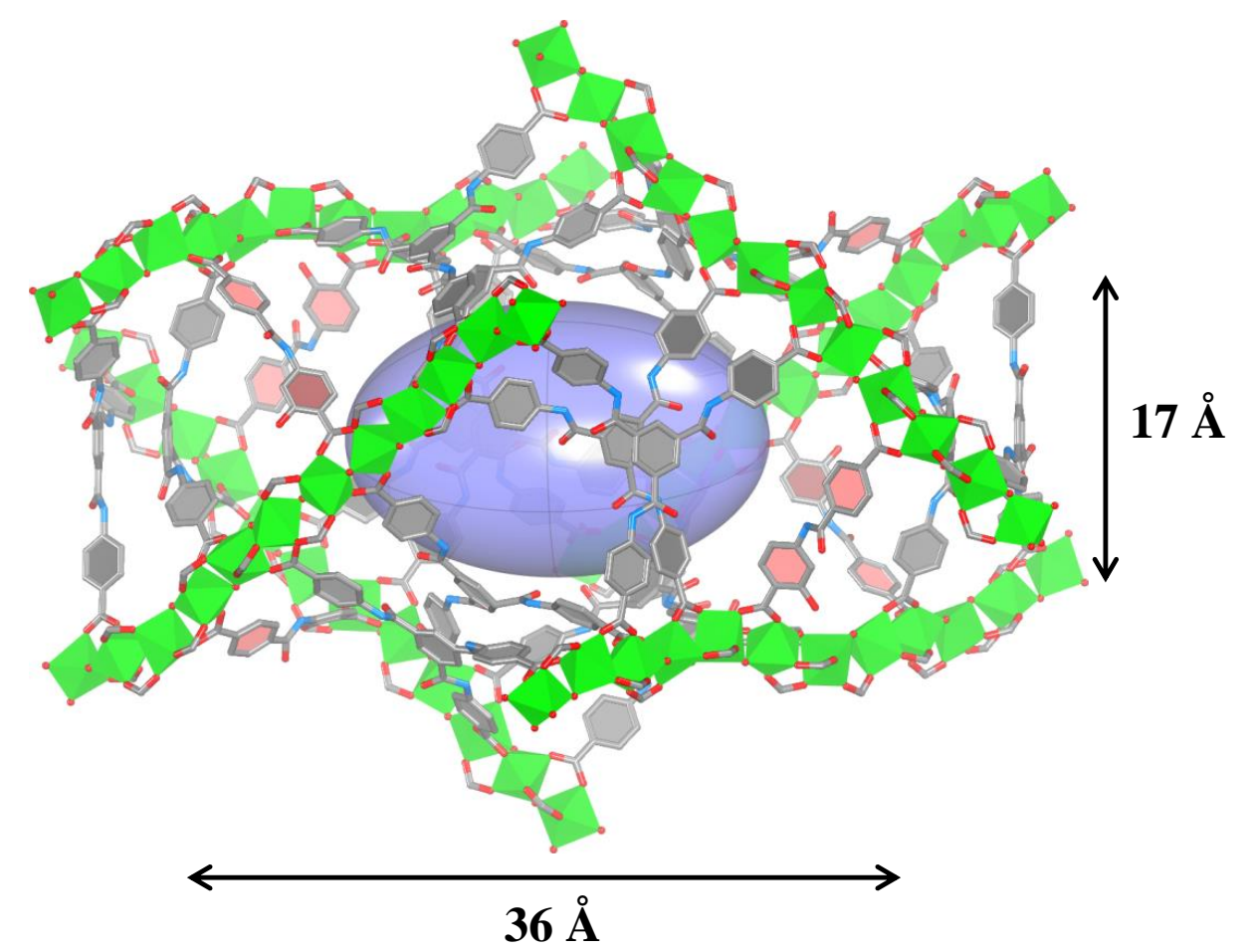

(b)

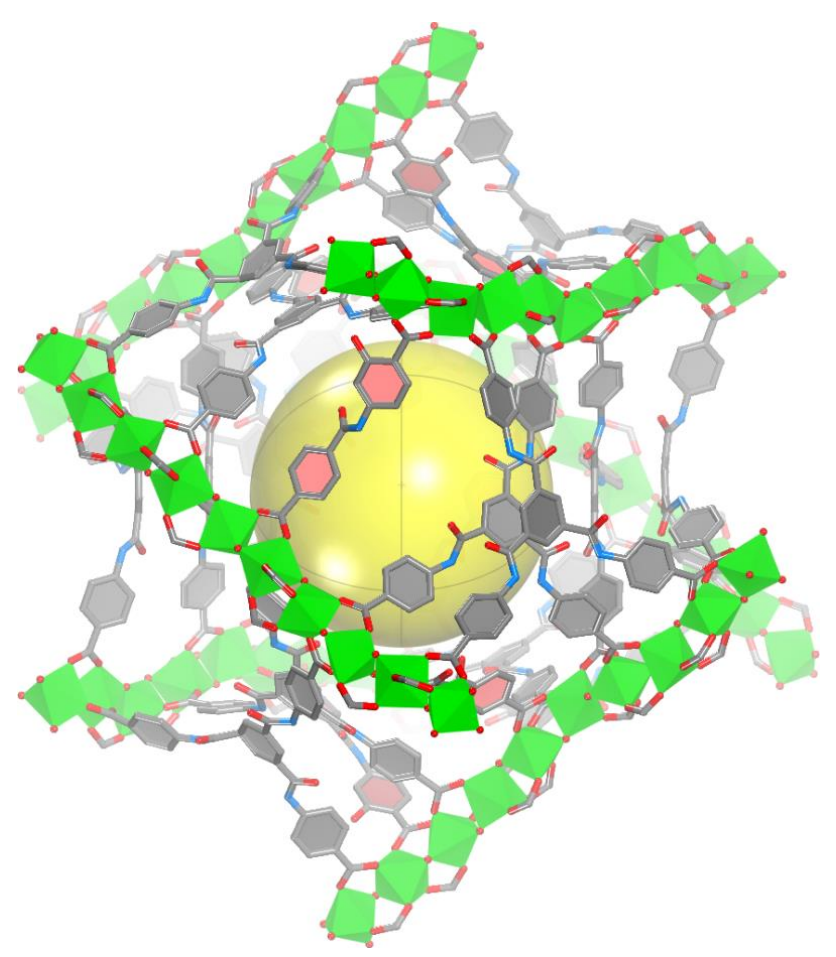

\section{$18 \times 20 \AA^{2}$}

Figure S14. Two types of cages within $3 \mathrm{~W}-\mathrm{ROD}-2-\mathrm{OH}$. In $=$ green, $\mathrm{O}=$ red, $\mathrm{N}=$ blue, and $\mathrm{C}=$ gray. 


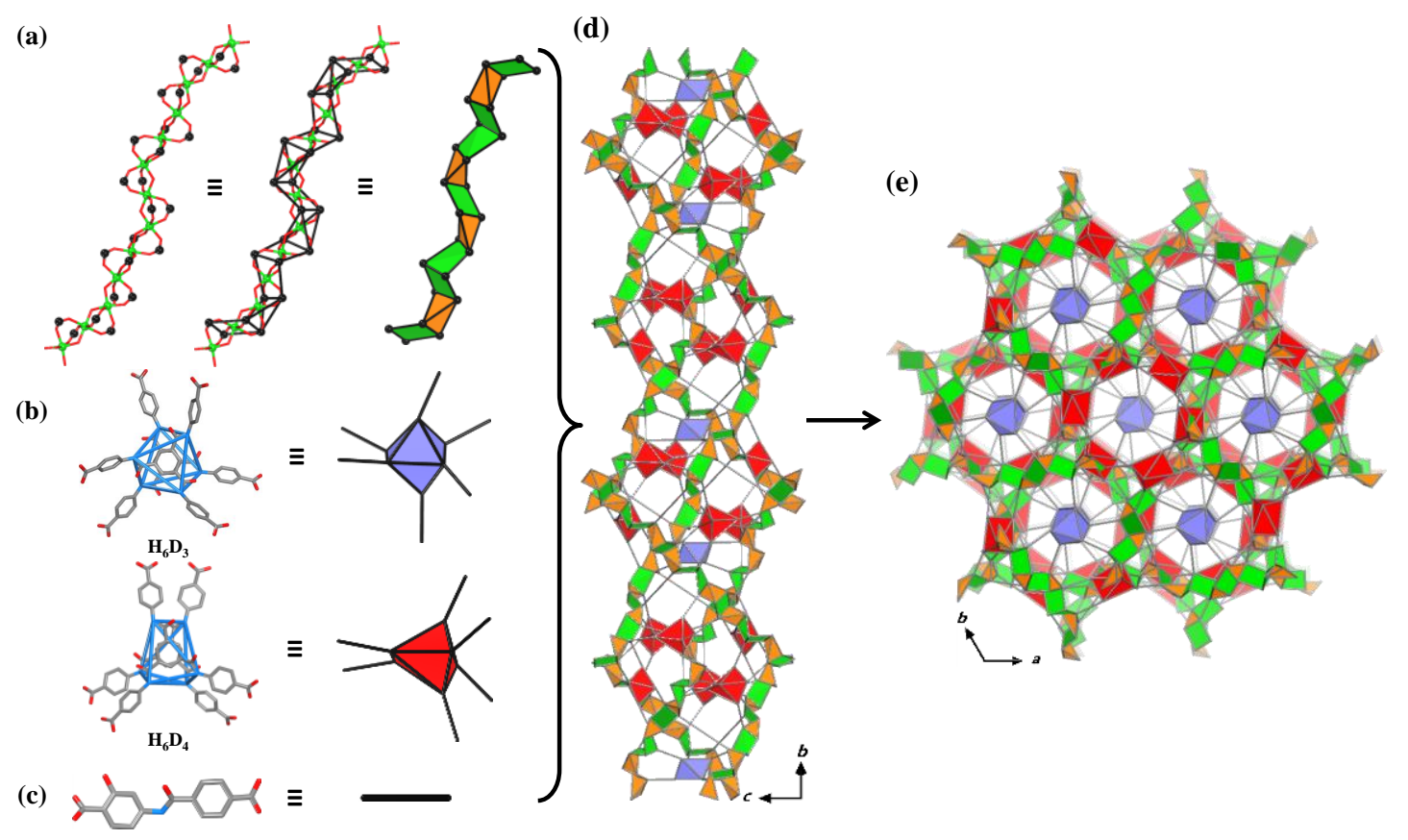

Figure S15. Deconstruction of $3 \mathrm{~W}-\mathrm{ROD}-2-\mathrm{OH}$ for topological analysis. (a) Simplifying the lengthy MBB of $\left[\operatorname{In}_{11}(\mathrm{OH})_{11}\left(\mathrm{O}_{2} \mathrm{C}-\right)_{22}\right]_{\infty}$ into a pair of opposite edgesharing rectangle (green), tetrahedron (orange), two quadrangles (green) and another tetrahedron with one more centered rectangle. (b) Regarding the pseudo hexacarboxylates of $\mathrm{D}_{3}{ }^{6-}$ and $\mathrm{D}_{4}{ }^{6-}$ as distorted octahedral SBUs. (c) Regarding the $\mathrm{L}_{2-}$ $\mathrm{OH}^{2-}$ as linear SBU. (d) Topological views along $a$-axis and (e) $c$-axis, respectively. 
Systre Output File

\section{CRYSTAL \\ NAME 3W-ROD-1}

\section{GROUP $R-3$}

CELL 7.08390 7.08390 12.8961090.000090.0000120.0000

NODE 1: $5 \quad 0.341880 .010540 .15589$

NODE 2: $5 \quad 0.091590 .077930 .23315$

NODE 3: $4 \quad 0.326230 .062970 .03942$

NODE 4: $5 \quad 0.133550 .217740 .09040$

NODE 5: $5 \quad 0.207310 .234630 .02366$

NODE 6: $5 \quad 0.054930 .387270 .07215$

NODE 7: $5 \quad 0.311590 .137350 .13424$

NODE 8: $5 \quad 0.301750 .369190 .05654$

NODE 9: $4 \quad 0.092350 .234270 .36350$

NODE 10: $5 \quad 0.241950 .356220 .12290$

NODE 11: $5 \quad 0.240880 .038930 .18523$

NODE 12: $5 \quad 0.009180 .087370 .17084$

NODE 13: $5 \quad 0.004540 .237750 .10500$

NODE 14: $4 \quad 0.238520 .227550 .23576$

NODE 15: 50.170410 .351720 .05067

NODE 16: 30.064320 .091700 .33469

NODE 17: $5 \quad 0.274840 .238510 .09506$

NODE 18: $4 \quad 0.421710 .031270 .00863$

EDGE $0.207310 .23463 \quad 0.02366 \quad 0.274840 .238510 .09506$

EDGE $0.326230 .062970 .03942 \quad 0.33234-0.054930 .07215$

EDGE $0.170410 .351720 .05067 \quad 0.241950 .356220 .12290$

EDGE $0.004540 .237750 .10500 \quad-0.038930 .201950 .18523$

$\begin{array}{llllll}\text { EDGE } 0.20731 & 0.23463 & 0.02366 & 0.26326 & 0.32623 & -0.03942\end{array}$

EDGE $0.091590 .07793 \quad 0.23315-0.077930 .013650 .23315$

EDGE $0.241950 .35622 \quad 0.12290 \quad 0.301750 .369190 .05654$

EDGE $0.133550 .217740 .09040 \quad 0.170410 .351720 .05067$

EDGE $0.341880 .01054 \quad 0.155890 .42472-0.022880 .21043$

$\begin{array}{lllllll}\text { EDGE } 0.13355 & 0.21774 & 0.09040 & 0.20731 & 0.23463 & 0.02366\end{array}$

EDGE $0.009180 .087370 .17084-0.038930 .201950 .18523$

EDGE $0.00454 \quad 0.237750 .10500 \quad-0.137350 .174240 .13424$

EDGE $0.240880 .038930 .18523 \quad 0.311590 .137350 .13424$

EDGE $0.064320 .091700 .334690 .02737-0.064320 .33469$

EDGE $0.311590 .137350 .13424 \quad 0.341880 .010540 .15589$

EDGE $0.05493 \quad 0.38727 \quad 0.07215 \quad-0.105790 .322360 .09758$

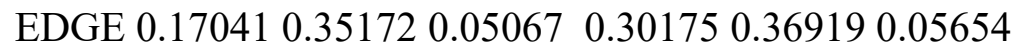

EDGE $0.064320 .091700 .33469 \quad 0.092350 .234270 .36350$

EDGE $0.274840 .23851 \quad 0.09506 \quad 0.241950 .356220 .12290$

EDGE $0.326230 .062970 .03942 \quad 0.428150 .105790 .09758$

EDGE $0.004540 .237750 .10500-0.062970 .263260 .03942$

EDGE $0.009180 .087370 .17084-0.077930 .013650 .23315$

EDGE $0.170410 .351720 .05067 \quad 0.054930 .387270 .07215$

EDGE $0.004540 .237750 .10500 \quad 0.133550 .217740 .09040$

EDGE $0.240880 .03893 \quad 0.18523 \quad 0.355080 .195980 .19909$

EDGE $0.054930 .387270 .07215-0.031270 .390440 .00863$ 
EDGE $0.421710 .031270 .00863 \quad 0.369190 .06744-0.05654$ EDGE $0.207310 .23463 \quad 0.02366 \quad 0.170410 .351720 .05067$ EDGE $0.421710 .031270 .00863 \quad 0.42568-0.099070 .03017$ EDGE $0.133550 .217740 .09040 \quad 0.241950 .356220 .12290$ EDGE $0.009180 .087370 .17084 \quad 0.091590 .077930 .23315$ EDGE $0.133550 .217740 .09040 \quad 0.274840 .238510 .09506$ EDGE $0.274840 .238510 .09506 \quad 0.301750 .369190 .05654$ EDGE 0.341880 .010540 .155890 .428150 .105790 .09758 EDGE $0.240880 .03893 \quad 0.18523 \quad 0.341880 .010540 .15589$ EDGE $0.009180 .087370 .17084 \quad 0.07819-0.009180 .17084$ $\begin{array}{llllll}\text { EDGE } 0.09159 & 0.07793 & 0.23315 & 0.23852 & 0.22755 & 0.23576\end{array}$ EDGE $0.311590 .137350 .13424 \quad 0.274840 .238510 .09506$ EDGE 0.00454 0.23775 0.10500 -0.010540 .331340 .15589$ EDGE $0.05493 \quad 0.387270 .07215 \quad 0.099070 .524750 .03017$ $\begin{array}{llllll}\text { EDGE } 0.42171 & 0.03127 & 0.00863 & 0.57432 & 0.09907 & -0.03017\end{array}$ EDGE $0.207310 .234630 .02366 \quad 0.301750 .369190 .05654$ \#EDGE_CENTER 0.24107 0.23657 0.05936 \#EDGE_CENTER 0.329280 .004020 .05578 \#EDGE_CENTER 0.20618 0.353970.08678 \#EDGE_CENTER -0.01719 0.219850.14512 \#EDGE_CENTER 0.23528 0.28043 -0.00788 \#EDGE CENTER 0.00683 0.04579 0.23315 \#EDGE CENTER 0.27185 0.36270 0.08972 \#EDGE_CENTER 0.151980 .284730 .07053 \#EDGE_CENTER 0.38330 -0.00617 0.18316 \#EDGE_CENTER 0.17043 0.22618 0.05703 \#EDGE_CENTER -0.01488 0.14466 0.17804 \#EDGE_CENTER -0.06640 0.205990.11962 \#EDGE_CENTER 0.276230 .088140 .15974 \#EDGE_CENTER 0.045850 .013690 .33469 \#EDGE_CENTER 0.326730 .073940 .14506 \#EDGE_CENTER -0.02543 0.354810.08486 \#EDGE_CENTER 0.23608 0.36045 0.05361 \#EDGE_CENTER 0.07834 0.16298 0.34910 \#EDGE_CENTER 0.25839 0.297360.10898 \#EDGE_CENTER 0.377190 .084380 .06850 \#EDGE_CENTER -0.02921 0.25050 0.07221 \#EDGE_CENTER -0.03438 0.05051 0.20199 \#EDGE_CENTER 0.11267 0.36950 0.06141 \#EDGE_CENTER 0.06905 0.227740.09770 \#EDGE_CENTER 0.29798 0.11745 0.19216 \#EDGE_CENTER 0.011830 .388850 .04039 \#EDGE_CENTER 0.39545 0.04935 -0.02396 \#EDGE_CENTER 0.18886 0.293170.03716 \#EDGE_CENTER $0.42370-0.033900 .01940$ \#EDGE_CENTER 0.18775 0.28698 0.10665 \#EDGE_CENTER 0.050380 .082650 .20199 \#EDGE_CENTER 0.20420 0.228120.09273 \#EDGE_CENTER 0.288300 .303850 .07580 \#EDGE_CENTER 0.385010 .058160 .12673 
\#EDGE CENTER 0.291380 .024730 .17056 \#EDGE_CENTER 0.043680 .039100 .17084 \#EDGE CENTER 0.165050 .152740 .23445 \#EDGE CENTER 0.293220 .187930 .11465 \#EDGE_CENTER - 0.003000 .284540 .13044 \#EDGE_CENTER 0.077000 .456010 .05116 \#EDGE_CENTER $0.498010 .06517-0.01077$ \#EDGE_CENTER 0.254530 .301910 .04010 END 
Systre Output File

\section{CRYSTAL \\ NAME 3W-ROD-2-OH}

\section{GROUP $R-3$}

CELL 7.39928 7.39928 12.5065990.000090.0000 120.0000

NODE 1: $4 \quad 0.063440 .335410 .22116$

NODE 2: $5 \quad 0.306840 .002760 .29554$

NODE 3: $5 \quad 0.221960 .086110 .41384$

NODE 4: $5 \quad 0.250450 .279080 .12098$

NODE 5: $5 \quad 0.087760 .062380 .17017$

NODE 6: $5 \quad 0.126020 .299020 .10108$

NODE 7: $5 \quad 0.105160 .399360 .14780$

NODE 8: $4 \quad 0.024880 .207410 .26139$

NODE 9: $5 \quad 0.313660 .082280 .16943$

NODE 10: $5 \quad 0.072870 .460340 .08353$

NODE 11: $5 \quad 0.221640 .220380 .38647$

NODE 12: $5 \quad 0.157270 .351380 .29832$

NODE 13: $5 \quad 0.319330 .008550 .10363$

NODE 14: $5 \quad 0.027570 .087800 .23486$

NODE 15: 50.415860 .041530 .02215

NODE 16: $5 \quad 0.224270 .134500 .13244$

NODE 17: $5 \quad 0.071230 .347110 .03769$

NODE 18: $5 \quad 0.324660 .100440 .24860$

EDGE $0.379730 .120360 .07472 \quad 0.342010 .232890 .08474$

$\begin{array}{lllllll}\text { EDGE } 0.31933 & 0.00855 & 0.10363 & 0.27588 & -0.07123 & 0.03769\end{array}$

EDGE $0.126020 .299020 .10108 \quad 0.071230 .347110 .03769$

EDGE $0.415860 .04153 \quad 0.02215 \quad 0.490600 .01804-0.03501$

EDGE $0.063440 .335410 .22116 \quad 0.111690 .446290 .28020$

EDGE $0.319330 .008550 .10363 \quad 0.38747-0.072870 .08353$

EDGE $0.087760 .062380 .17017 \quad 0.027570 .087800 .23486$

EDGE $0.02488 \quad 0.207410 .26139-0.002760 .304080 .29554$

EDGE $0.224270 .134500 .13244 \quad 0.313660 .082280 .16943$

EDGE $0.319330 .008550 .10363 \quad 0.313660 .082280 .16943$

$\begin{array}{llllll}\text { EDGE } 0.08776 & 0.06238 & 0.17017 & 0.22427 & 0.13450 & 0.13244\end{array}$

$\begin{array}{lllllll}\text { EDGE } 0.41586 & 0.04153 & 0.02215 & 0.44502 & 0.11295 & -0.05314\end{array}$

EDGE $0.126020 .299020 .10108 \quad 0.197480 .444710 .08050$

EDGE $0.415860 .041530 .02215 \quad 0.50940-0.018040 .03501$

$\begin{array}{lllllll}\text { EDGE } 0.02488 & 0.20741 & 0.26139 & -0.10044 & 0.22421 & 0.24860\end{array}$

EDGE $0.072870 .460340 .08353 \quad 0.018040 .527440 .03501$

EDGE $0.319330 .008550 .10363 \quad 0.17300-0.126020 .10108$

EDGE $0.22427 \quad 0.13450 \quad 0.13244 \quad 0.250450 .279080 .12098$

EDGE 0.071230 .347110 .037690 .197480 .444710 .08050

EDGE $0.087760 .062380 .17017 \quad-0.062380 .025380 .17017$

EDGE $0.071230 .347110 .03769 \quad 0.072870 .460340 .08353$

$\begin{array}{llllll}\text { EDGE } 0.31933 & 0.00855 & 0.10363 & 0.29420 & -0.10516 & 0.14780\end{array}$

$\begin{array}{lllllll}\text { EDGE } 0.15727 & 0.35138 & 0.29832 & 0.11169 & 0.44629 & 0.28020\end{array}$

EDGE $0.105160 .399360 .14780 \quad 0.197480 .444710 .08050$

EDGE $0.313660 .082280 .16943 \quad 0.416220 .054250 .21235$

EDGE $0.306840 .002760 .29554 \quad 0.33460-0.111690 .28020$ 
EDGE $0.250450 .27908 \quad 0.12098 \quad 0.342010 .232890 .08474$ EDGE $0.072870 .460340 .08353 \quad 0.197480 .444710 .08050$ EDGE $0.22427 \quad 0.13450 \quad 0.13244 \quad 0.342010 .232890 .08474$ EDGE $0.126020 .299020 .10108 \quad 0.105160 .399360 .14780$ EDGE $0.313660 .082280 .16943 \quad 0.324660 .100440 .24860$ EDGE $0.087760 .062380 .17017 \quad 0.06022-0.027570 .23486$ EDGE $0.306840 .002760 .29554 \quad 0.19410-0.157270 .29832$ $\begin{array}{llllll}\text { EDGE } 0.07123 & 0.34711 & 0.03769 & 0.02925 & 0.35982 & -0.03780\end{array}$ EDGE $0.224270 .13450 \quad 0.13244 \quad 0.353010 .251050 .16390$ EDGE $0.415860 .041530 .02215 \quad 0.33057-0.02925-0.03780$ EDGE $0.250450 .27908 \quad 0.12098 \quad 0.394700 .396780 .11217$ EDGE $0.02757 \quad 0.08780 \quad 0.23486 \quad 0.06022 \quad-0.027570 .23486$ EDGE 0.379730 .120360 .074720 .415860 .041530 .02215 EDGE $0.063440 .33541 \quad 0.22116 \quad 0.105160 .399360 .14780$ EDGE $0.126020 .299020 .10108 \quad 0.250450 .279080 .12098$ $\begin{array}{lllllll}\text { EDGE } 0.10516 & 0.39936 & 0.14780 & 0.07287 & 0.46034 & 0.08353\end{array}$ EDGE $0.027570 .08780 \quad 0.23486 \quad 0.063440 .335410 .22116$ EDGE $0.221960 .08611 \quad 0.41384 \quad 0.221640 .220380 .38647$ \#EDGE_CENTER 0.360870 .176630 .07973 \#EDGE_CENTER 0.29761 -0.031340.07066 \#EDGE_CENTER 0.098620 .323070 .06939 \#EDGE_CENTER 0.453230 .02979 -0.00643 \#EDGE CENTER 0.08757 0.39085 0.25068 \#EDGE_CENTER $0.35340-0.032160 .09358$ \#EDGE_CENTER 0.057670 .075090 .20251 \#EDGE_CENTER 0.011060 .255740 .27846 \#EDGE_CENTER 0.268960.108390.15093 \#EDGE_CENTER 0.316490 .045420 .13653 \#EDGE_CENTER 0.15601 0.09844 0.15130 \#EDGE_CENTER 0.43044 0.07724 -0.01549 \#EDGE_CENTER 0.16175 0.371860.09079 \#EDGE_CENTER 0.462630 .011740 .02858 \#EDGE_CENTER -0.03778 0.215810.25499 \#EDGE CENTER 0.04546 0.493890.05927 \#EDGE_CENTER 0.24617 -0.05873 0.10236 \#EDGE_CENTER 0.23736 0.20679 0.12671 \#EDGE_CENTER 0.134360.395910.05910 \#EDGE_CENTER 0.01269 0.043880.17017 \#EDGE_CENTER 0.072050 .403730 .06061 \#EDGE_CENTER 0.30676 -0.04830 0.12572 \#EDGE_CENTER 0.13448 0.39883 0.28926 \#EDGE_CENTER 0.151320 .422030 .11415 \#EDGE_CENTER 0.364940 .068270 .19089 \#EDGE_CENTER 0.32072 -0.05446 0.28787 \#EDGE_CENTER 0.29623 0.255990.10286 \#EDGE_CENTER 0.135180 .452520 .08202 \#EDGE_CENTER 0.283140.18370 0.10859 \#EDGE_CENTER 0.115590.34919 0.12444 \#EDGE_CENTER 0.319160.091360.20901 \#EDGE_CENTER 0.073990 .017400 .20251 
\#EDGE CENTER 0.25047 -0.07725 0.29693

\#EDGE_CENTER 0.05024 0.35347 -0.00005

\#EDGE CENTER 0.28864 0.19278 0.14817

\#EDGE_CENTER 0.373210 .00614 -0.00782

\#EDGE_CENTER 0.322570 .337930 .11658

\#EDGE_CENTER 0.043900 .030110 .23486

\#EDGE CENTER 0.39779 0.080950.04844

\#EDGE_CENTER 0.084300 .367390 .18448

\#EDGE_CENTER 0.18823 0.28905 0.11103

\#EDGE CENTER 0.08902 0.429850.11567

\#EDGE_CENTER 0.024880 .207410 .26139

\#EDGE_CENTER 0.22180 0.153250.40015

END 


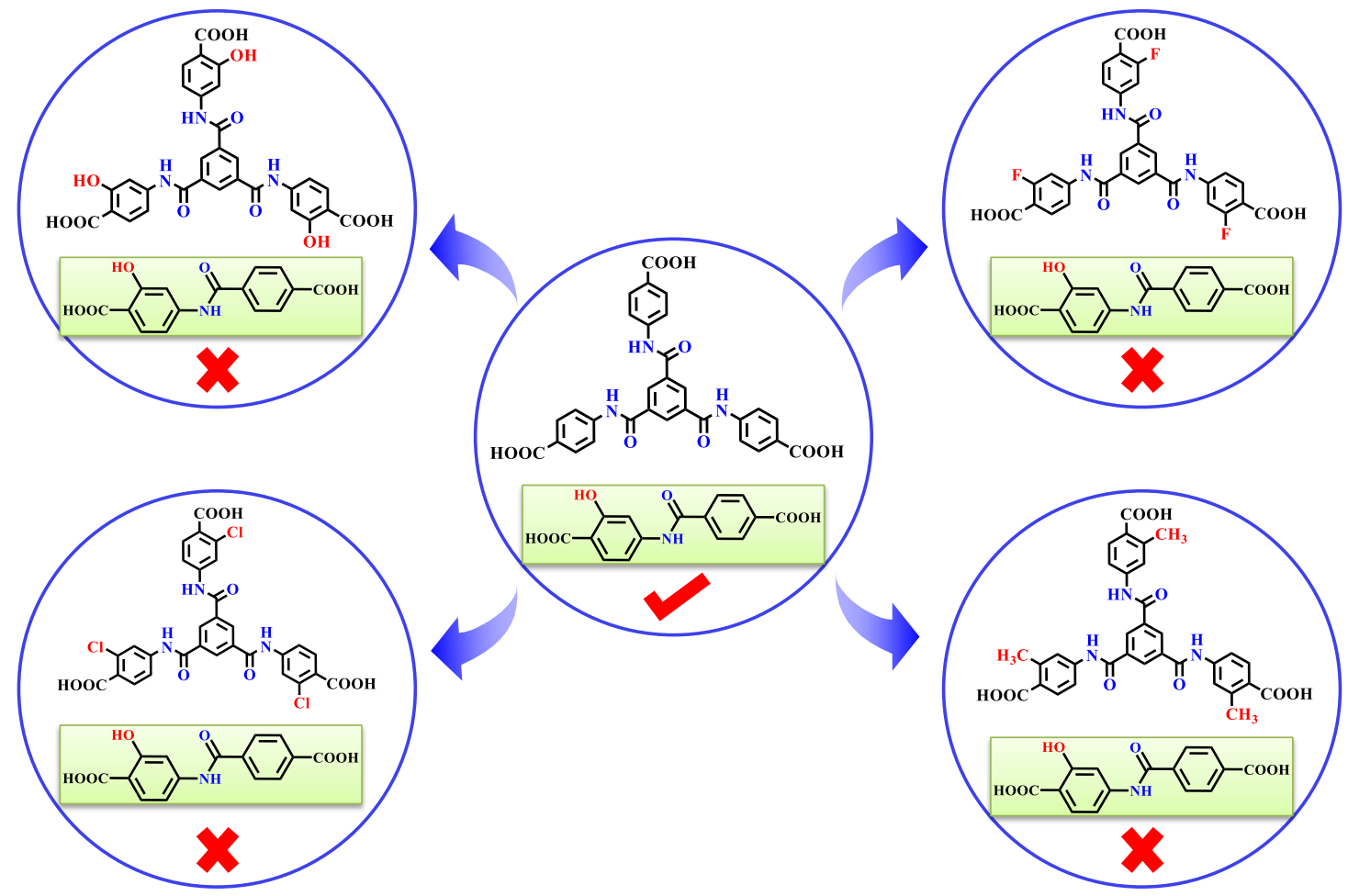

Figure S16. Schematic representation showing the appreciable $\mathrm{H}_{2} \mathrm{~L}_{2}-\mathrm{OH}$ combined with tritopic $\mathrm{H}_{3} \mathrm{~L}_{1}$ rather than $\mathrm{H}_{3} \mathrm{~L}_{1}-(\mathrm{OH})_{3}, \mathrm{H}_{3} \mathrm{~L}_{1}-\mathrm{F}_{3}, \mathrm{H}_{3} \mathrm{~L}_{1}-(\mathrm{Cl})_{3}$ and $\mathrm{H}_{3} \mathrm{~L}_{1}-\left(\mathrm{CH}_{3}\right)_{3}$ to isolate the intricate $3 \mathrm{~W}-\mathrm{ROD}-2-\mathrm{OH}$. 

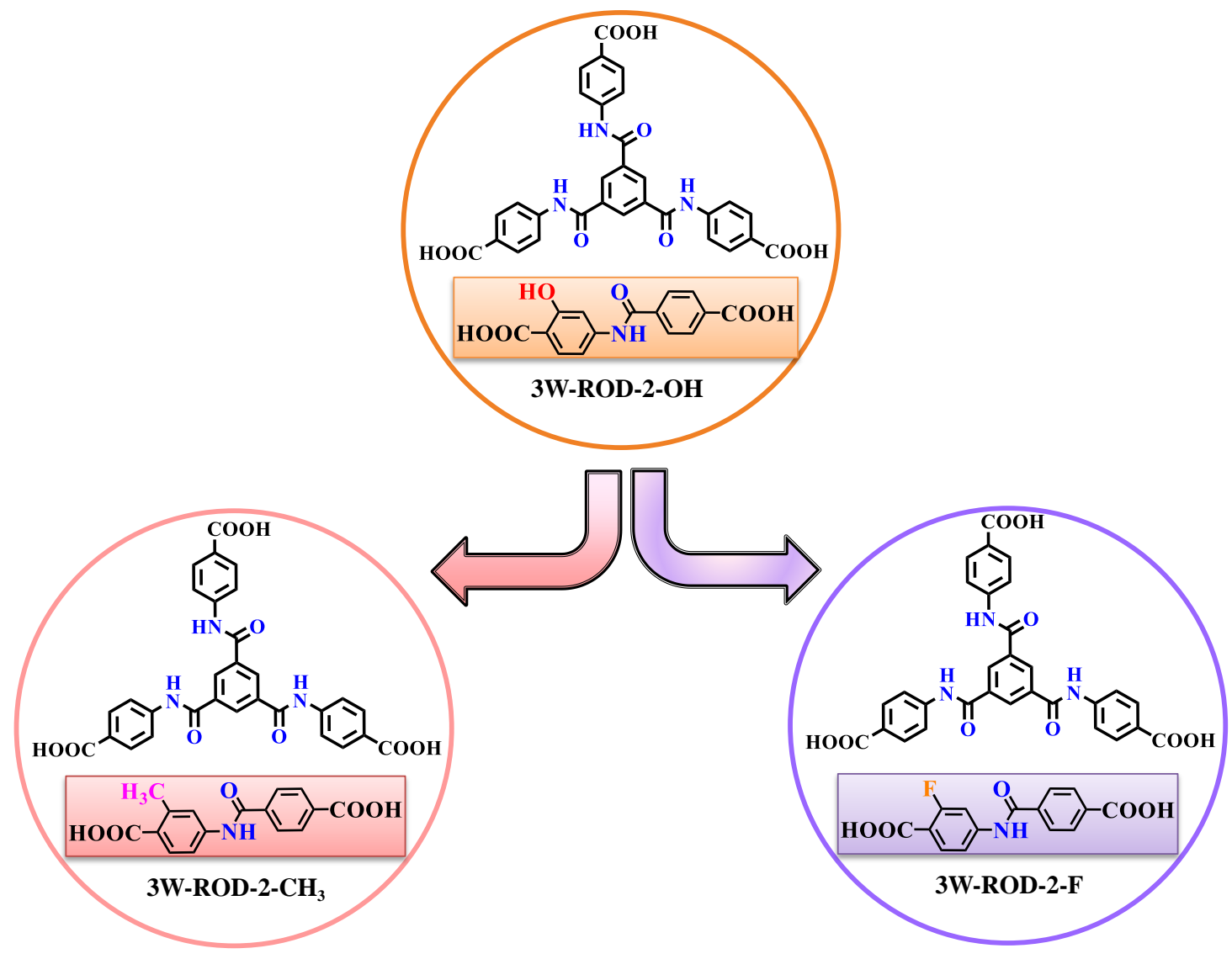

Figure S17. Schematic representation showing all the successful combinations of the $\mathrm{H}_{3} \mathrm{~L}_{1}$ with different ditopic linkers of $\mathrm{H}_{2} \mathrm{~L}_{2}-\mathrm{OH}$ and $\mathrm{H}_{2} \mathrm{~L}_{2}-\mathrm{F}$ as well as $\mathrm{H}_{2} \mathrm{~L}_{2}-\mathrm{CH}_{3}$ to isolate isoreticular 3W-ROD-2-X platform.
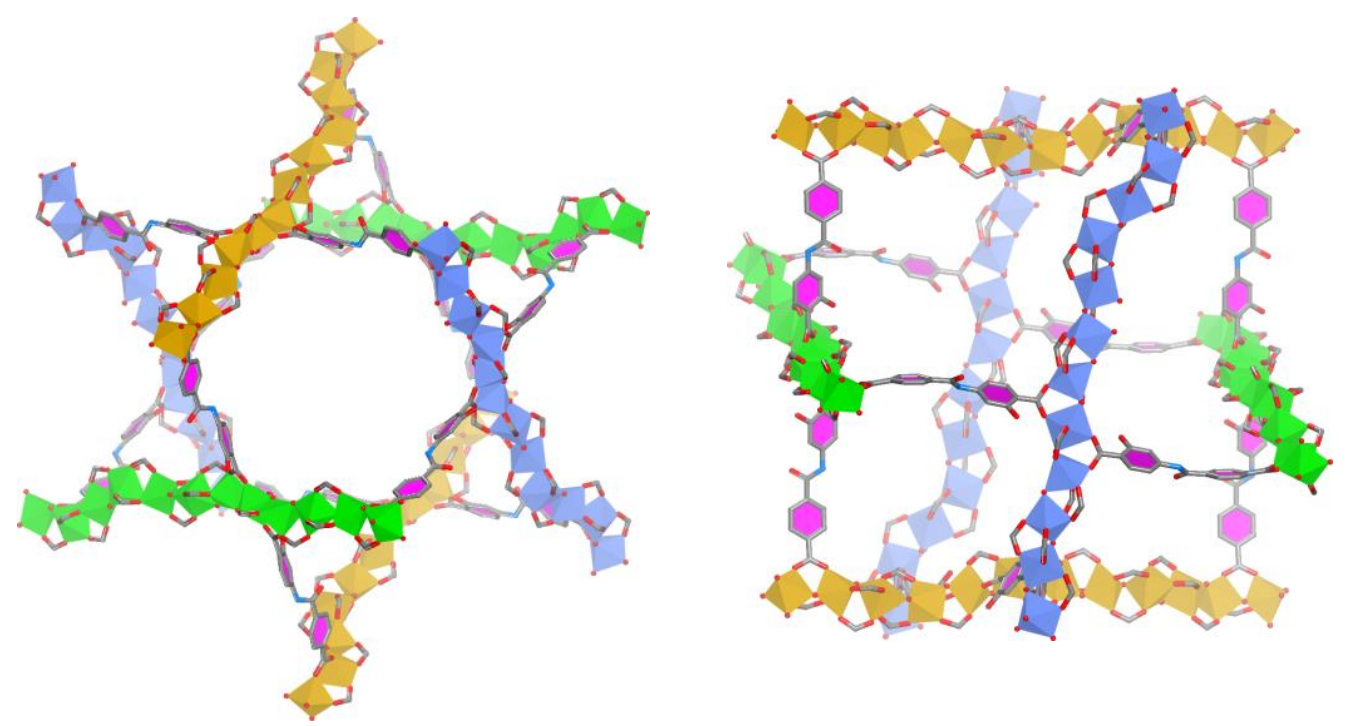

Figure S18. Schematic representation showing that merely $\mathrm{L}_{2}-\mathrm{OH}^{2-}$ linkers to sustain the three-way trigonal rod pattern is obviously impossible once deleting the $\mathrm{D}_{3}{ }^{6-}$ and $\mathrm{D}_{4}{ }^{6-}$ linkers within $3 \mathrm{~W}-\mathrm{ROD}-2-\mathrm{OH}$. 
(a)

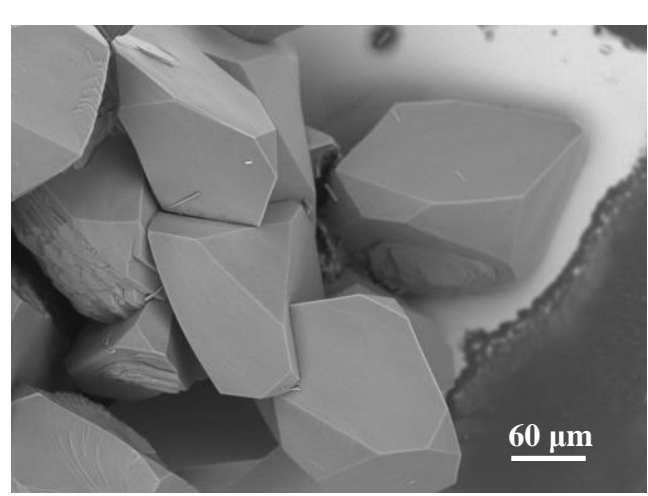

(c)

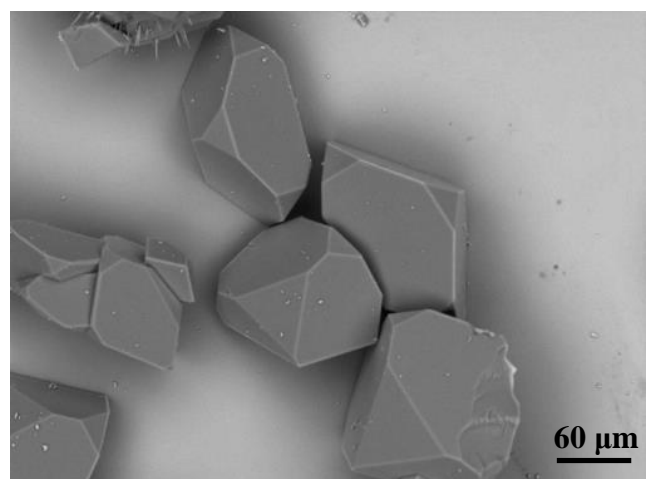

(b)

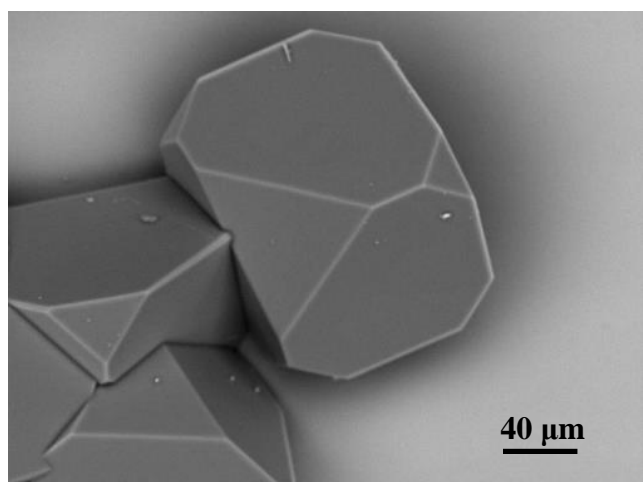

(d)

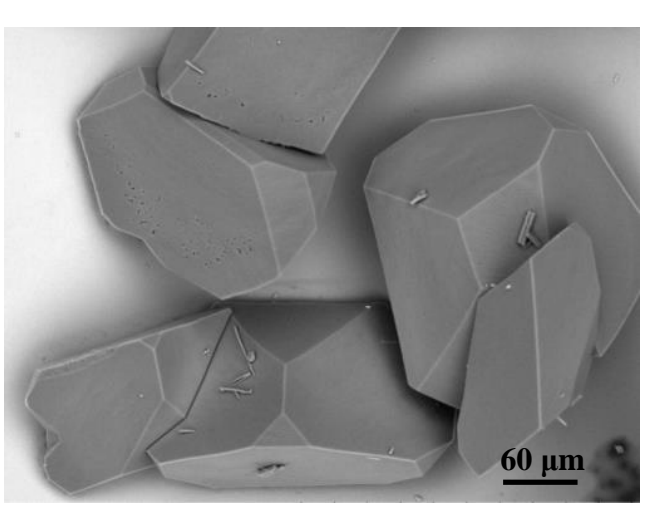

Figure S19. SEM images of 3W-ROD-1 (a), 3W-ROD-2-OH (b), 3W-ROD-2-F (c) and $3 \mathrm{~W}-\mathrm{ROD}-2-\mathrm{CH}_{3}(\mathrm{~d})$. 


\section{Section 5. PXRD and TGA Plots}

(a)

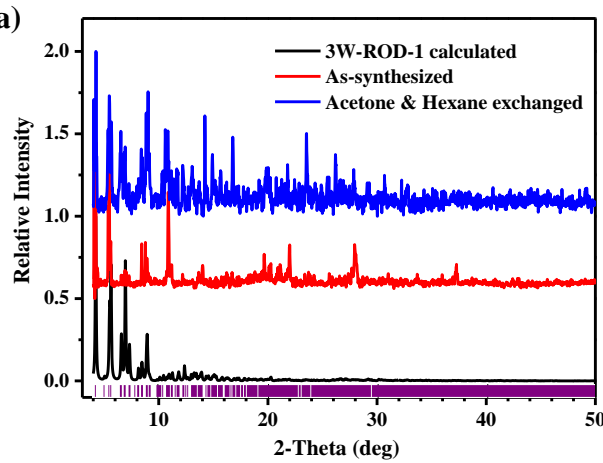

(c)

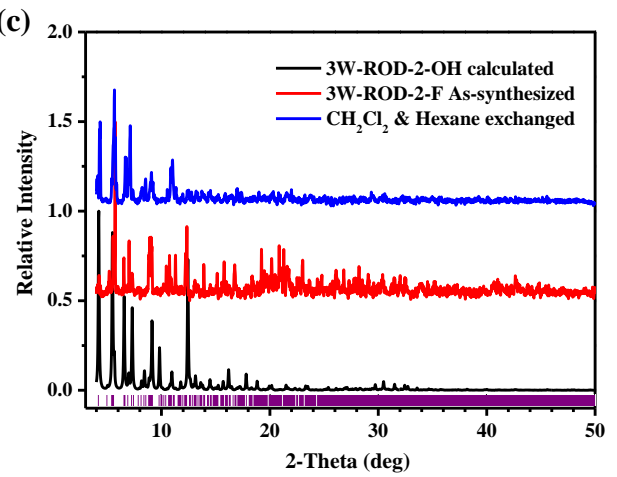

(b)

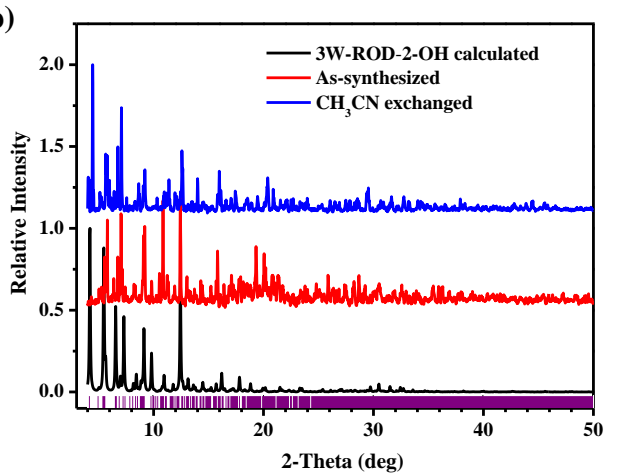

(d)

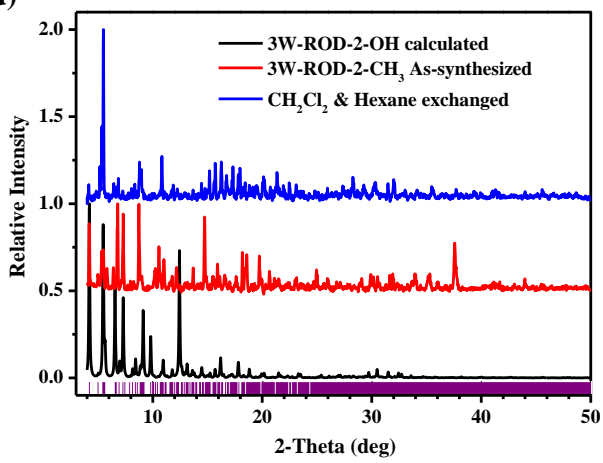

Figure S20. PXRD patterns of the calculated and as-synthesized as well as solvent exchanged compounds of $3 \mathrm{~W}-\mathrm{ROD}-1$ and $3 \mathrm{~W}-\mathrm{ROD}-2-\mathrm{X}$, indicating the phase purities of as-synthesized and solvent-exchanged samples (3W-ROD-2-F and 3WROD-2- $\mathrm{CH}_{3}$ were precisely validated by the excellent matching PXRD patterns between the as-synthesized bulk materials and the calculated 3W-ROD2-OH). 


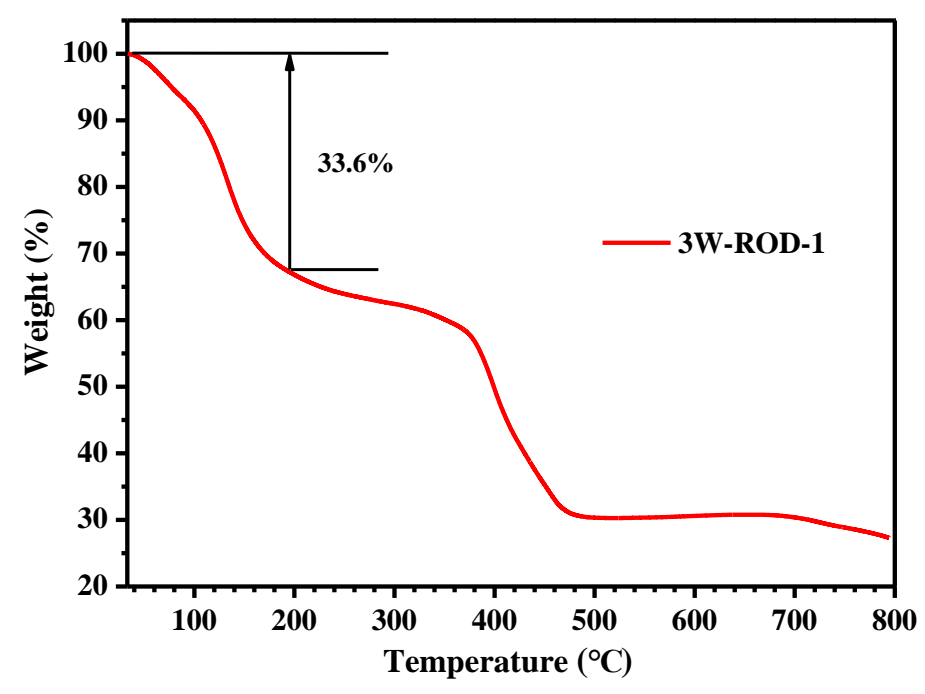

Figure S21. TGA plot of the as-synthesized $3 \mathrm{~W}-\mathrm{ROD}-1,<195{ }^{\circ} \mathrm{C}$, loss of free/surface/coordinated solvent/water; $>375{ }^{\circ} \mathrm{C}$, framework degradation.

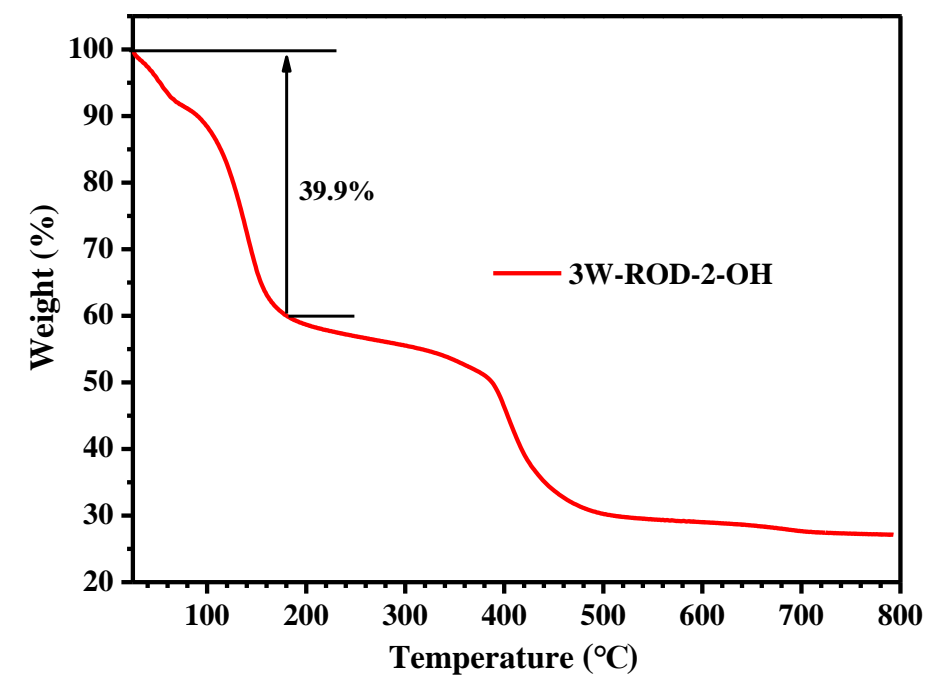

Figure S22. TGA plot of the as-synthesized 3W-ROD-2-OH, $<179{ }^{\circ} \mathrm{C}$, loss of free/surface water/DMF; $>375^{\circ} \mathrm{C}$, framework degradation. 


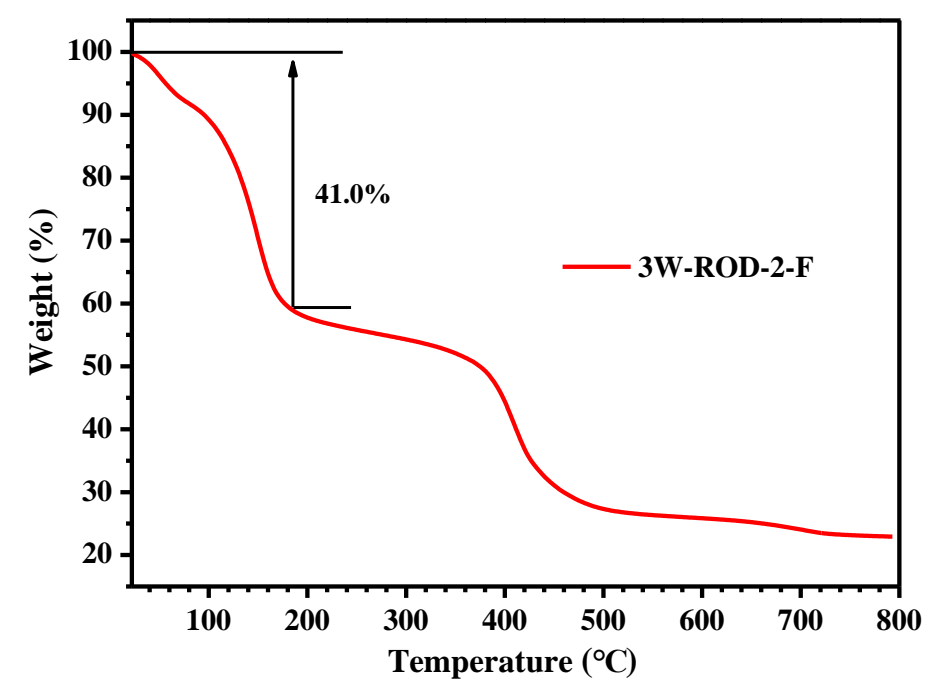

Figure S23. TGA plot of the as-synthesized $3 \mathrm{~W}-\mathrm{ROD}-2-\mathrm{F},<184{ }^{\circ} \mathrm{C}$, loss of free/surface water /DMF; $>371{ }^{\circ} \mathrm{C}$, framework degradation.

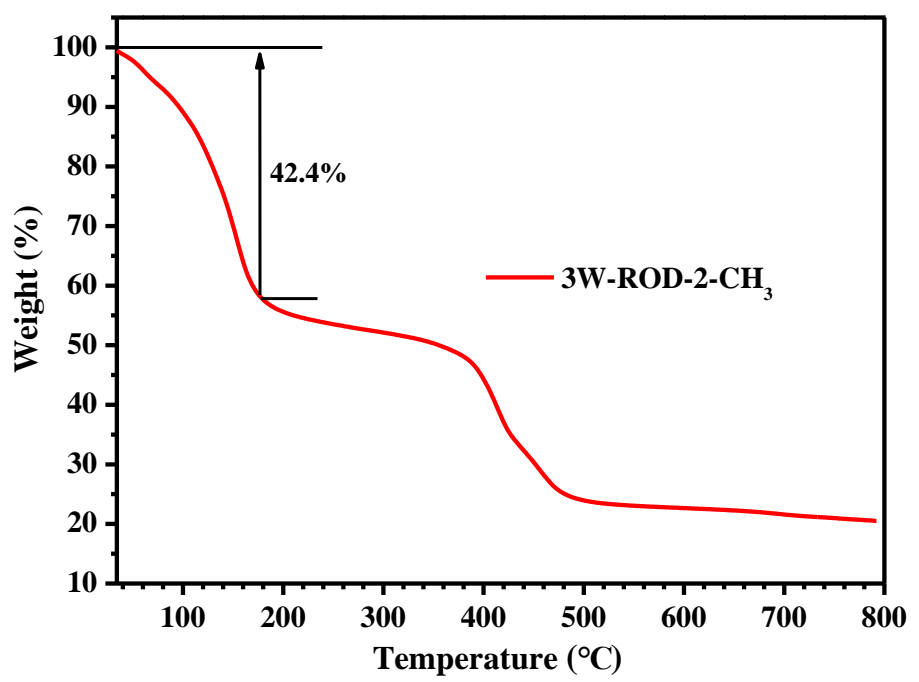

Figure S24. TGA plot of the as-synthesized $3 \mathrm{~W}-\mathrm{ROD}-2-\mathrm{CH}_{3},<177{ }^{\circ} \mathrm{C}$, loss of free/surface water/ DMF; $>380{ }^{\circ} \mathrm{C}$, framework degradation. 


\section{Section 6. Low Pressure Gas Sorption Isotherms and $Q_{\text {st }}$}

Sample activation. The as-synthesized sample of $3 \mathrm{~W}-\mathrm{ROD}-1$ was exchanged in acetone and $n$-hexane mixture $(4: 1)$ for 3 times during 6 hours, then removed in supercritical $\mathrm{CO}_{2}$ to give activated $3 \mathrm{~W}-\mathrm{ROD}-1$, in the supercritical $\mathrm{CO}_{2}$ process, the sample was exchanged with fresh liquid $\mathrm{CO}_{2}$ for three times, with a duration of 30 min each time. The activated sample was degassed under vacuum for $12 \mathrm{~h}$ at $303 \mathrm{~K}$ and refilled with $\mathrm{N}_{2}$ for storage. The as-synthesized sample of $3 \mathrm{~W}-\mathrm{ROD}-2-\mathrm{OH}$ were soaked in acetonitrile for 3 days with acetonitrile refreshing every 8 hours, then activated under vacuum at $303 \mathrm{~K}$ for 4 hours and then heated at $393 \mathrm{~K}$ for 10 hours. $3 \mathrm{~W}-\mathrm{ROD}-2-\mathrm{F}, 3 \mathrm{~W}-\mathrm{ROD}-2-\mathrm{CH}_{3}$ were soaked in dichloromethane for $120 \mathrm{~min}$ with dichloromethane refreshing every $40 \mathrm{~min}$, and afterwards, the samples were soaked in n-hexane for 120 min with $n$-hexane refreshing every $40 \mathrm{~min}^{2}{ }^{2}$ Then, the n-hexane exchanged samples of $3 \mathrm{~W}-\mathrm{ROD}-2-\mathrm{F}, 3 \mathrm{~W}-\mathrm{ROD}-2-\mathrm{CH}_{3}$ were activated under vacuum at $303 \mathrm{~K}$ for 4 hours and then heated at $373 \mathrm{~K}$ for 10 hours.

Low-pressure adsorption. Low pressure gas adsorption studies were conducted on a fully automated micropore gas analyzer Autosorb-iQ3 (Quantachrome Instruments) at relative pressures up to $1 \mathrm{~atm}$. The cryogenic temperature was controlled using liquid nitrogen at $77 \mathrm{~K}$. The bath temperature for the $\mathrm{CO}_{2}, \mathrm{CH}_{4}$ sorption measurements was controlled using a recirculating bath containing an ethylene glycol/ $\mathrm{H}_{2} \mathrm{O}$ mixture. The apparent surface areas were determined from the nitrogen adsorption isotherms collected at $77 \mathrm{~K}$ by applying the BET models. Pore size analyses were performed using a cylindrical/spherical NLDFT pore model system by assuming an oxidic (zeolitic) surface.

The isosteric enthalpy of adsorption, $Q_{\mathrm{st}}$ for $\mathrm{CO}_{2}$ was determined by fitting the adsorption isotherms at $273 \mathrm{~K}, 283 \mathrm{~K}$ and $298 \mathrm{~K}$ to the virial equation

$$
\ln P=\ln N+1 / T \sum_{i=0}^{m} a_{i} N^{i}+\sum_{j=0}^{n} b_{j} N^{j}
$$

Here, $P$ is the pressure in $\mathrm{Pa}, N$ is the amount adsorbed in $\mathrm{mmol} / \mathrm{g}, T$ is the temperature in $\mathrm{K}, a_{\mathrm{i}}$ and $b_{\mathrm{j}}$ are virial coefficients, and $m, n$ represent the number of coefficients required to adequately describe the isotherms ( $m$ and $n$ were gradually increased until the contribution of extra added $a$ and $b$ coefficients was deemed to be statistically insignificant towards the overall fit). The isosteric heats of adsorption $\left(Q_{\mathrm{st}}\right)$ [eq.(2)] were calculated from the temperature-dependent isotherms using the Clausius-Clapeyron equation [eq.(3)] as follows, where $P$ is the pressure in $\mathrm{Pa}, T$ is the temperature in $\mathrm{K}, R$ is the gas constant, and $n$ is the total amount adsorbed.

$$
\begin{aligned}
& -Q_{s t}=R T^{2}\left(\frac{\partial \ln P}{\partial T}\right)_{n} \\
& \frac{d \ln P}{d T}=\frac{\Delta H}{n R T^{2}}=\frac{\Delta_{r} H_{m}}{R T^{2}} .
\end{aligned}
$$




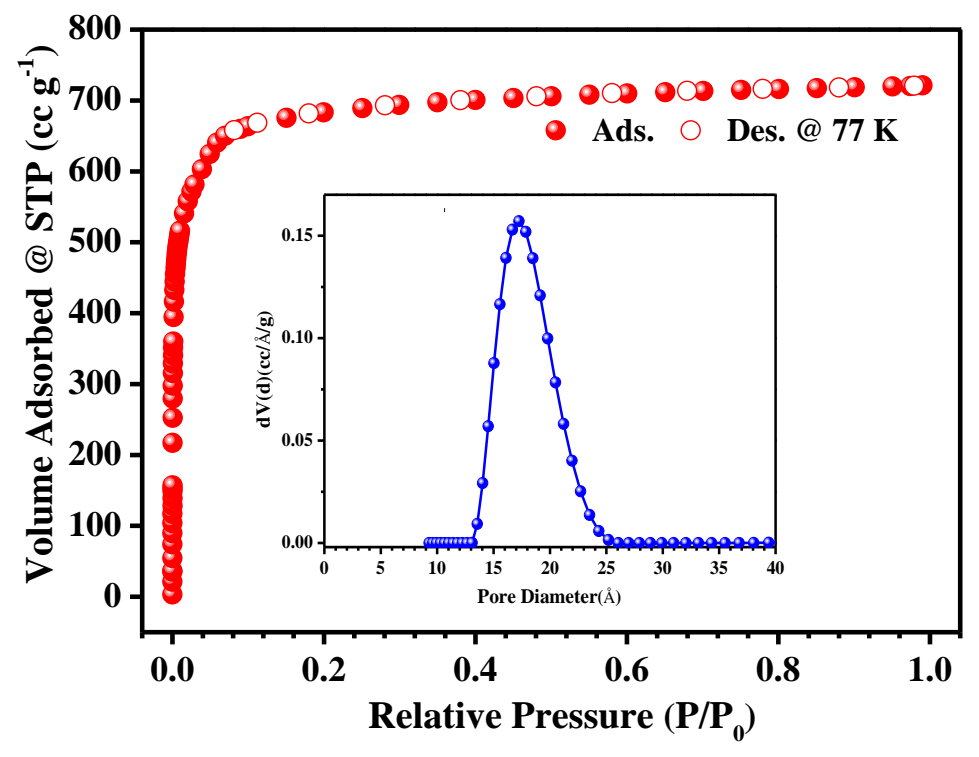

Figure S25. Low-pressure $\mathrm{N}_{2}$ adsorption isotherm at $77 \mathrm{~K}$ of $3 \mathrm{~W}-\mathrm{ROD}-1$ (filled symbols, adsorption; empty symbols, desorption); inset: pore size distribution analyzed by NLDFT method.

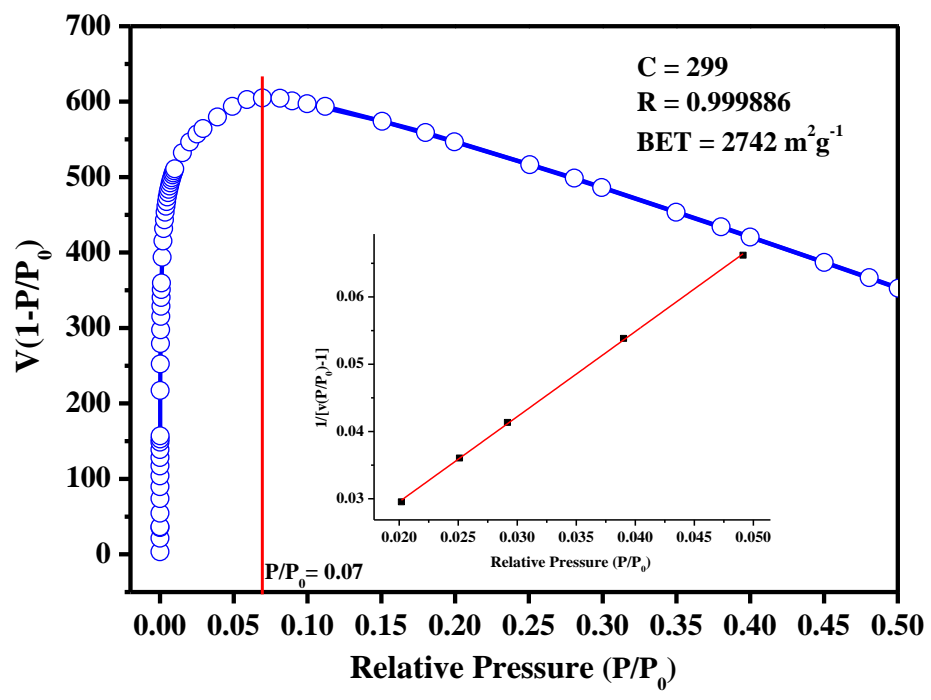

Figure S26. $V\left(1-P / P_{0}\right)$ vs. $P / P_{0}$ for $3 \mathrm{~W}-\mathrm{ROD}-1$, only the range below $P / P_{0}=0.07$ satisfies the first consistency criterion for applying the BET theory; inset: plot of the linear region for the BET equation. 


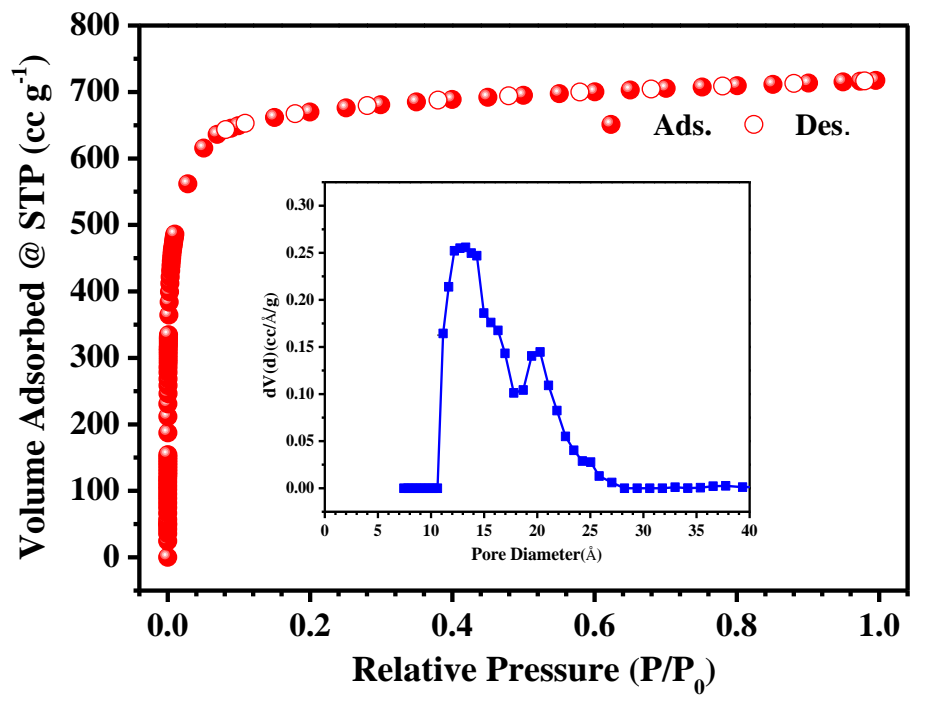

Figure S27. Low-pressure $\mathrm{N}_{2}$ adsorption isotherm at $77 \mathrm{~K}$ of $3 \mathrm{~W}-\mathrm{ROD}-2-\mathrm{OH}$ (filled symbols, adsorption; empty symbols, desorption); inset: pore size distribution analyzed by NLDFT method.

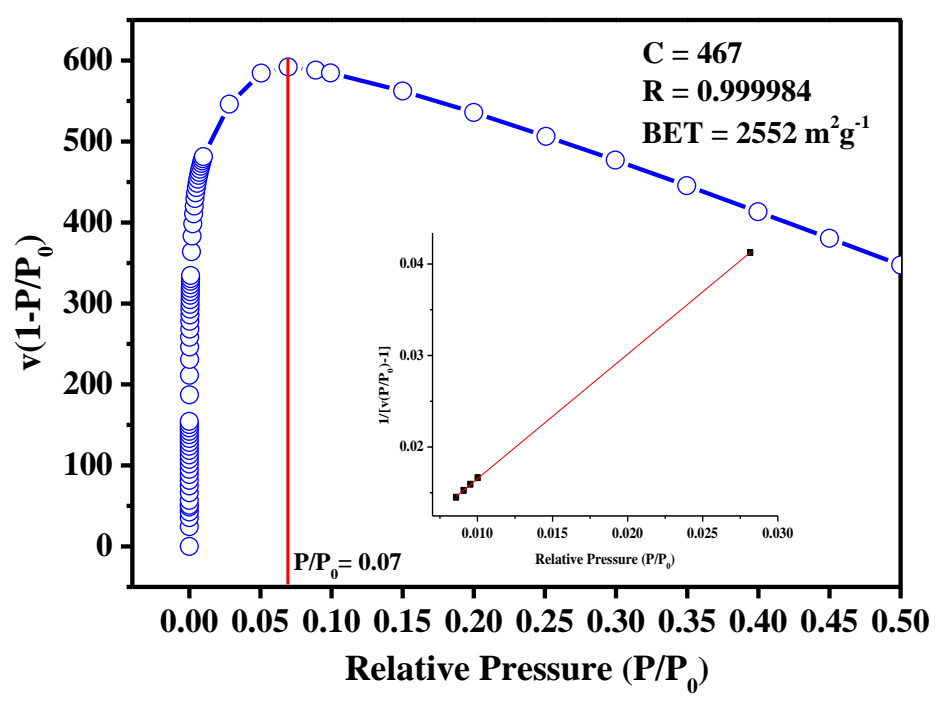

Figure S28. $V\left(1-P / P_{0}\right)$ vs. $P / P_{0}$ for $3 \mathrm{~W}-\mathrm{ROD}-2-\mathrm{OH}$, only the range below $P / P_{0}=0.07$ satisfies the first consistency criterion for applying the BET theory; inset: plot of the linear region for the BET equation. 


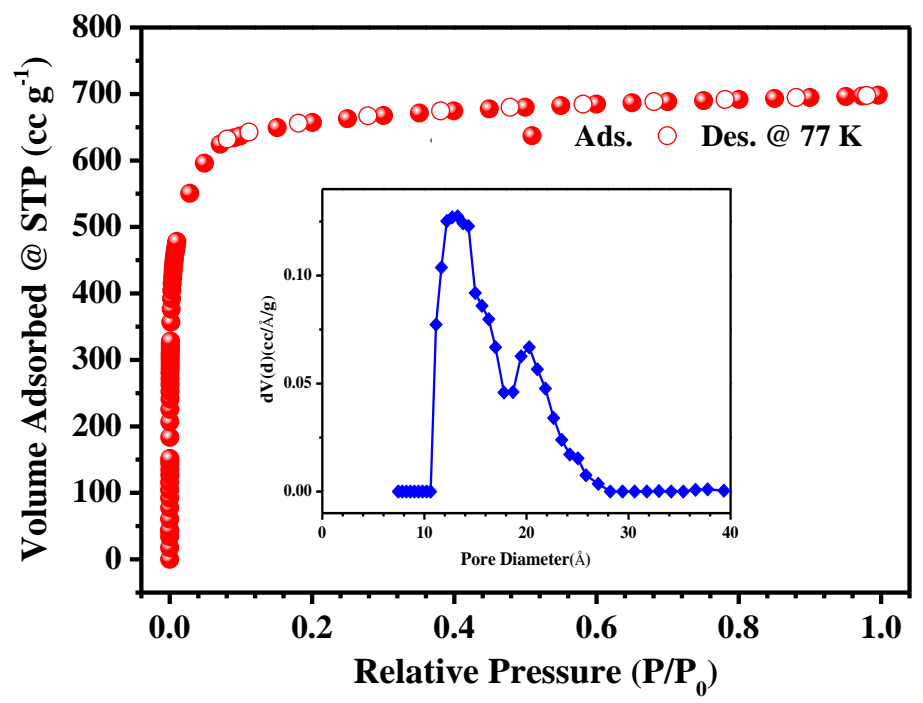

Figure S29. Low-pressure $\mathrm{N}_{2}$ adsorption isotherm at $77 \mathrm{~K}$ of $3 \mathrm{~W}-\mathrm{ROD}-2-\mathrm{F}$ (filled symbols, adsorption; empty symbols, desorption); inset: pore size distribution analyzed by NLDFT method.

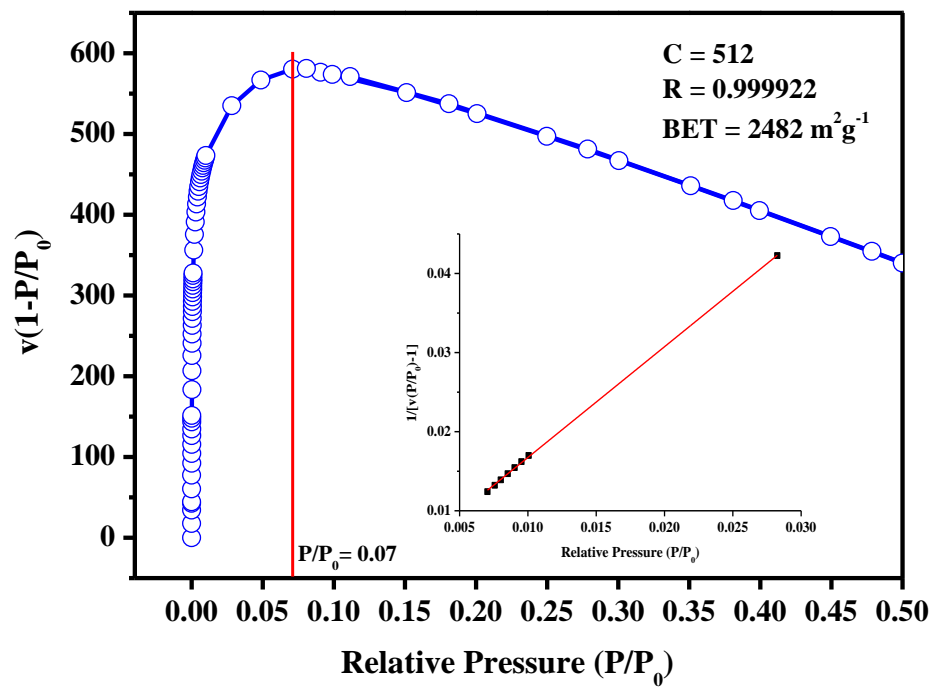

Figure S30. $V\left(1-P / P_{0}\right)$ vs. $P / P_{0}$ for $3 \mathrm{~W}-\mathrm{ROD}-2-\mathrm{F}$, only the range below $P / P_{0}=0.07$ satisfies the first consistency criterion for applying the BET theory; inset: plot of the linear region for the BET equation. 


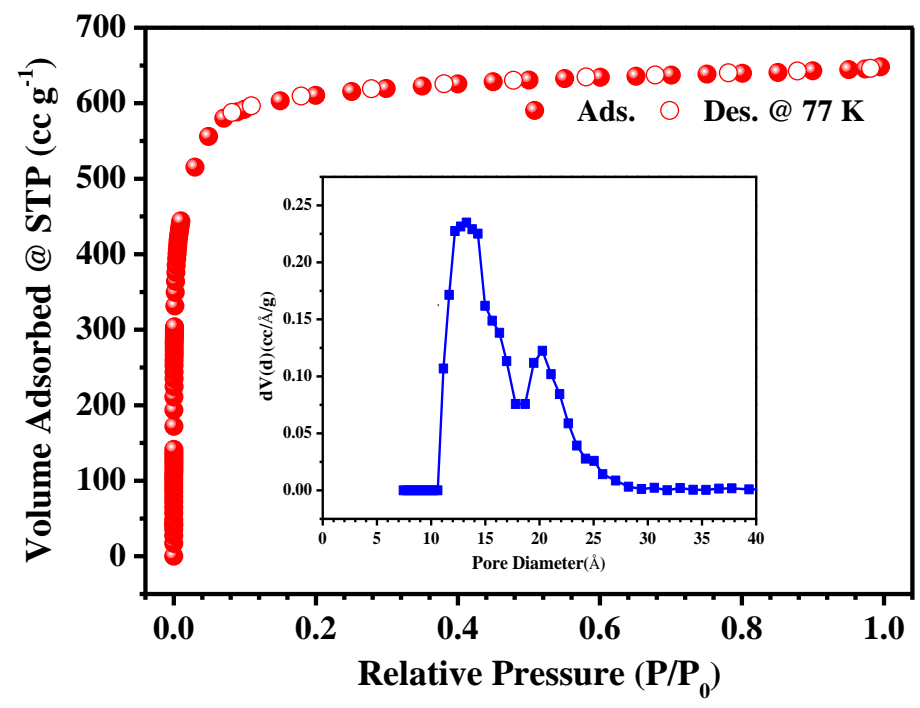

Figure S31. Low-pressure $\mathrm{N}_{2}$ adsorption isotherm at $77 \mathrm{~K}$ of $3 \mathrm{~W}-\mathrm{ROD}-2-\mathrm{CH}_{3}$ (filled symbols, adsorption; empty symbols, desorption); inset: pore size distribution analyzed by NLDFT method.

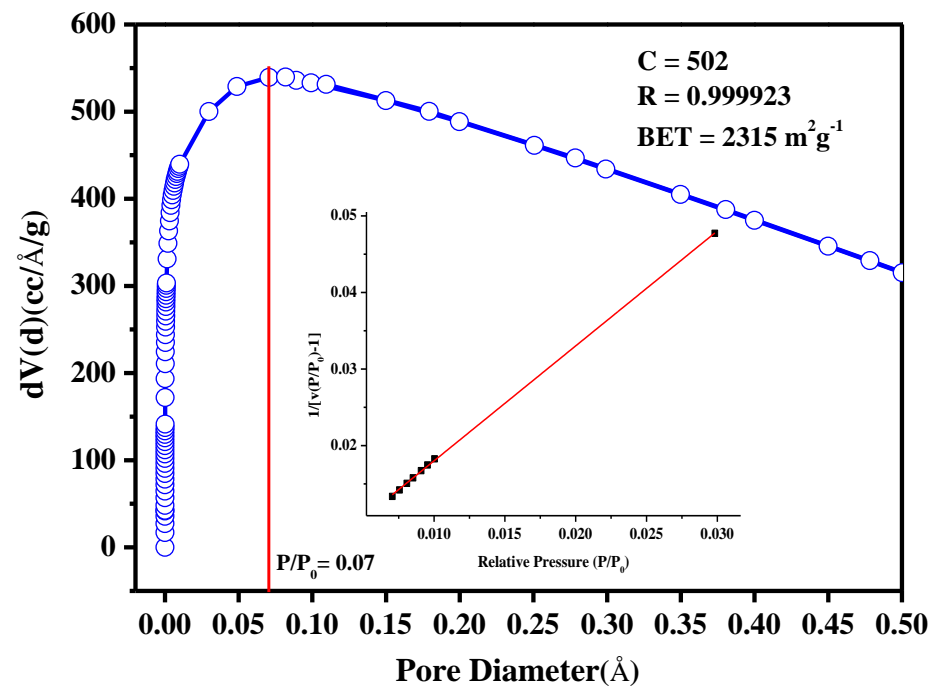

Figure S32. $V\left(1-P / P_{0}\right)$ vs. $P / P_{0}$ for $3 \mathrm{~W}-\mathrm{ROD}-2-\mathrm{CH}_{3}$, only the range below $P / P_{0}=$ 0.07 satisfies the first consistency criterion for applying the BET theory; inset: plot of the linear region for the BET equation. 
Table S1. Some typical rod MOFs and corresponding BET values.

\begin{tabular}{|c|c|c|c|c|}
\hline $\begin{array}{c}\text { Rod packing } \\
\text { pattern }\end{array}$ & Name & $\begin{array}{c}\text { BET } \\
\left(\mathrm{m}^{2} \mathrm{~g}^{-1}\right)\end{array}$ & $\begin{array}{l}\text { Pore Volume } \\
\left(\mathrm{cm}^{3} \mathrm{~g}^{-1}\right)\end{array}$ & Reference \\
\hline \multirow{27}{*}{ One-Way } & CYCU-3 & 2757 & 1.39 & 3 \\
\hline & $\mathrm{Co}(\mathrm{BDP})$ & $2670^{\mathrm{a}}$ & 0.93 & 4 \\
\hline & Ni-MOF-74 & 1350 & 0.51 & 5 \\
\hline & IR-MOF-74- II & 2510 & 1.04 & 6 \\
\hline & IR-MOF-74-III & 2440 & 1.23 & 6 \\
\hline & IR-MOF-74-IV & 2480 & 1.60 & 6 \\
\hline & IR-MOF-74- V & 2230 & 1.89 & 6 \\
\hline & IR-MOF-74-VI & 1600 & 1.65 & 6 \\
\hline & IR-MOF-74-VII & 1800 & 2.12 & 6 \\
\hline & IR-MOF-74-VIII & 1920 & 2.51 & 6 \\
\hline & IR-MOF-74-IX & 1760 & 3.41 & 6 \\
\hline & MOF-910 & 2120 & NA & 7 \\
\hline & 437-MOF & 2379 & 1.11 & 8 \\
\hline & MIL-53(Al)-OH & 1284 & NA & 9 \\
\hline & MIL-53(Al)-(OH) & 1193 & $\mathrm{NA}$ & 9 \\
\hline & MIL-120 & 308 & 0.11 & 10 \\
\hline & MIL-103 & 930 & NA & 11 \\
\hline & VNU-21 & 1440 & 0.58 & 12 \\
\hline & VNU-22 & 1030 & 0.41 & 12 \\
\hline & MIL-140-A & $415(10)$ & 0.18 & 13 \\
\hline & MIL-140-B & $460(10)$ & 0.18 & 13 \\
\hline & MIL-140-C & $670(20)$ & 0.27 & 13 \\
\hline & MIL-140-D & $701(20)$ & 0.29 & 13 \\
\hline & $\mathrm{In}_{2}(\mathrm{OH})_{2}(\mathrm{TBAPy})$ & 1189 & 0.639 & 14 \\
\hline & FIR-5 & 950.8 & 0.472 & 15 \\
\hline & $\mathrm{Fe}_{2}(\mathrm{BDP})_{3}$ & 1230 & NA & 16 \\
\hline & $\mathrm{La}(\mathrm{BTB})$ & 1024 & 0.416 & 17 \\
\hline \multirow{5}{*}{ Two-Way } & CAU-8 & 1078 & 0.46 & 18 \\
\hline & CAU-8-ODB & 1004 & 0.47 & 19 \\
\hline & $\mathrm{Zn}_{3}(\mathrm{BTP})_{2}$ & 930 & NA & 20 \\
\hline & MOF-77 & $\mathrm{NA}$ & NA & 21 \\
\hline & $\mathrm{Cd}_{3}(\mathrm{BPT})_{2}$ & NA & NA & 22 \\
\hline \multirow{5}{*}{ Three-Way } & $\mathrm{Zn}_{6}\left(\mathrm{H}_{2} \mathrm{O}\right)_{3}(\mathrm{BTP})_{4}$ & NA & NA & 20 \\
\hline & 3W-ROD-1 & 2742 & 1.11 & This work \\
\hline & 3W-ROD-2-OH & 2552 & 1.11 & This work \\
\hline & 3W-ROD-2-F & 2482 & 1.08 & This work \\
\hline & 3W-ROD-2-CH 3 & 2315 & 1.00 & This work \\
\hline
\end{tabular}

NA: not available; a: Langmuir surface area 


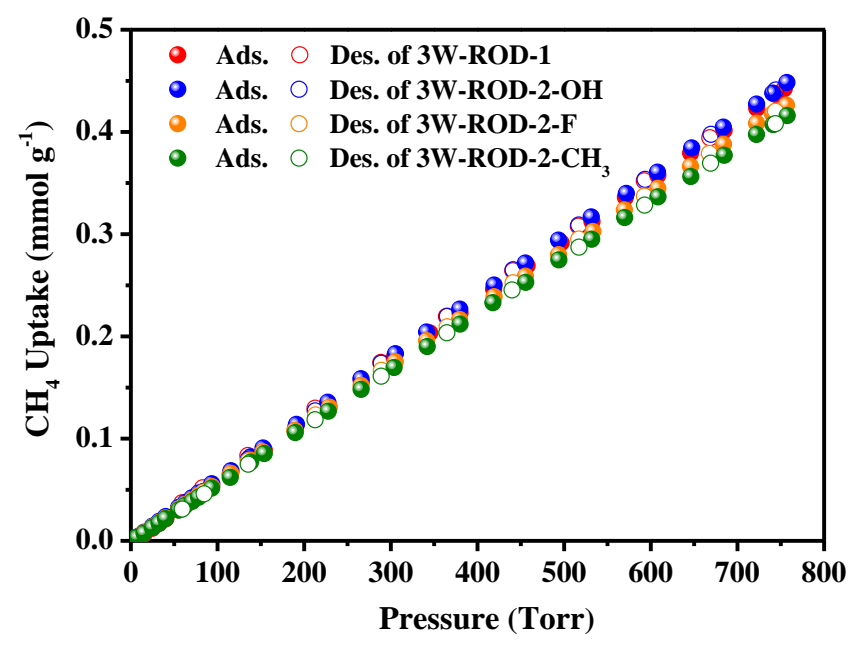

Figure S33. Low-pressure $\mathrm{CH}_{4}$ adsorption isotherms for $3 \mathrm{~W}-\mathrm{ROD}-1,3 \mathrm{~W}-\mathrm{ROD}-2-\mathrm{X}$ $\left(\mathrm{X}=-\mathrm{OH},-\mathrm{F}\right.$ and $\left.-\mathrm{CH}_{3}\right)$ measured at $298 \mathrm{~K}$.

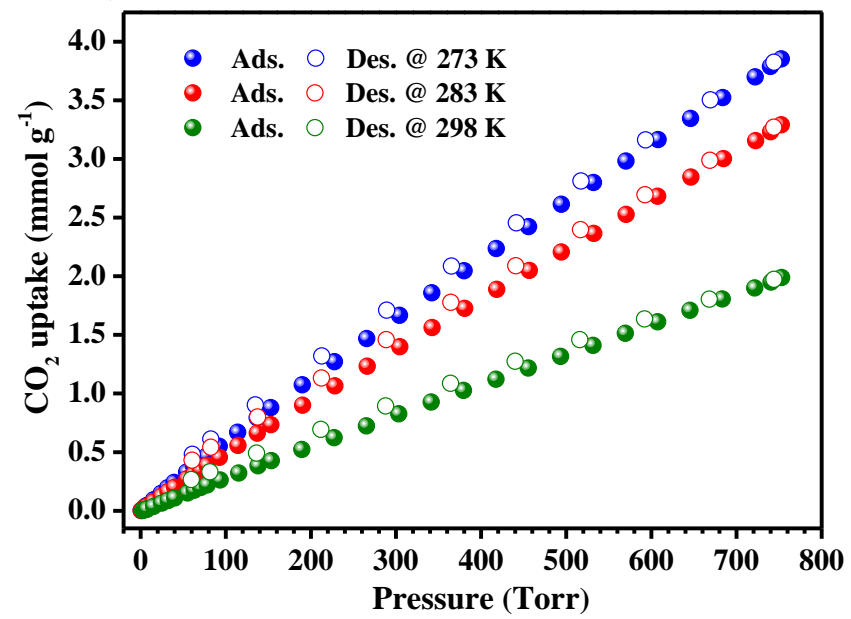

Figure S34. $\mathrm{CO}_{2}$ sorption isotherms for 3W-ROD-1 measured at 273, 283 and $298 \mathrm{~K}$, respectively.

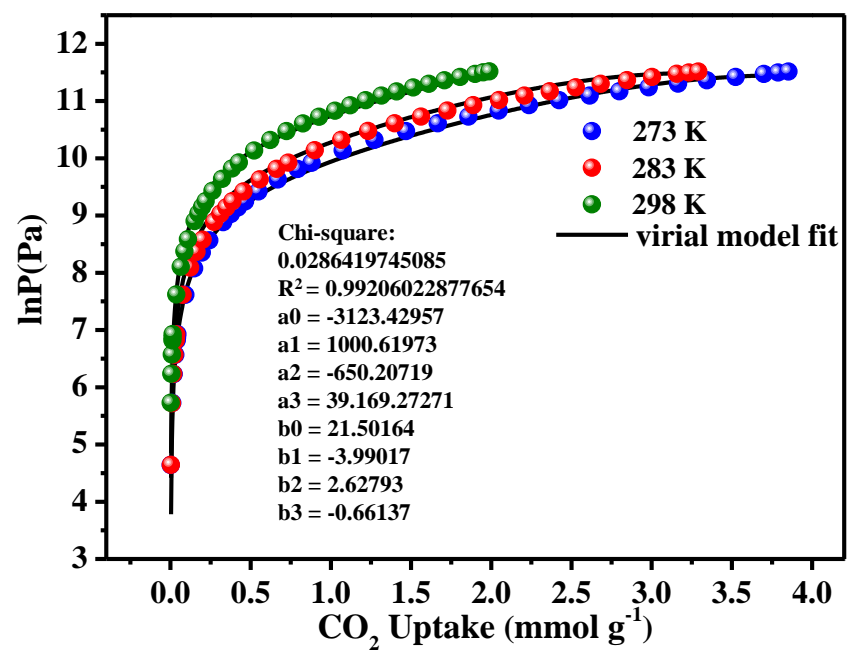

Figure S35. Virial fitting of the variable-temperature $\mathrm{CO}_{2}$ adsorption isotherms for 3W-ROD-1. 


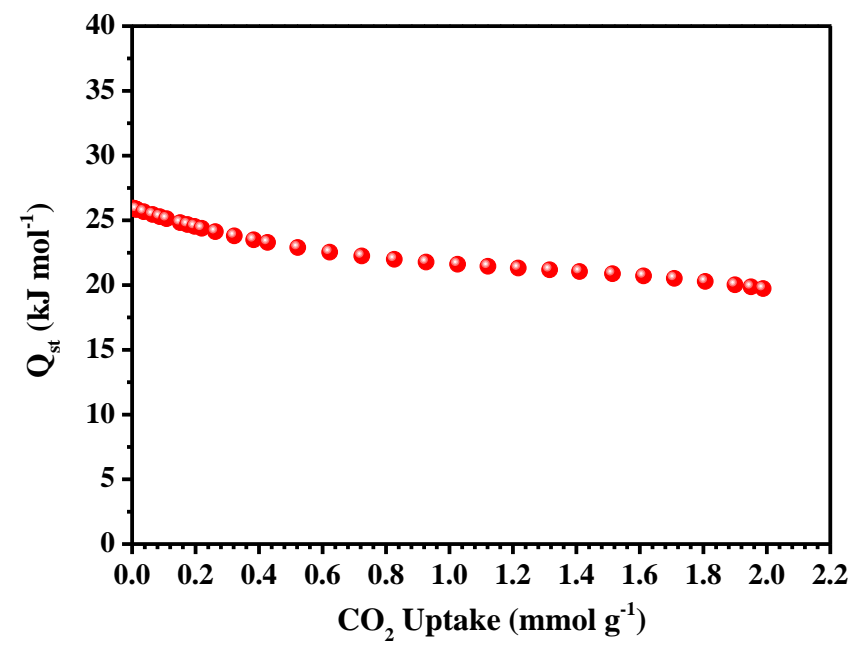

Figure S36. Calculated $\mathrm{CO}_{2} Q_{\text {st }}$ for $3 \mathrm{~W}-\mathrm{ROD}-1$.

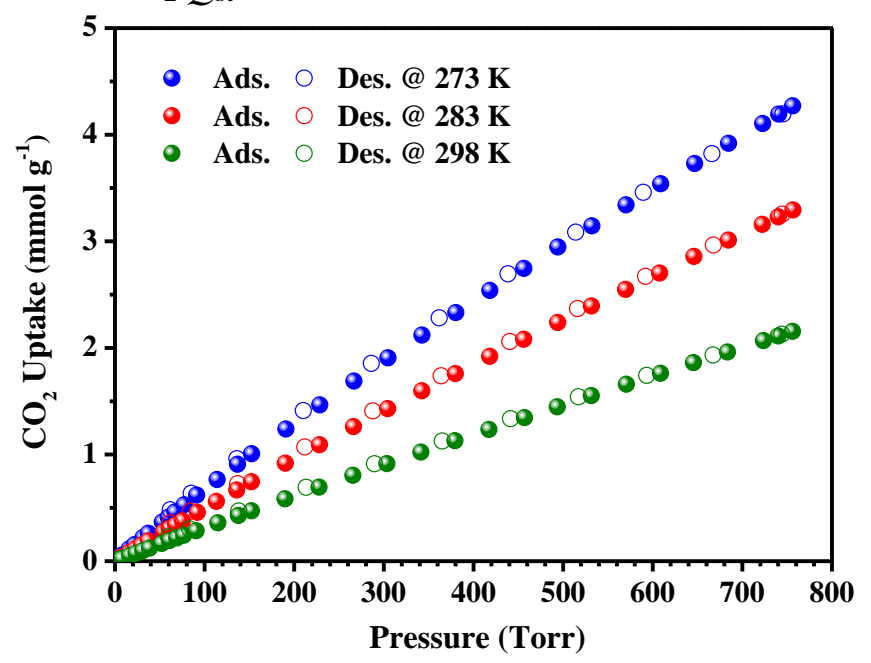

Figure S37. $\mathrm{CO}_{2}$ sorption isotherms for 3W-ROD-2-OH measured at 273, 283 and $298 \mathrm{~K}$, respectively.

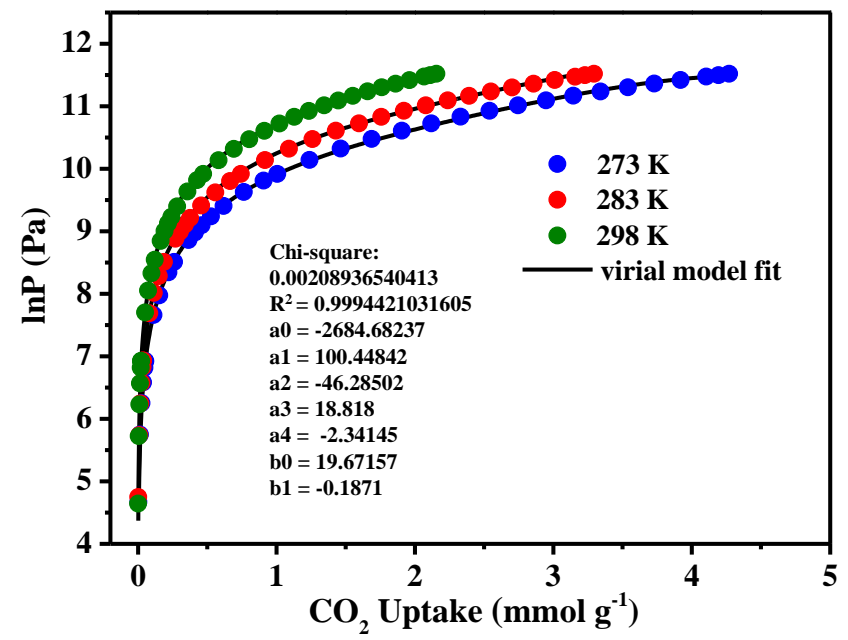

Figure S38. Virial fitting of the temperature-dependent $\mathrm{CO}_{2}$ adsorption isotherms for 3W-ROD-2-OH. 


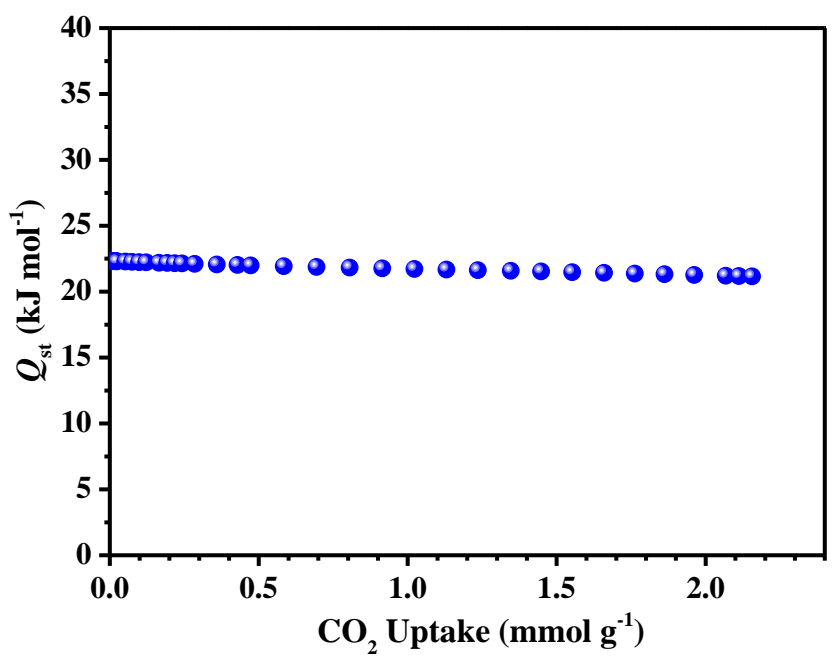

Figure S39. Calculated $\mathrm{CO}_{2} Q_{\mathrm{st}}$ for $3 \mathrm{~W}-\mathrm{ROD}-2-\mathrm{OH}$.

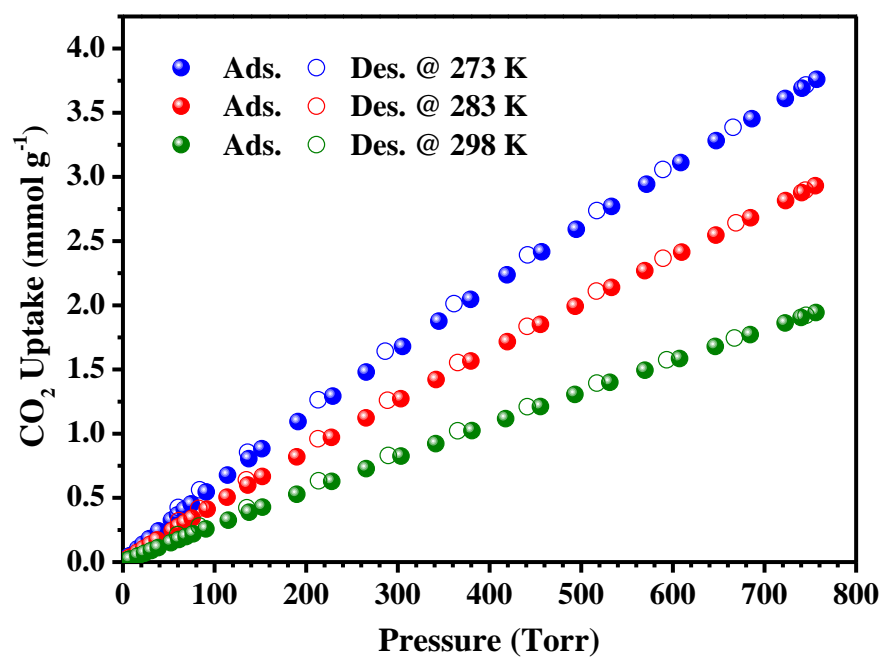

Figure S40. $\mathrm{CO}_{2}$ sorption isotherms for 3W-ROD-2-F measured at 273, 283 and 298 $\mathrm{K}$, respectively.

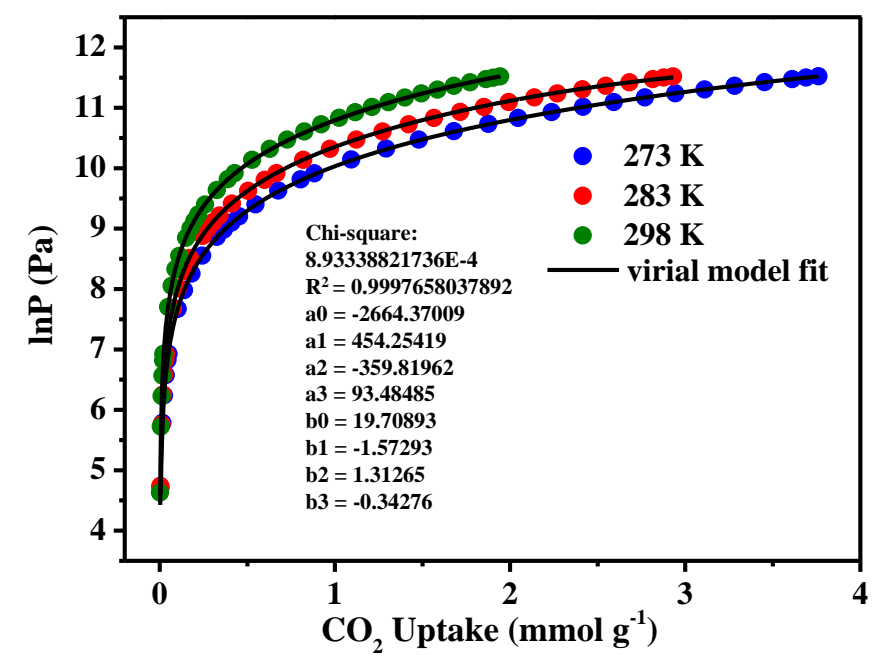

Figure S41. Virial fitting of the temperature-dependent $\mathrm{CO}_{2}$ adsorption isotherms for 3W-ROD-2-F. 


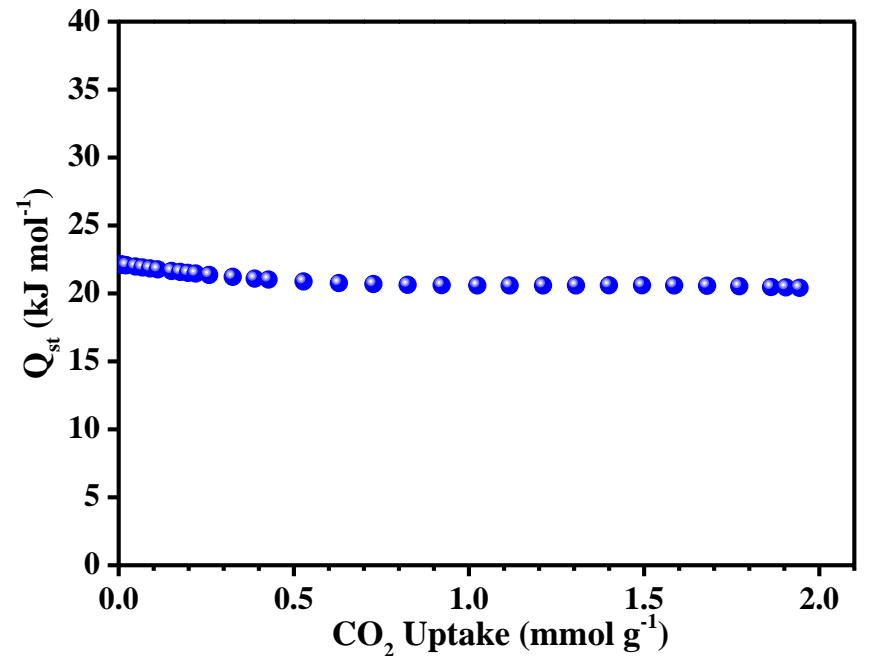

Figure S42. Calculated $\mathrm{CO}_{2} Q_{\text {st }}$ for $3 \mathrm{~W}-\mathrm{ROD}-2-\mathrm{F}$.

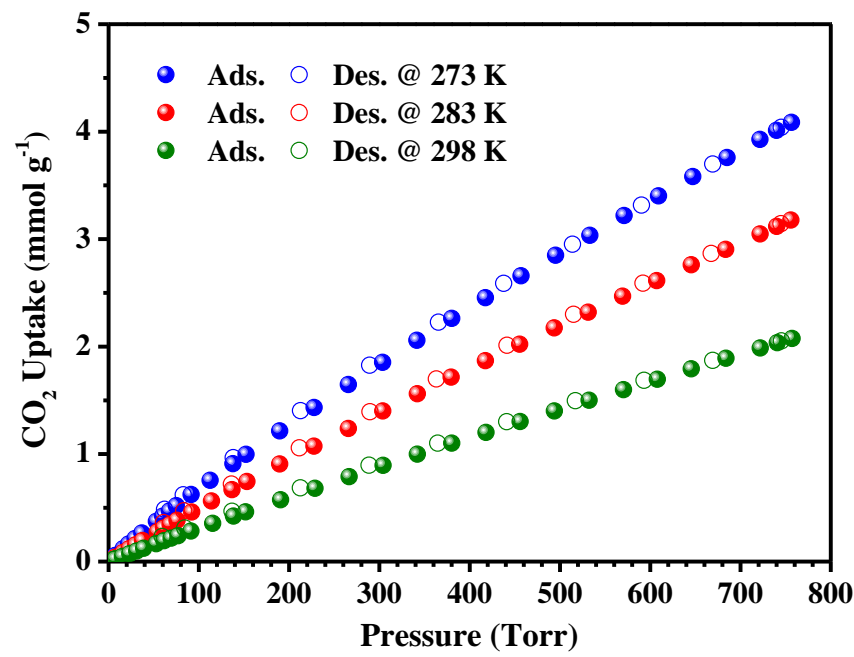

Figure S43. $\mathrm{CO}_{2}$ sorption isotherms for $3 \mathrm{~W}-\mathrm{ROD}-2-\mathrm{CH}_{3}$ measured at 273,283 and $298 \mathrm{~K}$, respectively.

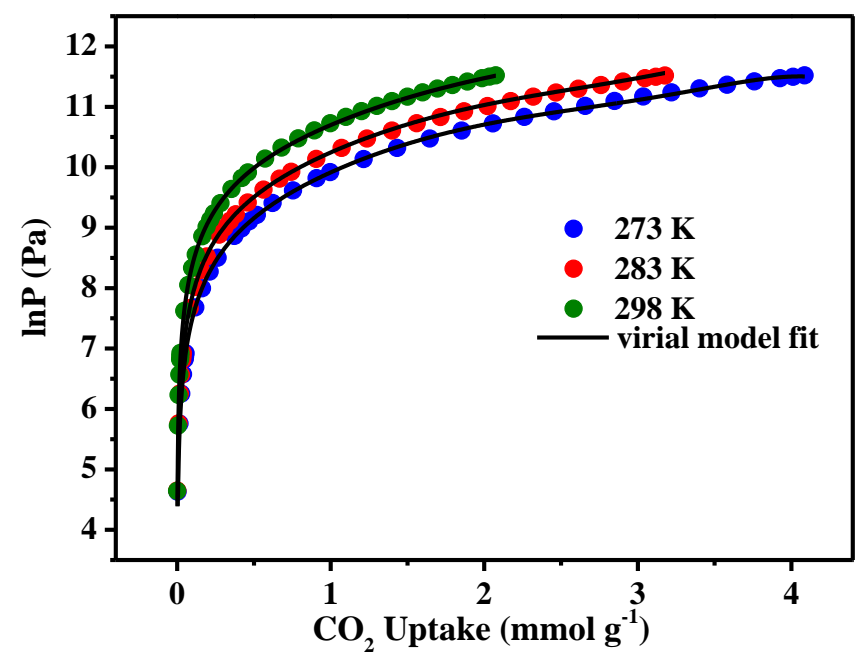

Figure S44. Virial fitting of the temperature-dependent $\mathrm{CO}_{2}$ adsorption isotherms for $3 \mathrm{~W}-\mathrm{ROD}-2-\mathrm{CH}_{3}$. 


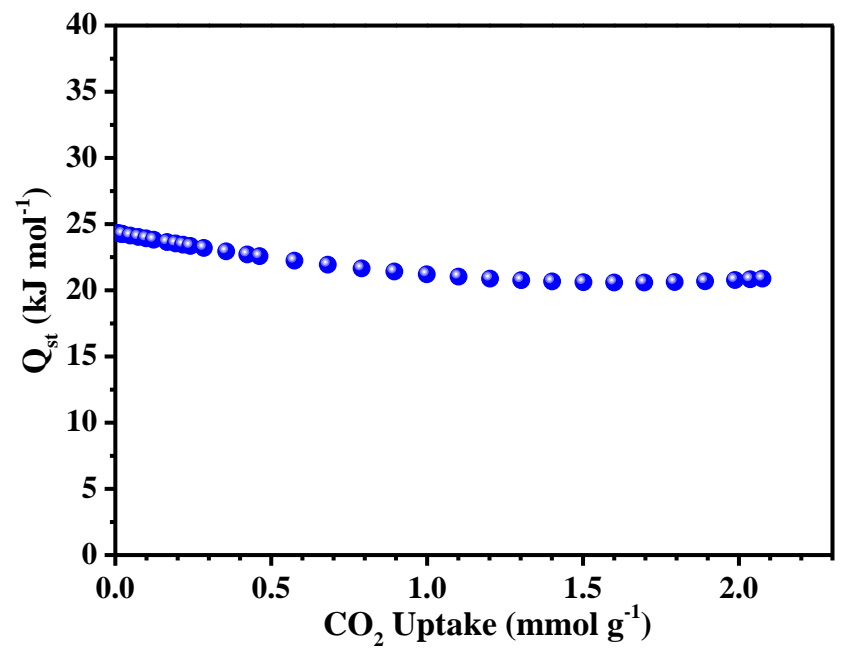

Figure S45. Calculated $\mathrm{CO}_{2} Q_{\text {st }}$ for $3 \mathrm{~W}-\mathrm{ROD}-2-\mathrm{CH}_{3}$.

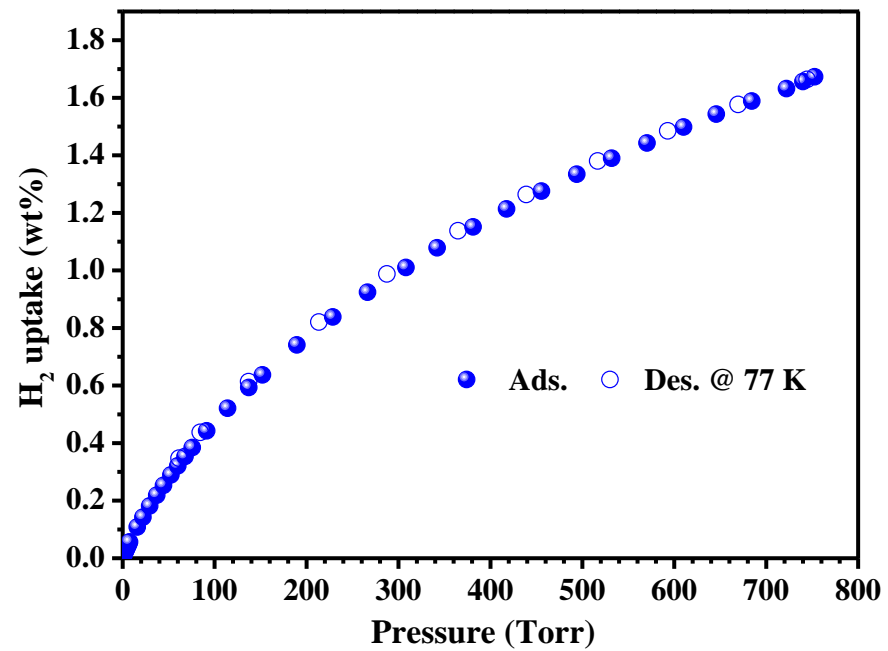

Figure S46. $\mathrm{H}_{2}$ sorption isotherm for $3 \mathrm{~W}-\mathrm{ROD}-1$ measured at $77 \mathrm{~K}$.

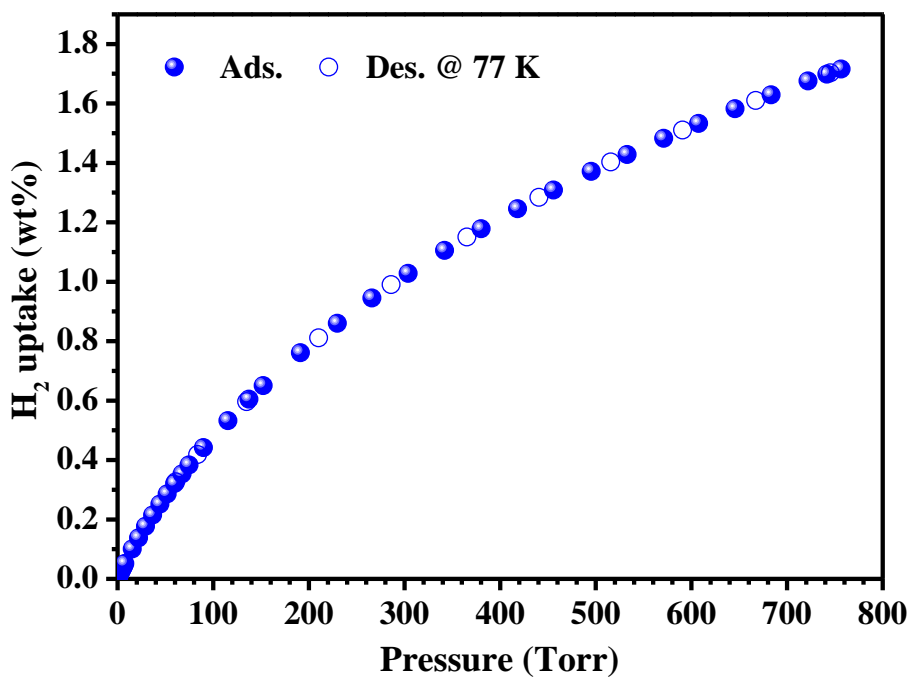

Figure S47. $\mathrm{H}_{2}$ sorption isotherm for $3 \mathrm{~W}-\mathrm{ROD}-2-\mathrm{OH}$ measured at $77 \mathrm{~K}$. 


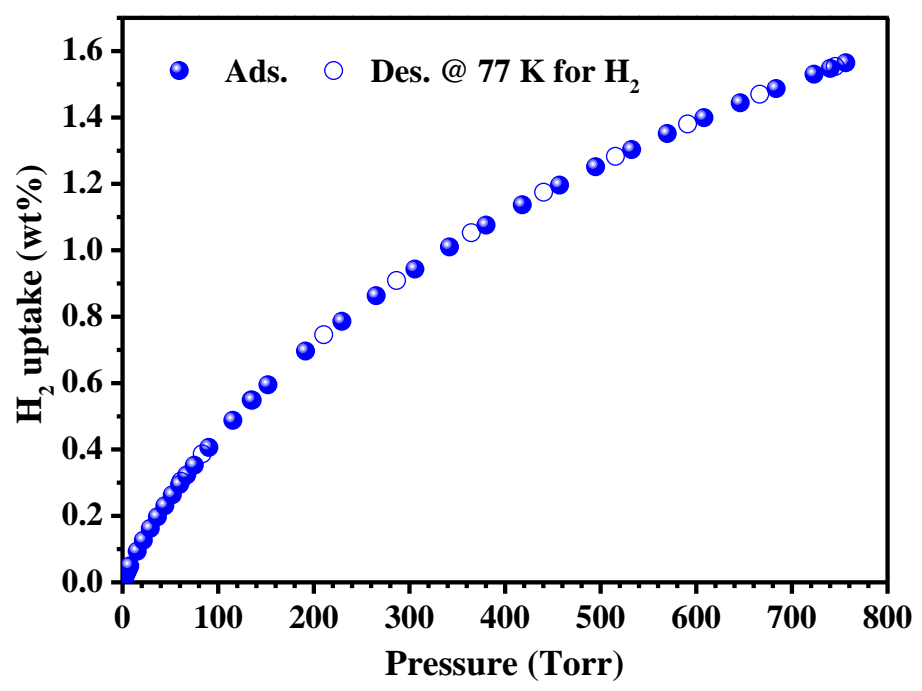

Figure S48. $\mathrm{H}_{2}$ sorption isotherm for $3 \mathrm{~W}-\mathrm{ROD}-2-\mathrm{F}$ measured at $77 \mathrm{~K}$.

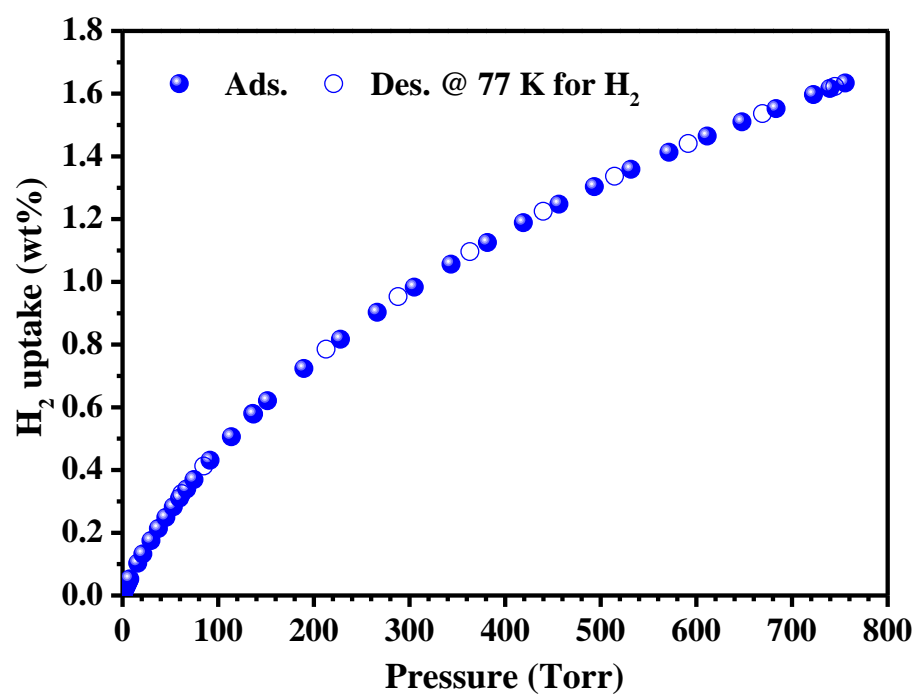

Figure S49. $\mathrm{H}_{2}$ sorption isotherm for $3 \mathrm{~W}-\mathrm{ROD}-2-\mathrm{CH}_{3}$ measured at $77 \mathrm{~K}$. 


\section{Section 7. High Pressure $\mathrm{CH}_{4}$ Adsorption Isotherms and $Q_{\text {st. }}$}

Sample activation. The as-synthesized samples of $3 \mathrm{~W}-\mathrm{ROD}-1$ was exchanged in acetone and n-hexane mixture (4:1) for 3 times during 6 hours, then removed in supercritical $\mathrm{CO}_{2}$ to give activated $3 \mathrm{~W}-\mathrm{ROD}-1$, in the supercritical $\mathrm{CO}_{2}$ process, the sample was exchanged with fresh liquid $\mathrm{CO}_{2}$ for three times, with a duration of 30 min each time. The activated sample was degassed under vacuum for $12 \mathrm{~h}$ at $303 \mathrm{~K}$ and refilled with $\mathrm{N}_{2}$ for storage. $3 \mathrm{~W}-\mathrm{ROD}-2-\mathrm{OH}$ was soaked in acetonitrile for 5 days with acetonitrile refreshing every 8 hours. Then, the acetonitrile exchanged sample of $3 \mathrm{~W}-\mathrm{ROD}-2-\mathrm{OH}$ was activated under vacuum at $303 \mathrm{~K}$ for 4 hours and then heated at $393 \mathrm{~K}$ for 10 hours, 3W-ROD-2-F and 3W-ROD-2- $\mathrm{CH}_{3}$ were soaked in dichloromethane for 24 hours with dichloromethane refreshing every 4 hours, and afterwards, the samples were soaked in n-hexane for 24 hours with n-hexane refreshing every 4 hours. Then, the n-hexane exchanged samples of $3 \mathrm{~W}-\mathrm{ROD}-2-\mathrm{F}$ and $3 \mathrm{~W}-\mathrm{ROD}-2-\mathrm{CH}_{3}$ was activated under vacuum at $303 \mathrm{~K}$ for 4 hours and then heated at $373 \mathrm{~K}$ for 10 hours.

High-pressure adsorption. High-pressure excess methane adsorption isotherms in the range of 0-80 bar were measured with an automatic volumetric sorption apparatus (BELSORP-HP). The measurement temperatures were controlled by a water bath. Ultrahigh-purity He was used to determine the dead space of the sample cell. The adsorption data were corrected to give the final gravimetric excess adsorption isotherm $n_{e x}(P, T)$, by subtracting the background adsorption measured with the empty sample cell using the same test parameters. The total adsorption, which represents the real gas-storage performance of the porous material but cannot be directly measured, was calculated by [eq. (4)]:

$$
n_{\text {tot }}(P, T)=n_{e x}(P, T)+\rho_{g a s}(P, T) \times V_{P}
$$

Where $\rho_{\text {gas }}(P, T)$ is the density of bulk methane at pressure $\mathrm{P}$ and temperature $\mathrm{T}$, and $V_{\mathrm{p}}$ is the pore volume of the porous material determined from $\mathrm{N}_{2}$ adsorption isotherm at $77 \mathrm{~K}$. The volumetric isotherms were obtained by multiplying the gravimetric isotherms with the crystallography density of the host framework. Total $\mathrm{CH}_{4}$ adsorption isotherms for 3W-ROD-1 at 273, 283, 288 and $298 \mathrm{~K}$ were fitted with virial equation [eq. (1)]. Total $\mathrm{CH}_{4}$ adsorption isotherms for 3W-ROD-2-OH, 3W-ROD-2-F, and 3W-ROD-2-CH $\mathrm{H}_{3}$ at 273, 283, 288, and $298 \mathrm{~K}$ were fitted with single-site Tóth Model equations [eq.(5)] as follows, where $n$ is the total amount adsorbed in mmol g-1 $P$ is the pressure in $\mathrm{Pa}, n_{L}$ is the saturation capacity in mmol g$~^{-1}, b$ is the parameter in $\mathrm{Pa}^{-1}$, and $t$ is the heterogeneity parameter of adsorbents. The slope of $\ln P$ vs. $1 / T$ for each uptake amount was obtained. The isosteric heats of adsorption $\left(Q_{\mathrm{st}}\right)$ [eq.(2)] were calculated from the temperature-dependent isotherms using the Clausius-Clapeyron equation [eq.(3)].

$$
\frac{n}{n_{L}}=\frac{b P}{\left[1+(b P)^{t}\right]^{1 / t}}
$$



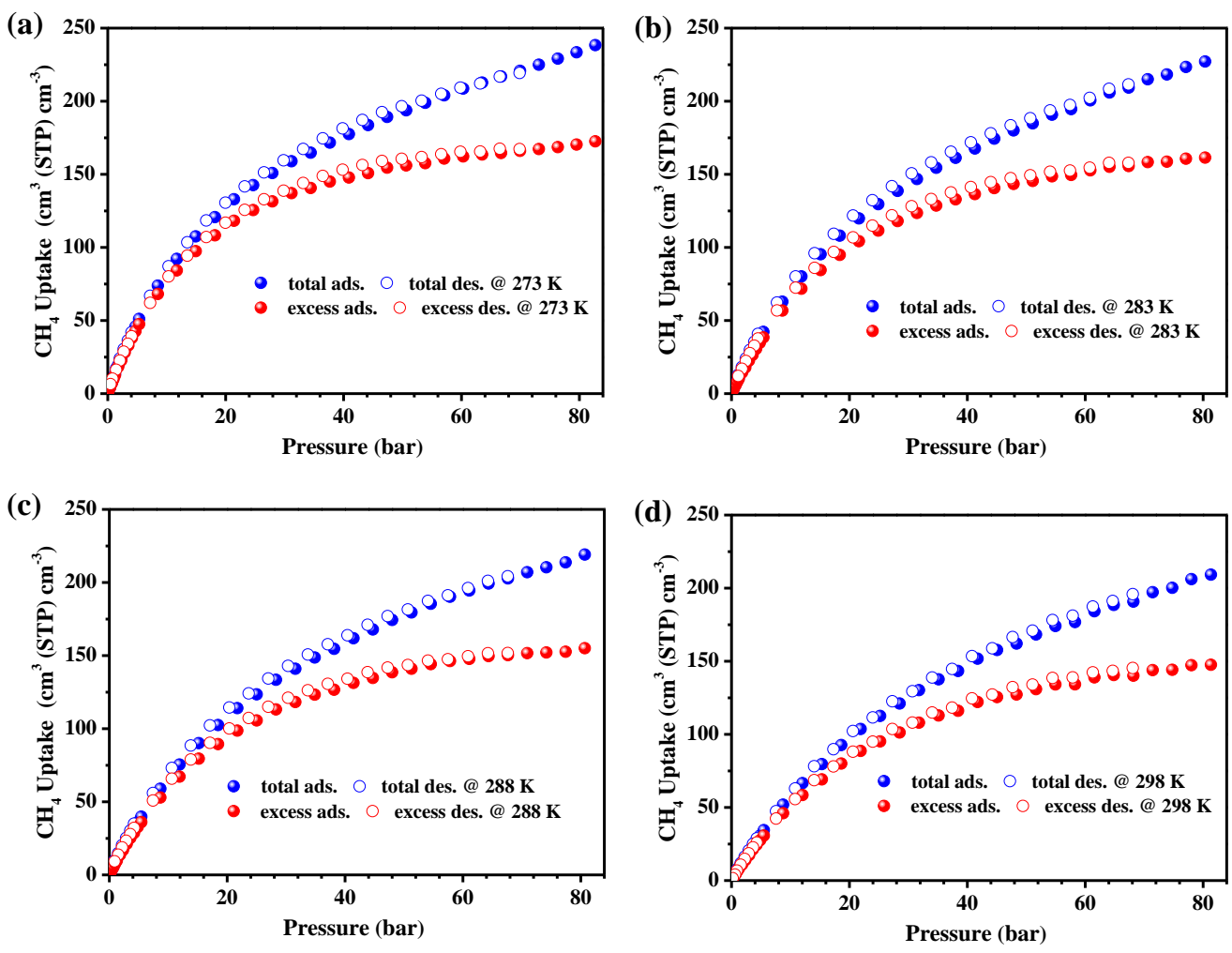

Figure S50. Total and excess methane sorption isotherms for 3W-ROD-1 measured at $273 \mathrm{~K}$ (a), $283 \mathrm{~K}$ (b), $288 \mathrm{~K}$ (c) and $298 \mathrm{~K}$ (d), respectively. 


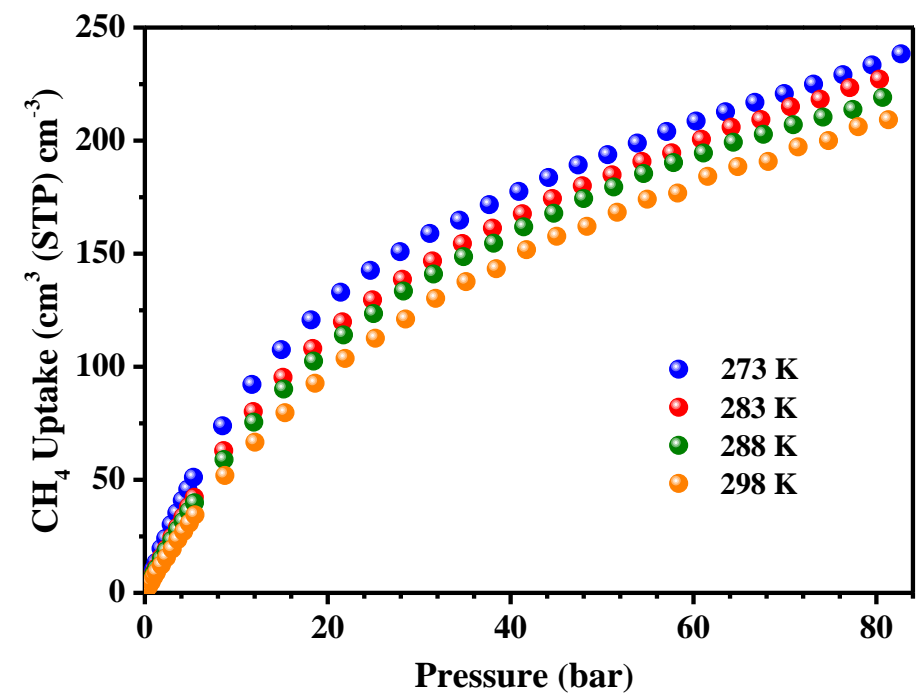

Figure S51. Total methane adsorption isotherms for 3W-ROD-1 measured at 273, 283, 288 and $298 \mathrm{~K}$, respectively.

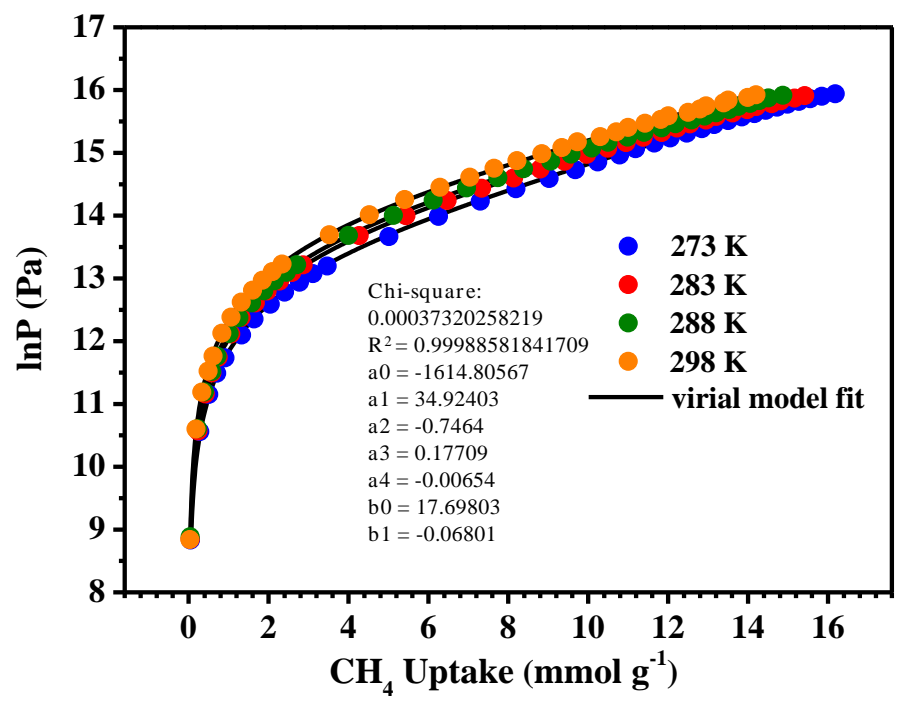

Figure S52. Virial fitting of the temperature-dependent $\mathrm{CH}_{4}$ adsorption isotherms for 3W-ROD-1. 

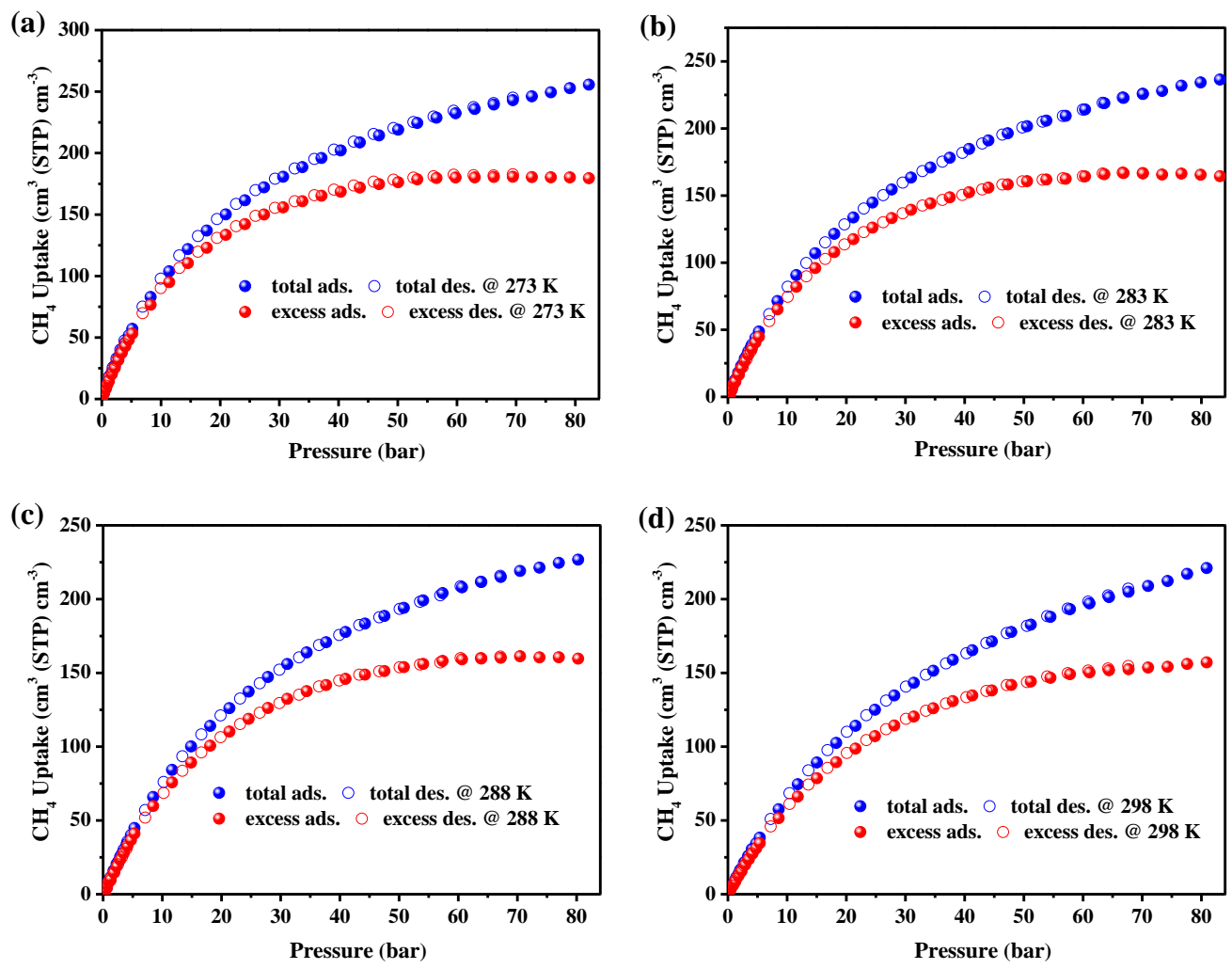

Figure S53. Total and excess methane sorption isotherms for $3 \mathrm{~W}-\mathrm{ROD}-2-\mathrm{OH}$ measured at $273 \mathrm{~K}$ (a), $283 \mathrm{~K}$ (b), $288 \mathrm{~K}$ (c) and $298 \mathrm{~K}$ (d), respectively.

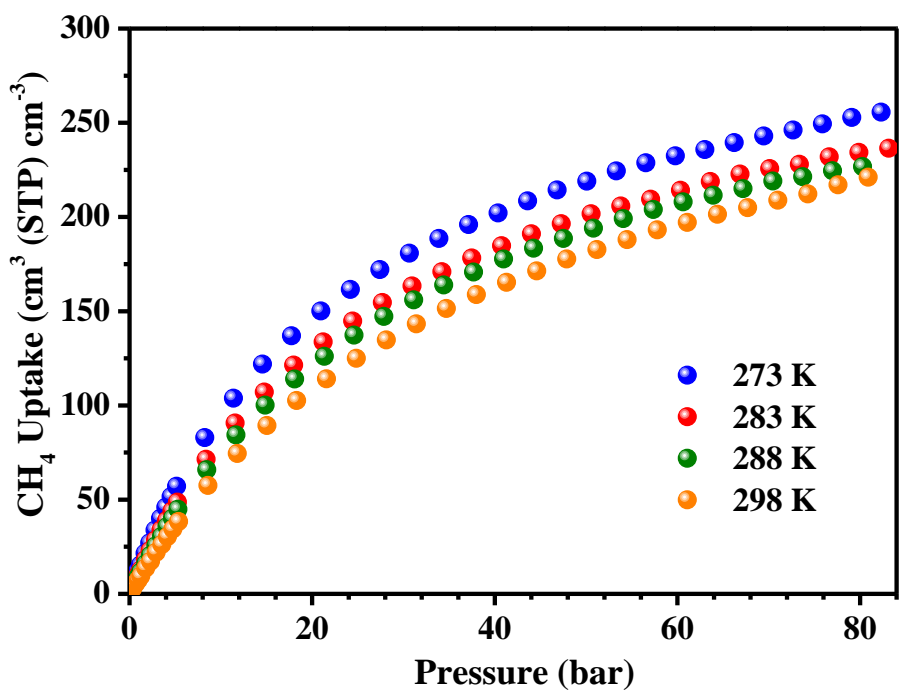

Figure S54. Total methane adsorption isotherms for 3W-ROD-2-OH measured at 273, 283, 288 and $298 \mathrm{~K}$, respectively. 


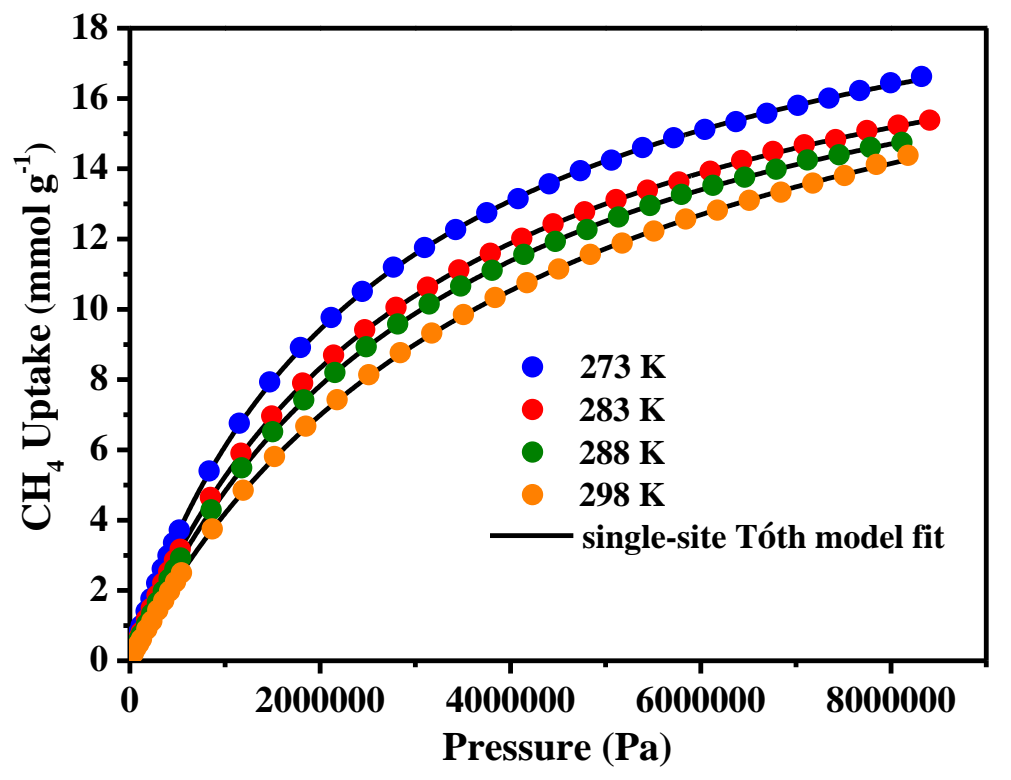

Figure S55. Single-site Tóth Model fitting (lines) of the $3 \mathrm{~W}-\mathrm{ROD}-2-\mathrm{OH} \mathrm{CH}$ adsorption isotherms (points) measured at 273, 283, 288 and $298 \mathrm{~K}$, respectively.

\begin{tabular}{ccccc}
\hline $\mathbf{T}(\mathbf{K})$ & $\mathbf{2 7 3}$ & $\mathbf{2 8 3}$ & $\mathbf{2 8 8}$ & $\mathbf{2 9 8}$ \\
\hline$n_{L}$ & $22.65132 \pm 0.14239$ & $21.45262 \pm 0.13122$ & $20.80042 \pm 0.12082$ & $21.90225 \pm 0.27043$ \\
$b$ & $3.97235 \mathrm{E}-7 \pm 1.82897 \mathrm{E}-9$ & $3.35535 \mathrm{E}-7 \pm 1.31425 \mathrm{E}-9$ & $3.01653 \mathrm{E}-7 \pm 1.02929 \mathrm{E}-9$ & $2.40889 \mathrm{E}-7 \pm 1.57335 \mathrm{E}-9$ \\
$t$ & $0.91727 \pm 0.00896$ & $0.95214 \pm 0.0088$ & $1.00015 \pm 0.00865$ & $0.97151 \pm 0.01547$ \\
$R^{2}$ & 0.99997 & 0.99998 & 0.99998 & 0.99996 \\
\hline
\end{tabular}

Table S2. The obtained single-site Tóth model fitting parameters for $\mathrm{CH}_{4}$ adsorption isotherms of $3 \mathrm{~W}-\mathrm{ROD}-2-\mathrm{OH}$. 
(a)
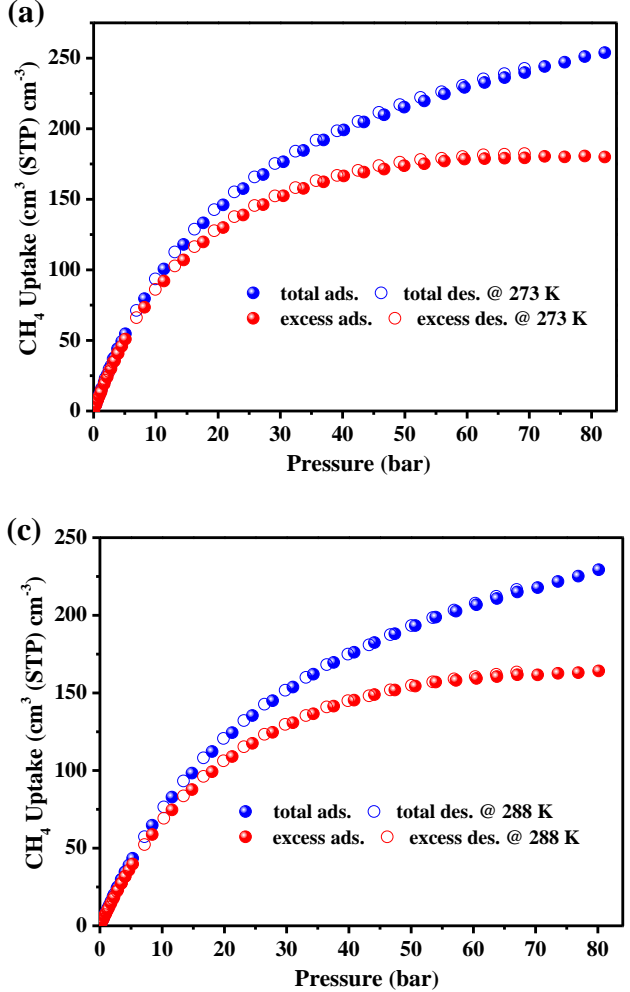

(b)
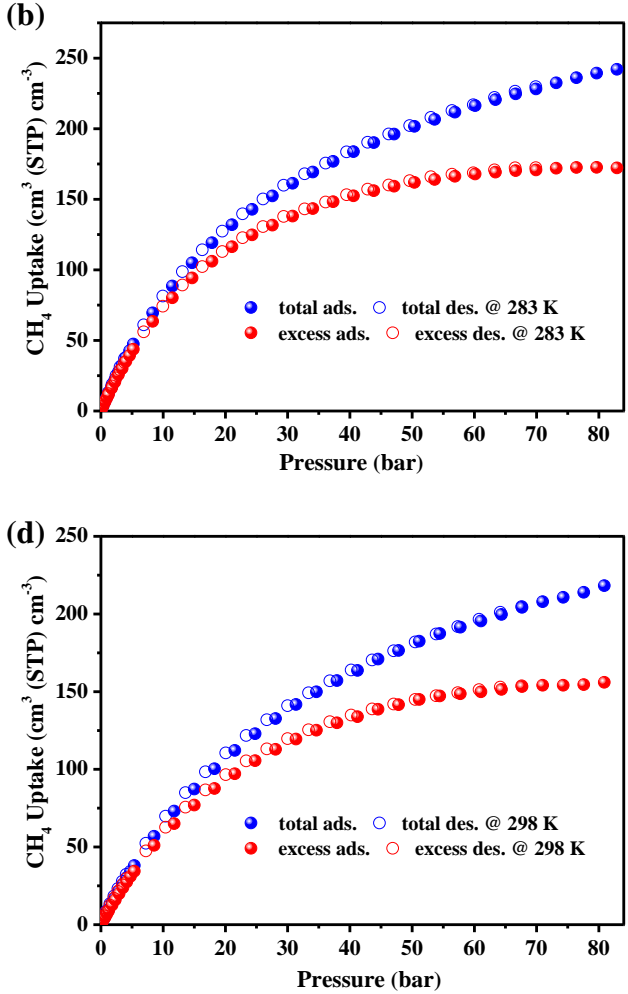

Figure S56. Total and excess methane sorption isotherms of 3W-ROD-2-F measured at $273 \mathrm{~K}$ (a), $283 \mathrm{~K}$ (b), $288 \mathrm{~K}$ (c) and $298 \mathrm{~K}$ (d), respectively.

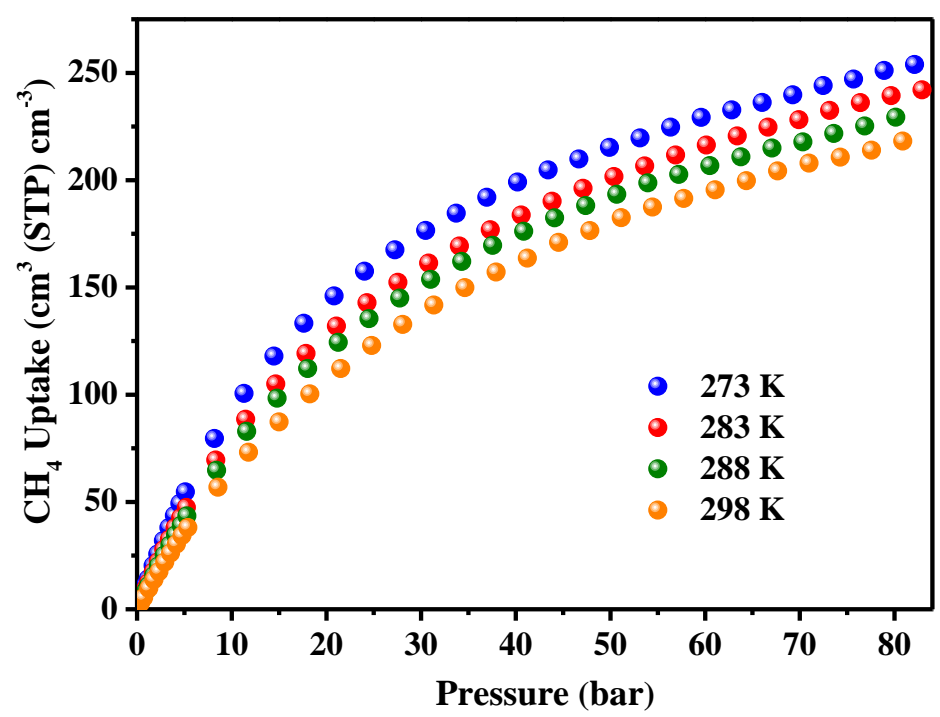

Figure S57. Total methane adsorption isotherms for 3W-ROD-2-F measured at 273, 283, 288 and $298 \mathrm{~K}$, respectively. 


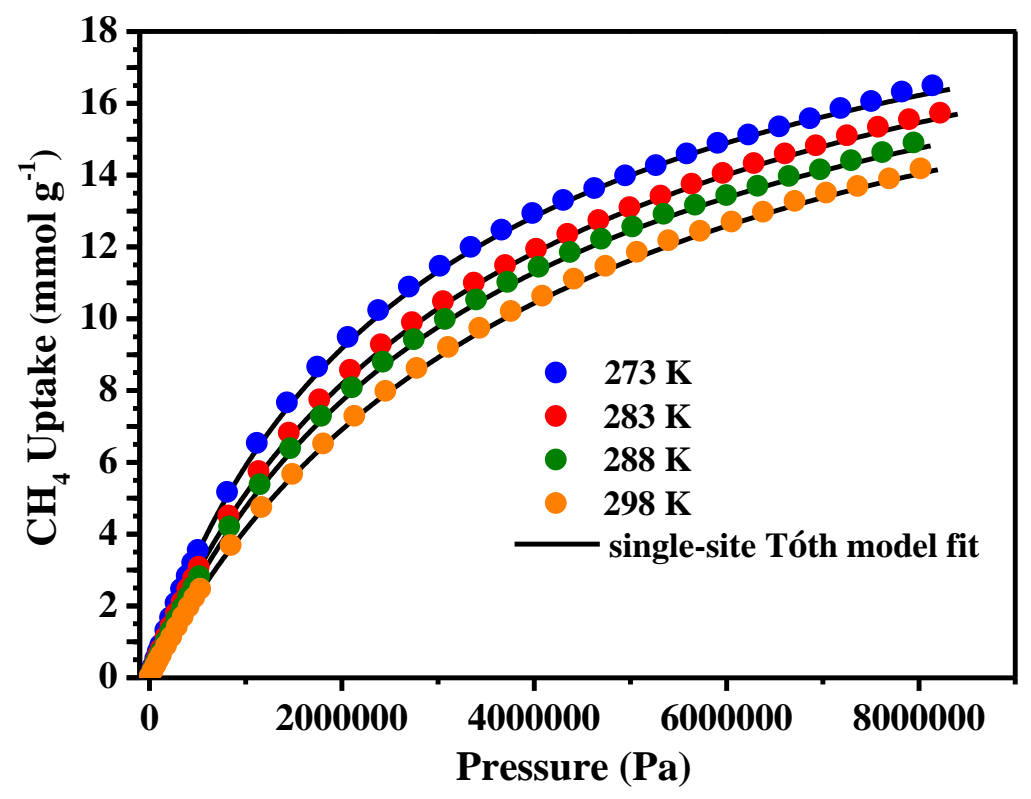

Figure S58. Single-site Tóth Model fitting (lines) of the $3 \mathrm{~W}-\mathrm{ROD}-2-\mathrm{F} \mathrm{CH}_{4}$ adsorption isotherms (points) measured at 273, 283, 288 and $298 \mathrm{~K}$.

\begin{tabular}{ccccc}
\hline $\boldsymbol{T}(\boldsymbol{K})$ & $\mathbf{2 7 3}$ & $\mathbf{2 8 3}$ & $\mathbf{2 8 8}$ & $\mathbf{2 9 8}$ \\
\hline$n_{L}$ & $22.9663 \pm 0.25204$ & $23.96811 \pm 0.27461$ & $21.68171 \pm 0.17319$ & $21.63265 \pm 0.18068$ \\
$b$ & $3.76704 \mathrm{E}-7 \pm 2.80466 \mathrm{E}-9$ & $3.03372 \mathrm{E}-7 \pm 1.89532 \mathrm{E}-9$ & $2.87367 \mathrm{E}-7 \pm 1.27326 \mathrm{E}-9$ & $2.38472 \mathrm{E}-7 \pm 1.05803 \mathrm{E}-9$ \\
$t$ & $0.90333 \pm 0.01472$ & $0.86812 \pm 0.01278$ & $0.96204 \pm 0.01065$ & $0.9828 \pm 0.01066$ \\
$R^{2}$ & 0.99993 & 0.99995 & 0.99997 & 0.99998 \\
\hline
\end{tabular}

Table S3. The obtained Single-site Tóth Model fitting parameters for $\mathrm{CH}_{4}$ adsorption isotherms of $3 \mathrm{~W}-\mathrm{ROD}-2-\mathrm{F}$. 

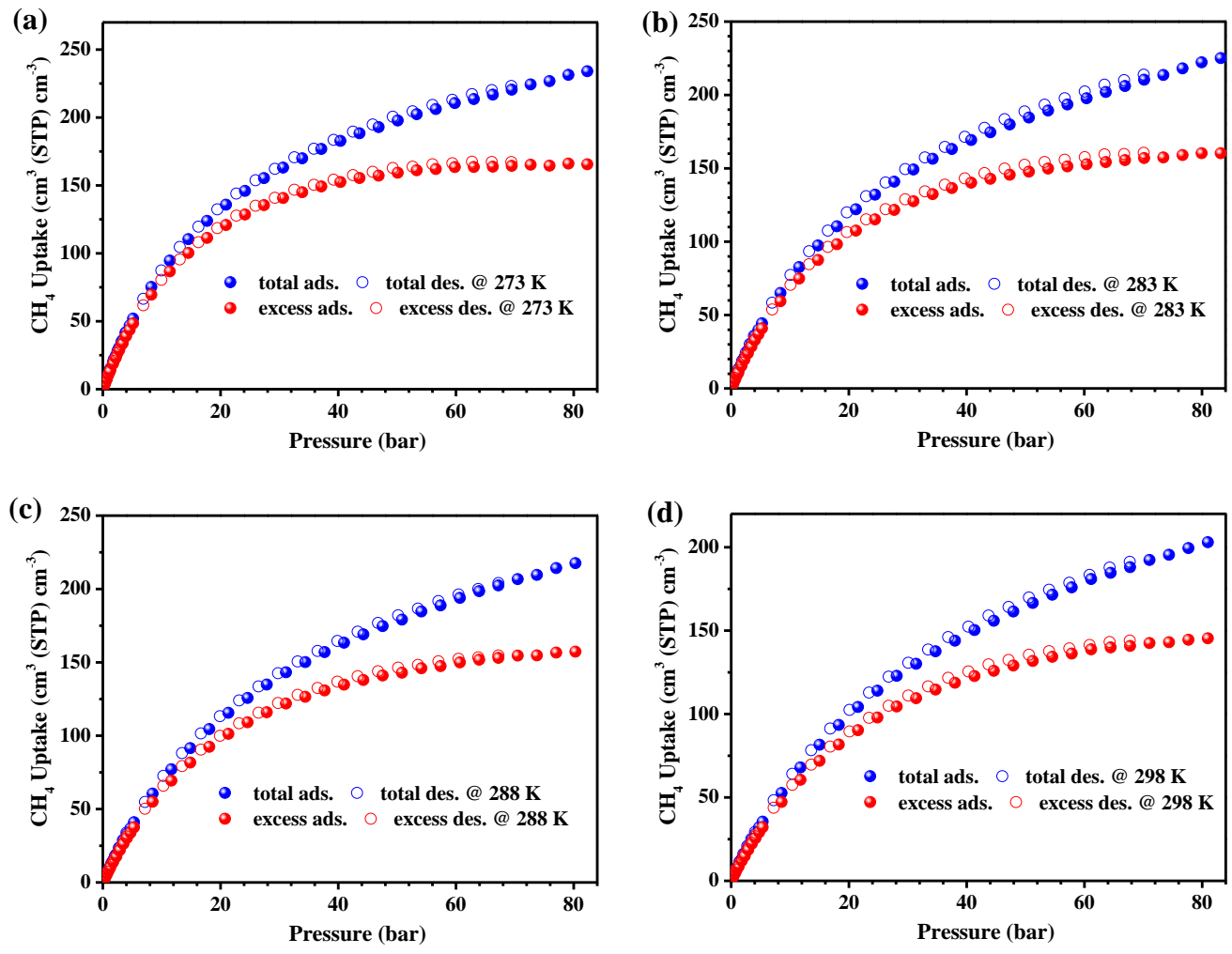

Figure S59. Total and excess methane sorption isotherms of $3 \mathrm{~W}-\mathrm{ROD}-2-\mathrm{CH}_{3}$ measured at $273 \mathrm{~K}$ (a), $283 \mathrm{~K}$ (b), $288 \mathrm{~K}$ (c), and $298 \mathrm{~K}$ (d), respectively.

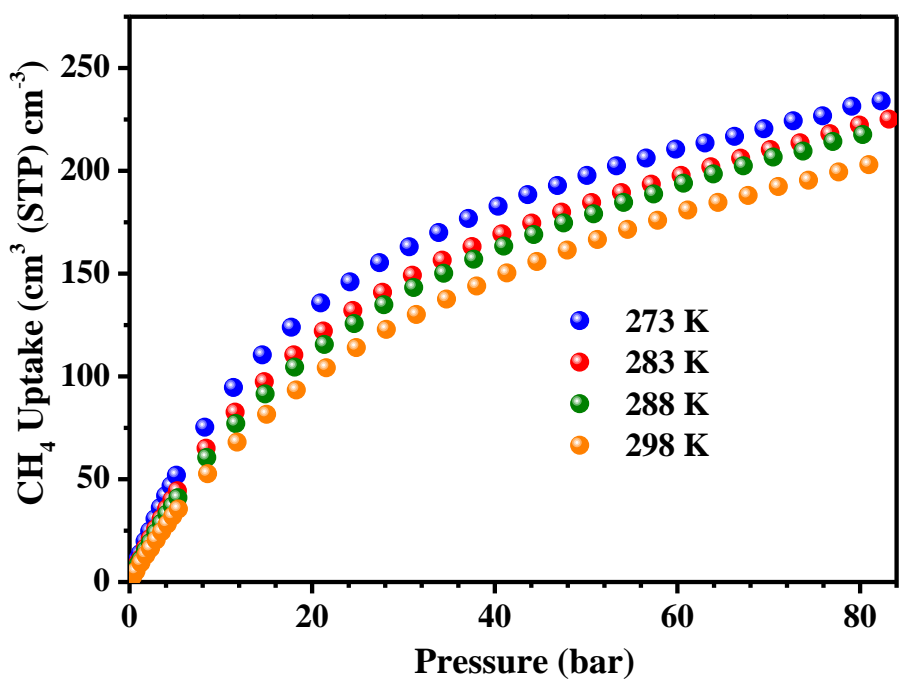

Figure S60. Total methane adsorption isotherms for $3 \mathrm{~W}-\mathrm{ROD}-2-\mathrm{CH}_{3}$ measured at 273, 283, 288 and $298 \mathrm{~K}$, respectively. 


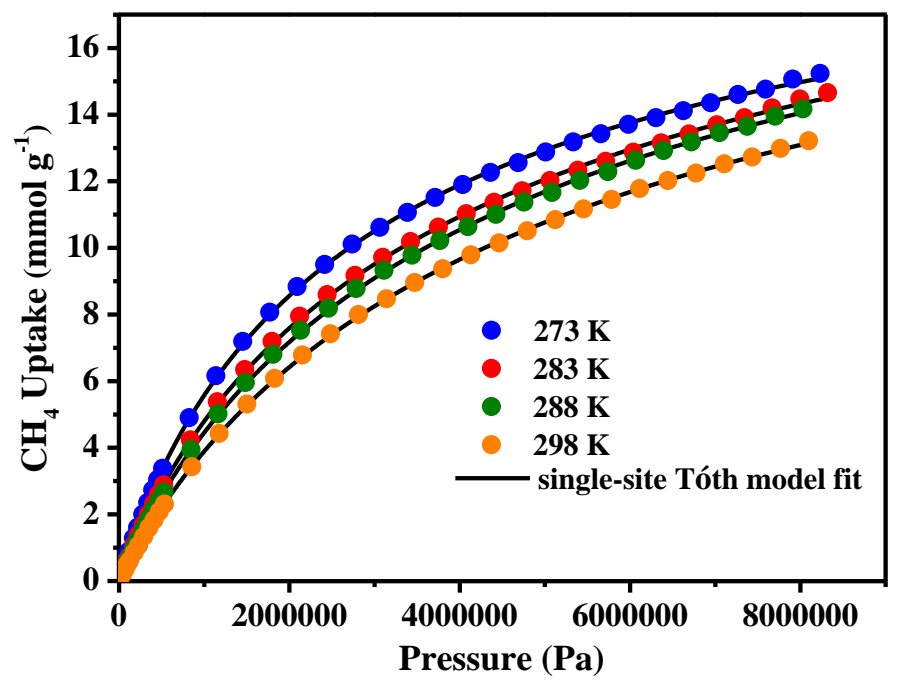

Figure S61. Single-site Tóth Model fitting (lines) of the $3 \mathrm{~W}-\mathrm{ROD}-2-\mathrm{CH}_{3} \mathrm{CH}_{4}$ adsorption isotherms (points) measured at 273, 283, 288 and $298 \mathrm{~K}$, respectively.

\begin{tabular}{ccccc}
\hline $\boldsymbol{T}(\boldsymbol{K})$ & $\mathbf{2 7 3}$ & $\mathbf{2 8 3}$ & $\mathbf{2 8 8}$ & $\mathbf{2 9 8}$ \\
\hline$n_{L}$ & $21.57348 \pm 0.31558$ & $23.10379 \pm 0.57532$ & $23.56225 \pm 0.51643$ & $22.16045 \pm 0.34433$ \\
$b$ & $4.00872 \mathrm{E}-7 \pm 3.98887 \mathrm{E}-9$ & $3.09553 \mathrm{E}-7 \pm 4.04849 \mathrm{E}-9$ & $2.70412 \mathrm{E}-7 \pm 2.9485 \mathrm{E}-9$ & $2.34486 \mathrm{E}-7 \pm 1.85198 \mathrm{E}-9$ \\
$t$ & $0.85776 \pm 0.01785$ & $0.8157 \pm 0.02436$ & $0.82235 \pm 0.02019$ & $0.86772 \pm 0.01508$ \\
$R^{2}$ & 0.99988 & 0.99981 & 0.99989 & 0.99995 \\
\hline
\end{tabular}

Table S4. The obtained Single-site Tóth Model fitting parameters for $\mathrm{CH}_{4}$ adsorption isotherms of $3 \mathrm{~W}-\mathrm{ROD}-2-\mathrm{CH}_{3}$. 


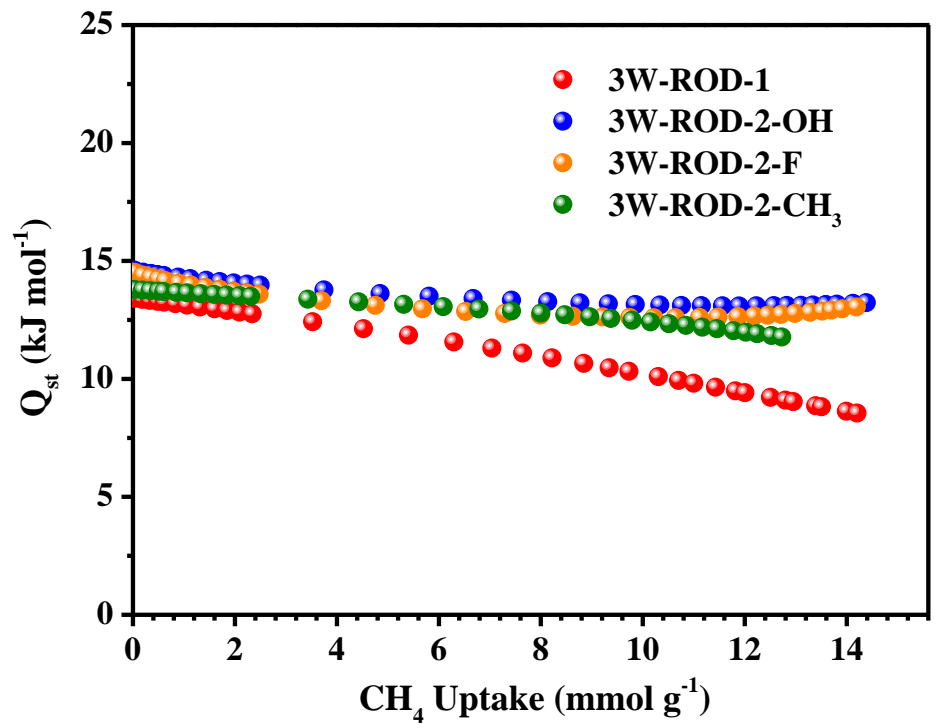

Figure S62. Calculated $\mathrm{CH}_{4} Q_{\mathrm{st}}$ of $3 \mathrm{~W}-\mathrm{ROD}-1$ and $3 \mathrm{~W}-\mathrm{ROD}-2-\mathrm{X}(\mathrm{X}=-\mathrm{OH},-\mathrm{F}$ and $-\mathrm{CH}_{3}$ ). 
Table S5. Total $\mathrm{CH}_{4}$ volumetric uptake and working capacity for $3 \mathrm{~W}-\mathrm{ROD}-1$ and $3 \mathrm{~W}-\mathrm{ROD}-2-\mathrm{X}$ in comparison with some promising MOFs at $298 \mathrm{~K}$.

\begin{tabular}{|c|c|c|c|c|c|c|c|c|}
\hline MOFs & $\mathrm{OMS}^{\mathrm{a}}$ & $\begin{array}{c}\mathrm{BET}^{\mathrm{b}} \\
\left(\mathrm{m}^{2} \mathrm{~g}^{-1}\right)\end{array}$ & $\begin{array}{c}D_{\mathrm{c}}^{\mathrm{c}} \\
\left(\mathrm{g} \mathrm{cm}^{-3}\right)\end{array}$ & $\begin{array}{c}\mathrm{V}_{\mathrm{p}}^{\mathrm{d}} \\
\left(\mathrm{cm}^{3} \mathrm{~g}^{-1}\right)\end{array}$ & $\begin{array}{c}\text { Total uptake at } \\
5 / 35 / 65 / 80 \text { bar } \\
\left(\mathrm{cm}^{3} \mathrm{~cm}^{-3}\right)\end{array}$ & $\begin{array}{l}\text { Working capacity } \\
\text { at } 35 / 65 / 80 \text { bar } \\
\left(\mathrm{cm}^{3} \mathrm{~cm}^{-3}\right)\end{array}$ & $\begin{array}{c}Q_{\mathrm{st}} \\
\left(\mathrm{kJ} \mathrm{mol}^{-1}\right)\end{array}$ & Ref. \\
\hline Ni-MOF-74 & $\mathrm{Ni}$ & 1350 & 1.206 & 0.51 & $115 / 230 / 260 / 267$ & $115 / 145 / 152$ & 21.4 & 5,23 \\
\hline Co(bdp) & Co & $2911^{\mathrm{e}}$ & 0.77 & 0.93 & $6^{\mathrm{f}} / 161 / 203 / \mathrm{NA}$ & 155/197/NA & 13.0 & 4 \\
\hline HKUST-1 & $\mathrm{Cu}$ & 1850 & 0.883 & 0.78 & $72 / 225 / 262 / 272$ & $153 / 190 / 200$ & 17.0 & 5,23 \\
\hline PCN-14 & $\mathrm{Cu}$ & 2000 & 0.819 & 0.83 & $72 / 195 / 230 / 250$ & $123 / 157 / 178$ & 18.7 & 5,23 \\
\hline MOF-519 & NA & 2400 & 0.953 & 0.938 & 49/200/259/279 & $151 / 210 / 230$ & 14.6 & 24 \\
\hline MOF-905 & NA & 3490 & 0.549 & 1.34 & $25 / 145 / 206 / 228$ & $120 / 181 / 203$ & 11.7 & 25 \\
\hline MOF-950 & NA & 3440 & 0.517 & 1.30 & $36 / 145 / \mathrm{NA} / 209$ & 109/NA/174 & 11.9 & 25 \\
\hline MOF-205 & NA & 4460 & 0.380 & 2.16 & 19/120/NA/205 & 101/NA/186 & 10.6 & 26 \\
\hline MOF-210 & NA & 6240 & 0.250 & 3.6 & 12/82/NA/166 & 70/NA/154 & NA & 26 \\
\hline MOF-177 & NA & 4500 & 0.427 & 1.89 & 20/122/NA/205 & 102/NA/185 & 9.9 & 26 \\
\hline MOF-5 & NA & 3320 & 0.605 & 1.38 & 22/126/NA/198 & 104/NA/176 & 10.0 & 26 \\
\hline NJU-Bai43 & $\mathrm{Cu}$ & 3090 & 0.639 & 1.22 & 56/202/254/NA & 146/198/NA & 14.45 & 27 \\
\hline UTSA-76a & $\mathrm{Cu}$ & 2820 & 0.699 & 1.09 & 60/211/257/NA & 151/197/NA & 15.44 & 28 \\
\hline UTSA-110a & $\mathrm{Cu}$ & 3241 & 0.600 & 1.263 & $51^{\mathrm{f}} / \mathrm{NA} / 241 / \mathrm{NA}$ & NA/190/NA & 14.5 & 29 \\
\hline Al-soc-MOF-1 & $\mathrm{Al}$ & 5585 & 0.378 & 2.3 & $21 / 127 / 197 / 222$ & $106 / 176 / 201$ & 11 & 30 \\
\hline PCN-46a & $\mathrm{Cu}$ & 3224 & 0.619 & 1.243 & $43 / 172 / 233 / 246$ & $129 / 190 / 203$ & 14.2 & 31 \\
\hline MFM-115a & $\mathrm{Cu}$ & 3394 & 0.611 & 1.38 & $48 / 186 / 238 / 256$ & $138 / 191 / 208$ & 16.3 & 32 \\
\hline MFM-132a & $\mathrm{Cu}$ & 2466 & 0.650 & 1.06 & $51 / 162 / 201 / 213$ & $109 / 150 / 162$ & 15.7 & 32 \\
\hline pbz-MOF-1 & $\mathrm{Zr}$ & 2415 & 0.663 & 0.99 & $30 / 140 / \mathrm{NA} / 210$ & 110/NA/180 & NA & 33 \\
\hline FDM-8 & NA & 3643 & 0.563 & 1.543 & 22/NA/NA/215 & NA/NA/193 & 10.4 & 34 \\
\hline MAF-38 & NA & 2022 & 0.761 & 0.808 & $76 / 226 / 263 / 273$ & $150 / 187 / 197$ & 21.6 & 35 \\
\hline ST-2 & NA & 5172 & 0.366 & 2.44 & 19/NA/181/206 & NA/162/187 & 9.2 & 36 \\
\hline Cu-tbo-MOF-5 & $\mathrm{Cu}$ & 3971 & 0.595 & 1.12 & $41 / 151 / 199 / 216$ & $110 / 158 / 175$ & 20.4 & 37 \\
\hline NU-1501-A1 ${ }^{\mathrm{g}}$ & $\mathrm{Al}$ & 7310 & 0.283 & 2.91 & 16/NA/163/190 & NA/147/174 & 9.7 & 38 \\
\hline 3W-ROD-1 & NA & 2742 & 0.657 & 1.11 & $31 / 137 / 189 / 208$ & $106 / 158 / 177$ & 13.41 & This work \\
\hline $3 \mathrm{~W}-\mathrm{ROD}-2-\mathrm{OH}$ & NA & 2552 & 0.670 & 1.11 & $36 / 152 / 202 / 220$ & $116 / 166 / 184$ & 14.60 & This work \\
\hline 3W-ROD-2-F & NA & 2482 & 0.670 & 1.08 & $36 / 150 / 201 / 217$ & $114 / 165 / 181$ & 14.51 & This work \\
\hline $3 \mathrm{~W}-\mathrm{ROD}-2-\mathrm{CH}_{3}$ & NA & 2315 & 0.669 & 1.00 & $34 / 138 / 185 / 202$ & $104 / 151 / 168$ & 13.79 & This work \\
\hline
\end{tabular}

a: open metal sites. b: surface areas calculated from $\mathrm{N}_{2}$ isotherms at $77 \mathrm{~K}$. c: framework density. d: pore volume. e: Langmuir surface area. f: data from 5.8 bar. g: measured at $296 \mathrm{~K}$. $Q_{\text {st: }}$ coverage-dependent adsorption enthalpies at near-zero coverage. NA: not available. 


\section{Section 8. Computational Methods}

Monte Carlo molecular dynamics (MCMD) ${ }^{39}$ software was used to simulate the uptake of $\mathrm{CH}_{4}$ at $273 \mathrm{~K}$ and $298 \mathrm{~K}$ from 0 to 1 or $80 \mathrm{~atm}$. Grand Canonical Monte Carlo (GCMC) calculations were used, which restrains the chemical potential, volume, and temperature to be constant while allowing particle number to fluctuate. The chemical potential is determined from appropriate equations of state ${ }^{40}$ for the gas simulated using the input pressure and temperature, and volume is given by crystallographic unit cell parameters. Empirical simulations were performed using a unit cell. The force field utilized was a combination of Lennard-Jones and Ewald electrostatics. Parameters for Lennard-Jones repulsion dispersion were taken from the Universal Force Field. ${ }^{41}$ Partial charges for each atom were obtained from quantum mechanical calculations based on fragments taken from the crystal structure using NWChem. ${ }^{42}$ Charges and fragments are shown in Figures S63-S65. Parameters for $\mathrm{CH}_{4}$ were those of the TraPPE $\mathrm{CH}_{4}$ model. ${ }^{43}$ The isosteric heat $\left(Q_{\mathrm{st}}\right)$ was also calculated in these GCMC simulations from statistical mechanical fluctuations of the number of particles, $\mathrm{N}$, and potential energy, $\mathrm{U}$, via [eq.(6)],

$$
Q_{\mathrm{st}}=-\frac{\langle N U\rangle-\langle N\rangle\langle U\rangle}{\left\langle N^{2}\right\rangle-\langle N\rangle^{2}}+k T
$$

where $\mathrm{T}$ is the temperature and $\mathrm{k}$ is Boltzmann's constant. The simulated isotherms and corresponding $Q_{\mathrm{st}}$ are shown in Figures S66-S68.

Binding site calculates were performed via simulated annealing, whereby a single $\mathrm{CH}_{4}$ molecule was inserted into the unit cell and modeled with the force field as described above with MCMD. Temperature was annealed from $600 \mathrm{~K}$ to approximately $1 \mathrm{~K}$ over a series of Monte Carlo translation/rotation trial moves to minimize binding energy. The temperature was reduced by an exponential scale with a factor of 0.9995. The resulting binding site is shown in Figures S69-S71. 
(a)

In 2.586444375

O1 -1.399060852

O2 -0.591772390

O3 -0.600537934

O4 -0.843343136

O5 - 0.760748865

$\mathrm{N}-0.67979985$

C1 0.8886235

C2 0.0022383

C3 -0.122058245

C4 0.8200132

C5 -0.078139237

C6 -0.256846898

C7 0.52682413

C8 -0.129463085

C9 0.07247933

C10 - 0.3426879468

C11 - 0.5577897683

C12 0.517091

C13 -0.1445382895

H1 0.415145

H2 0.3302678

H3 0.167449083

H4 0.1226725

H5 0.1032546

H6 0.1542294

H7 0.191586

H8 0.231328

(b) In 2.586444375

O1 -0.591772390383586

O3-0.843343136377173

O4-0.760748865444527

O5-0.679799850646348

N 0.8886235

C1 0.002238333333333

C2 -0.122058245674975

C3 0.8200132

C4 - 0.078139237939134

C5 -0.25684689894031

C6 0.526824133333333

C7 -0.129463085518771

C8 -0.131735157228524

C9 0.072479333333333

C10 -0.326005997485483

H1 0.3309694

H2 0.167544416666667

H3 0.1239785

H4 0.103254666666667

H5 0.1544806

H6 0.191586
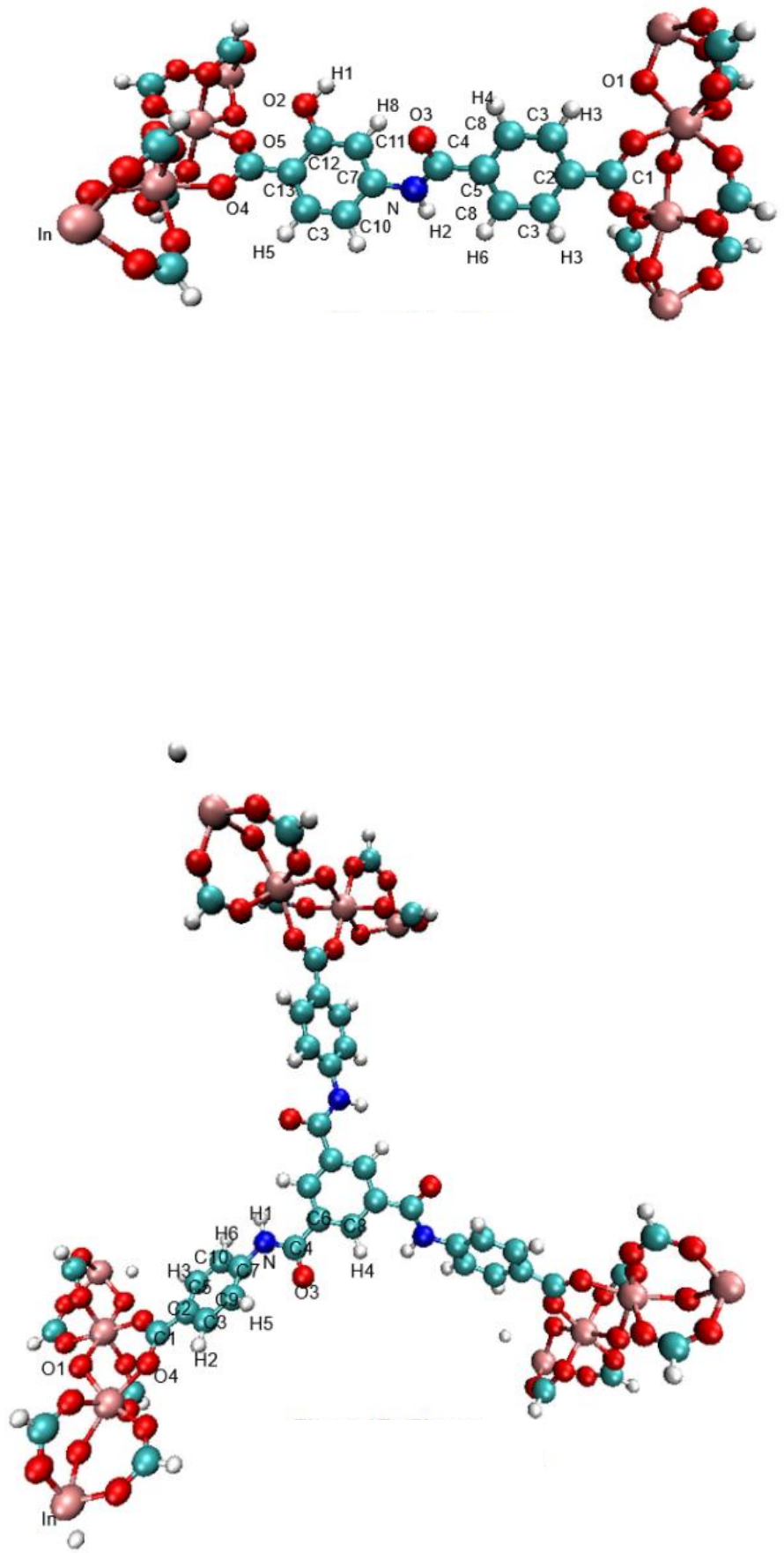

Figure S63. Charge parameterization of 3W-ROD-2-OH by NWChem using 6-31G* for all atoms except In, which used LANL2DZ ECP. 
(a) In 2.589658375

$\mathrm{Cl}-0.233081613787673$

O1 -1.40198366488262

O3 -0.600738186525209

O4 -0.843412850206648

O5 -0.781654893421028

$\mathrm{N}-0.681118403626353$

C1 0.88552725

C2 0.002314933333333

C3 -0.123706688741776

C4 0.8192582

C5 -0.074776473709429

C6 - 0.257299613616653

C7 0.519592133333333

C8 -0.131735157228524

C9 0.072479333333333

C10 -0.326005997485483

C11 - 0.462778454962473

C12 0.426461

C13 -0.115111198518171

H1 0.330397

H2 0.15787125

H3 0.1220675

H4 0.1032546666666667

H5 0.153931

H6 0.191586

H7 0.20809

(b)

In 2.589658375

O1 -1.40198366488262

O3 -0.600738186525209

O4 - 0.843412850206648

O5 -0.781654893421028

$\mathrm{N}-0.681118403626353$

C1 0.88552725

C2 0.002314933333333

C3 -0.123706688741776

C4 0.8192582

C5 -0.074776473709429

C6 -0.257299613616653

C7 0.519592133333333

C8 0.072479333333333

C9 -0.342687946835276

C10 -0.557789768357453

H1 0.3302678

H2 0.167449083333333

H3 0.1226725

H4 0.103254666666667

H5 0.1542294

H6 0.191586
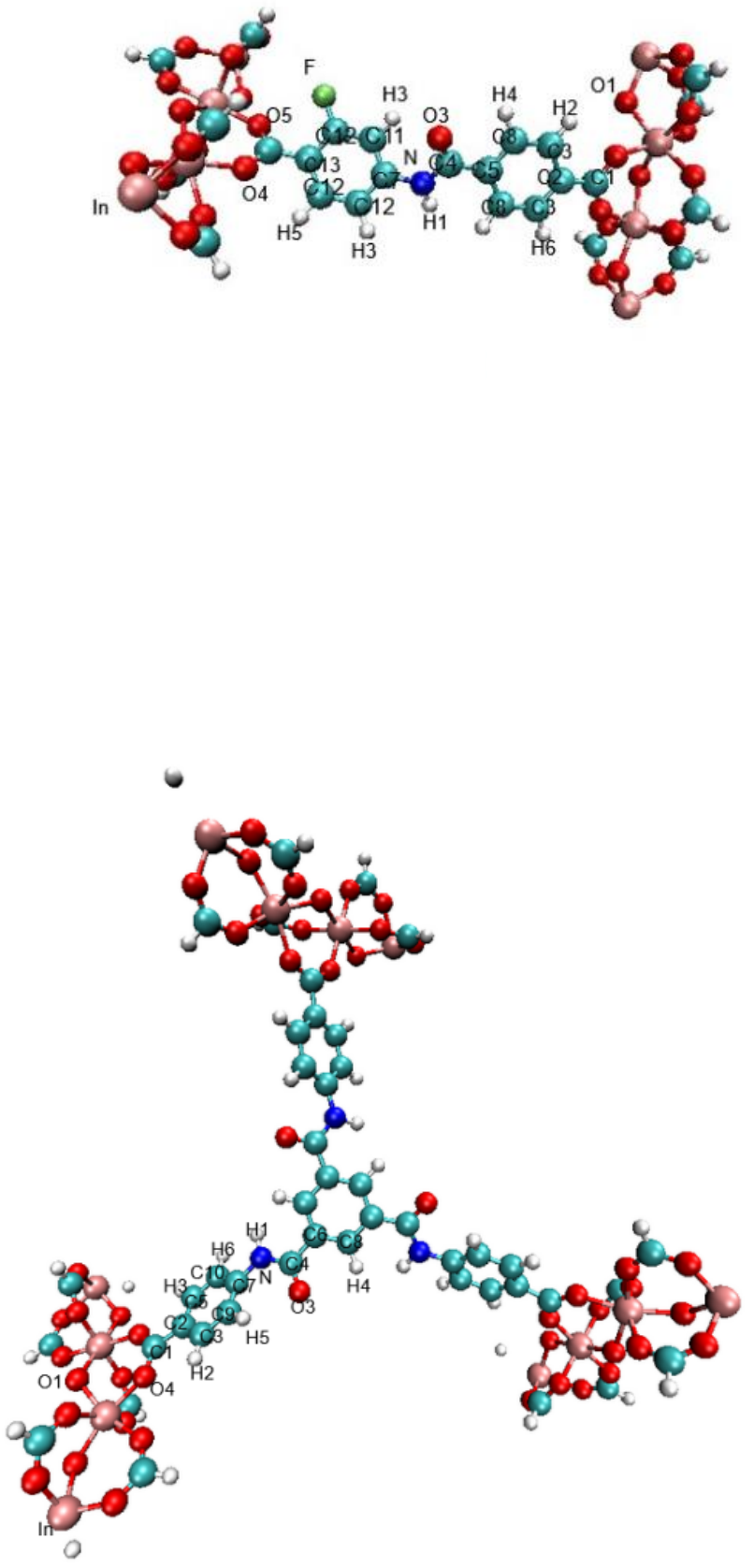

Figure S64. Charge parameterization of 3W-ROD-2-F by NWChem using 6-31G* for all atoms except In, which used LANL2DZ ECP. 
(a) In 2.584696125

O1 -1.39260057373696

O3 -0.596949583599465

O4 -0.835360253655331

O5 -0.752427706476859

$\mathrm{N}-0.677224019137613$

C0 -0.140063410790594

C1 0.87887925

C2 0.001410733333333

C3 -0.123210209619999

C4 0.818666

C5 -0.067997299548197

C6 - 0.255383737340592

C7 0.522485733333333

C8 - 0.132645079801203

C9 0.072479333333333

C10 -0.328702109690159

C11 - 0.496172969691025

C12 0.29113

C13 -0.124350963655333

H1 0.3302814

H2 0.165498583333333

H3 0.123522

H4 0.103254666666667

H5 0.15303

H6 0.191586

H7 0.062068

H8 0.077495

H9 0.00661

(b) In 2.584696125

O1 -1.39260057373696

O3 -0.596949583599465

O4 - 0.835360253655331

O5 -0.752427706476859

N -0.677224019137613

C1 0.87887925

C2 0.001410733333333

C3 -0.123210209619999

C4 0.818666

C5 -0.067997299548197

C6 - 0.255383737340592

C7 0.522485733333333

C8 - 0.132645079801203

C9 0.072479333333333

C10 -0.328702109690159

H1 0.3302814

H2 0.165498583333333

H3 0.123522

H4 0.103254666666667

H5 0.15303

H6 0.191586
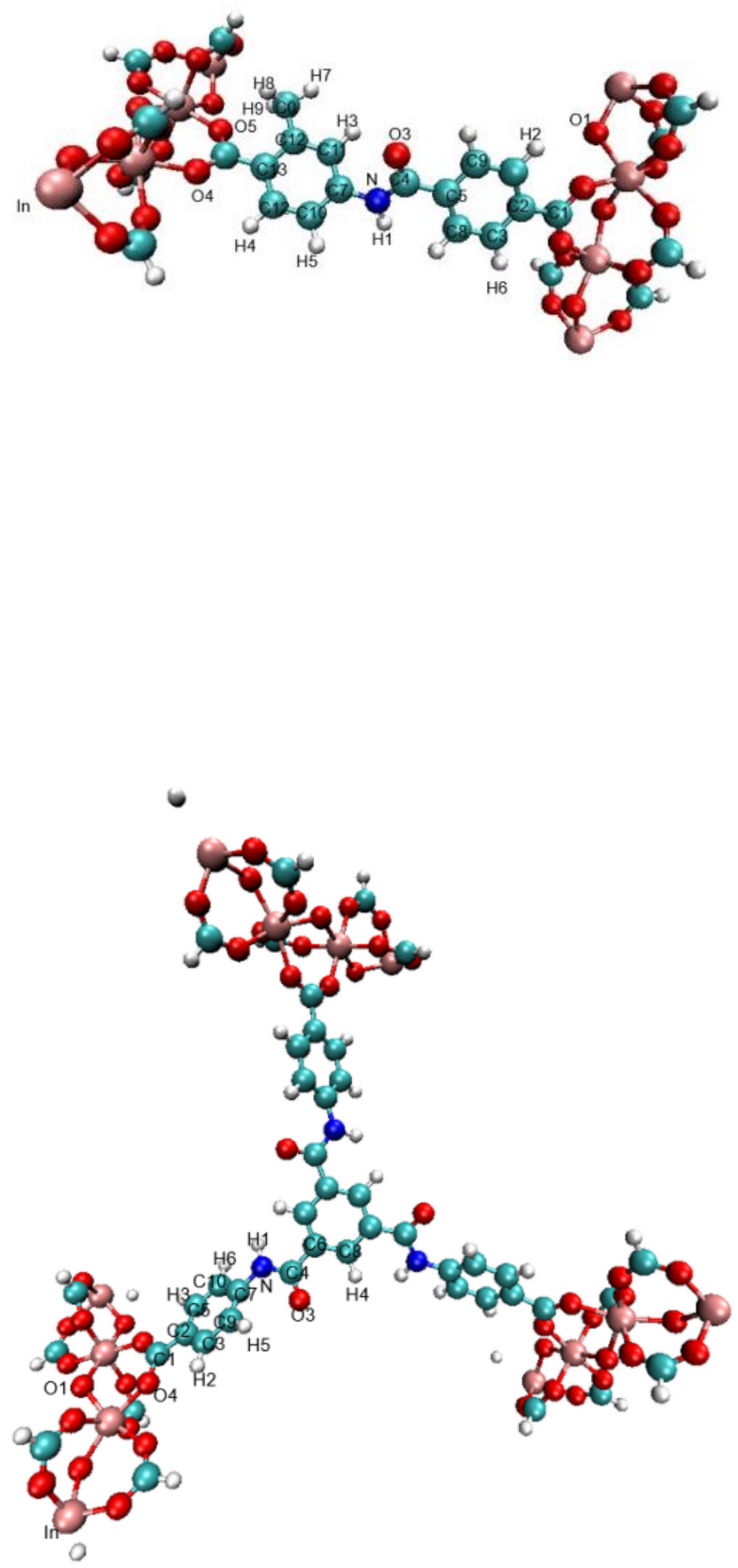

Figure S65. Charge parameterization of 3W-ROD-2- $\mathrm{CH}_{3}$ by NWChem using 6-31G* for all atoms except In, which used LANL2DZ ECP. 

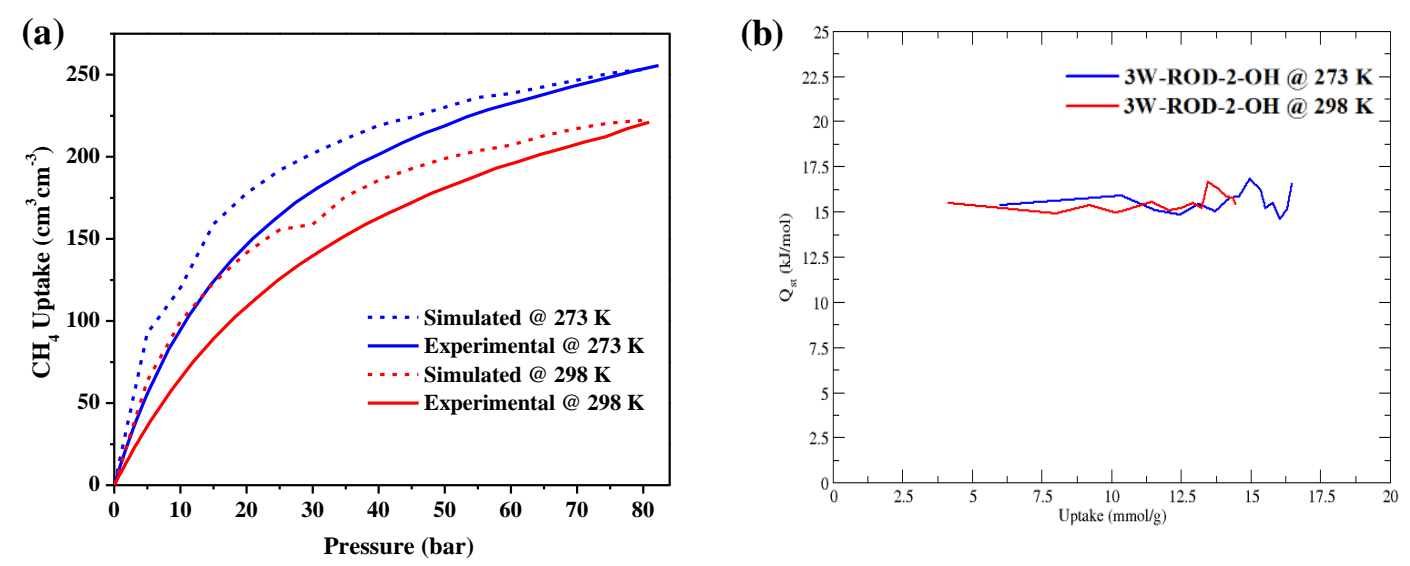

Figure S66. Comparison of simulated and experimental total methane adsorption isotherms (a) and simulated $Q_{\text {st }}$ for $3 \mathrm{~W}-\mathrm{ROD}-2-\mathrm{OH}$ (b).
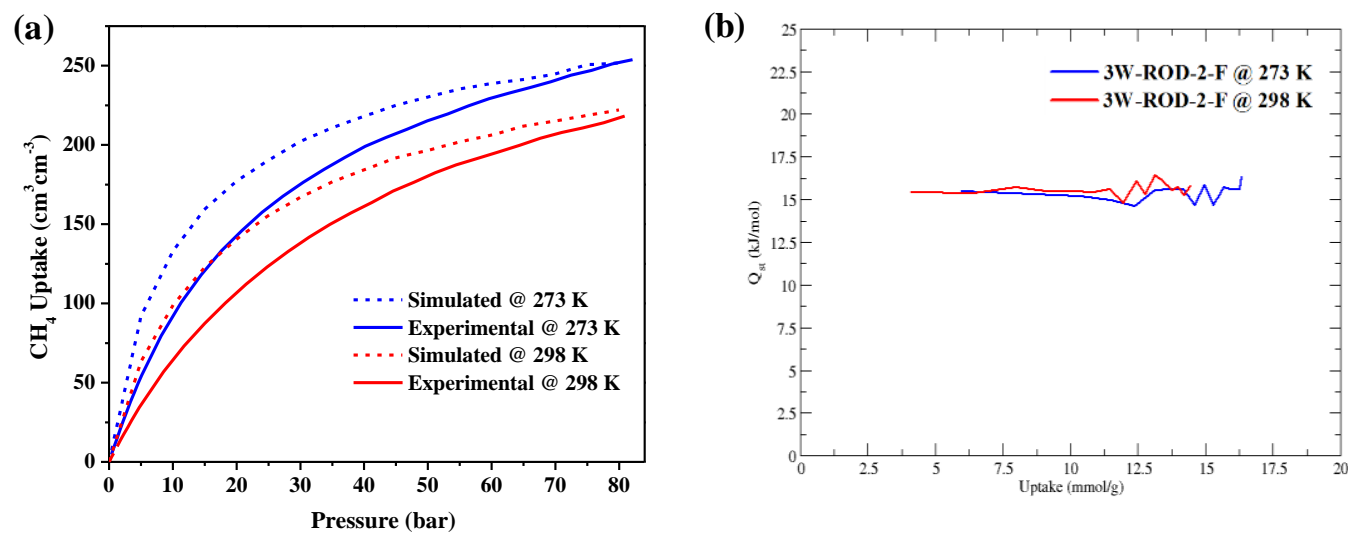

Figure S67. Comparison of simulated and experimental total methane adsorption isotherms (a) and simulated $Q_{\text {st }}$ for $3 \mathrm{~W}-\mathrm{ROD}-2-\mathrm{F}$ (b).
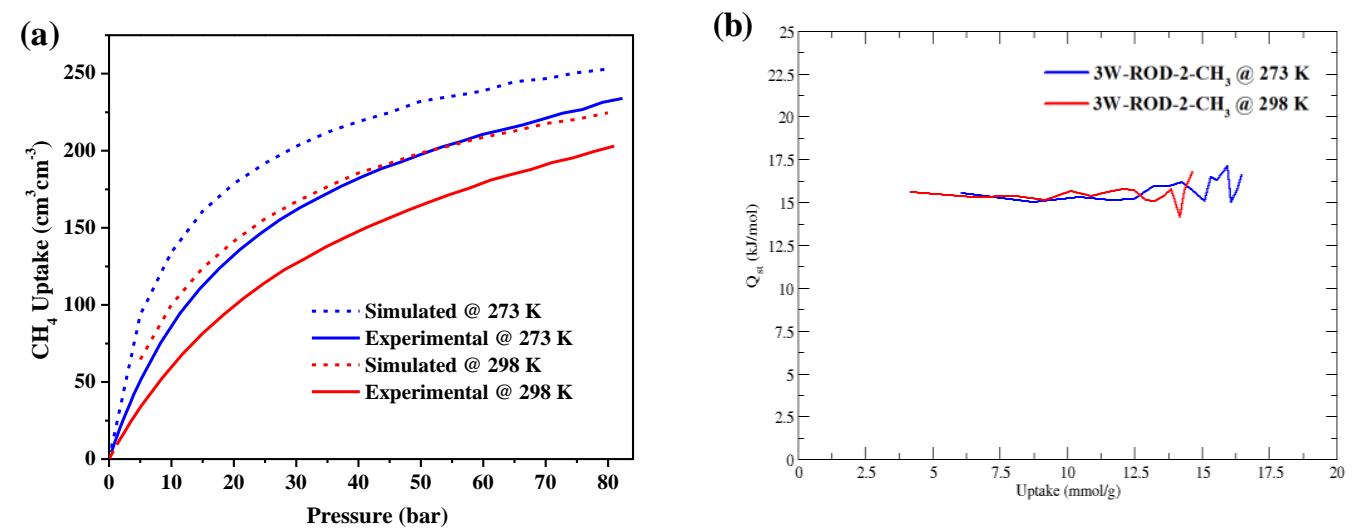

Figure S68. Comparison of simulated and experimental total methane adsorption isotherms (a) and simulated $Q_{\text {st }}$ for $3 \mathrm{~W}-\mathrm{ROD}-2-\mathrm{CH}_{3}$ (b). 


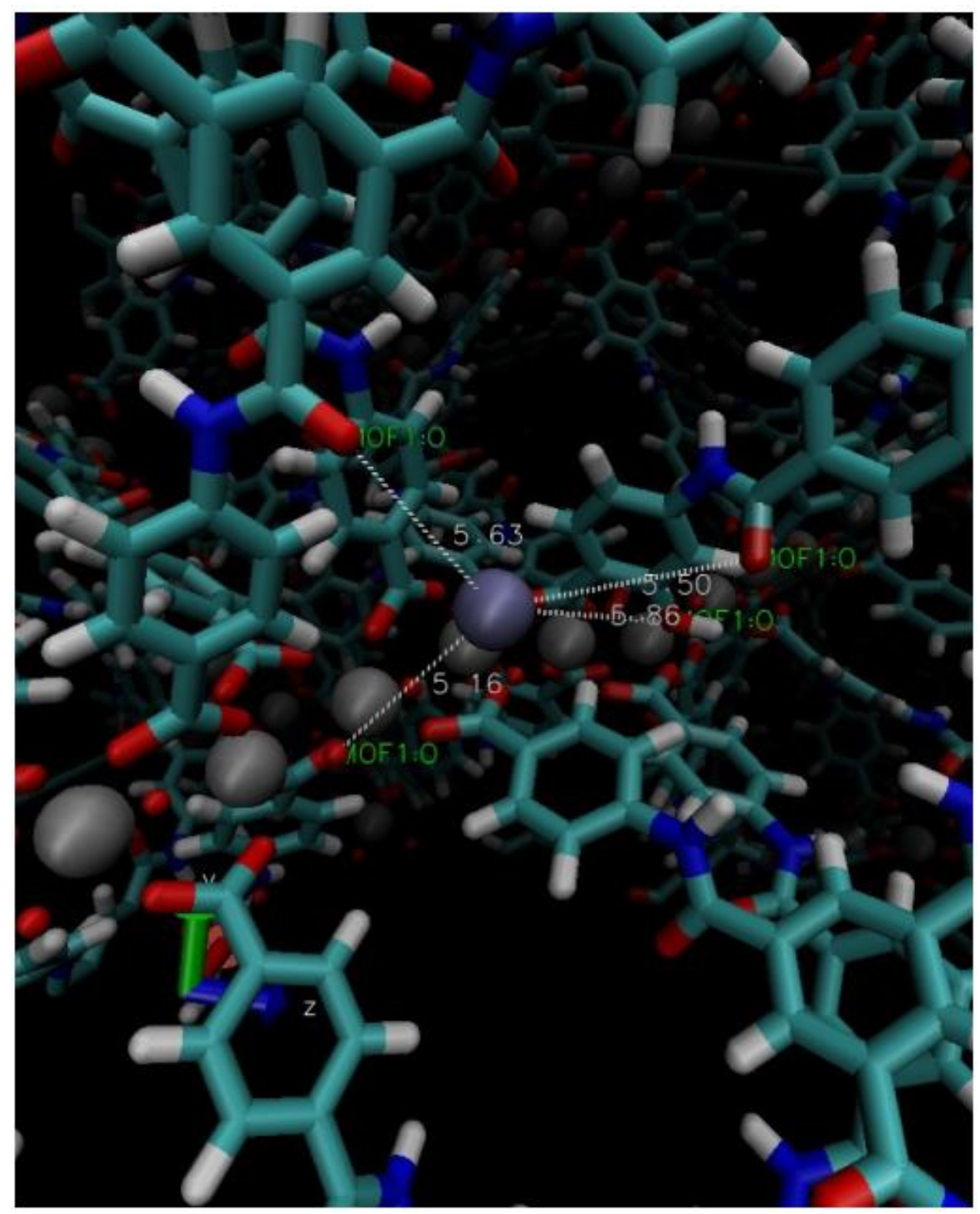

Figure S69. The van der Waals radius of methane was used to calculate binding site information for all variants of the MOF. Simulated annealing simulations started at $600 \mathrm{~K}$ and exponentially cooled to approximately $1 \mathrm{~K}$. Final binding energy for $3 \mathrm{~W}$ ROD-2-OH was $-23.045 \mathrm{~kJ} / \mathrm{mol}$. Binding interaction distances: $5.63 \AA$ for top left $\mathrm{O}$, $5.16 \AA$ for bottom left $\mathrm{O}$ on the In-O backbone, $5.50 \AA$ for top right $\mathrm{O}$, and $5.86 \AA$ for -OH group. Average interaction distance: $5.54 \AA$. 


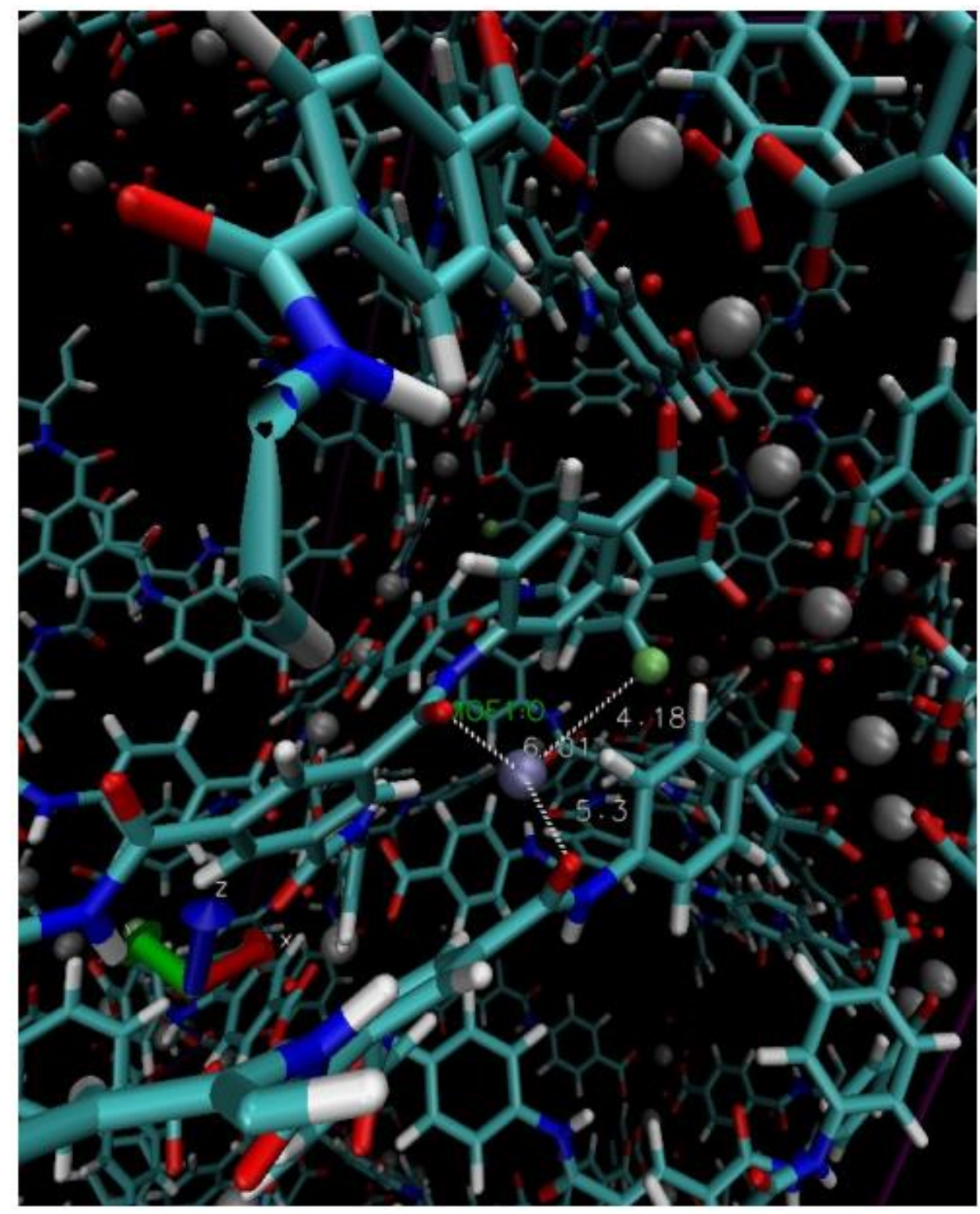

Figure S70. Final energy: $-20.004 \mathrm{~kJ} / \mathrm{mol}$ for $3 \mathrm{~W}-\mathrm{ROD}-2-\mathrm{F}$. Interaction distances: $6.01 \AA$ and 5.31 $\AA$ from left and right $\mathrm{O}$ atoms, respectively, and $4.18 \AA$ to $-\mathrm{F}$ group. Average interaction distance: $5.18 \AA$. 


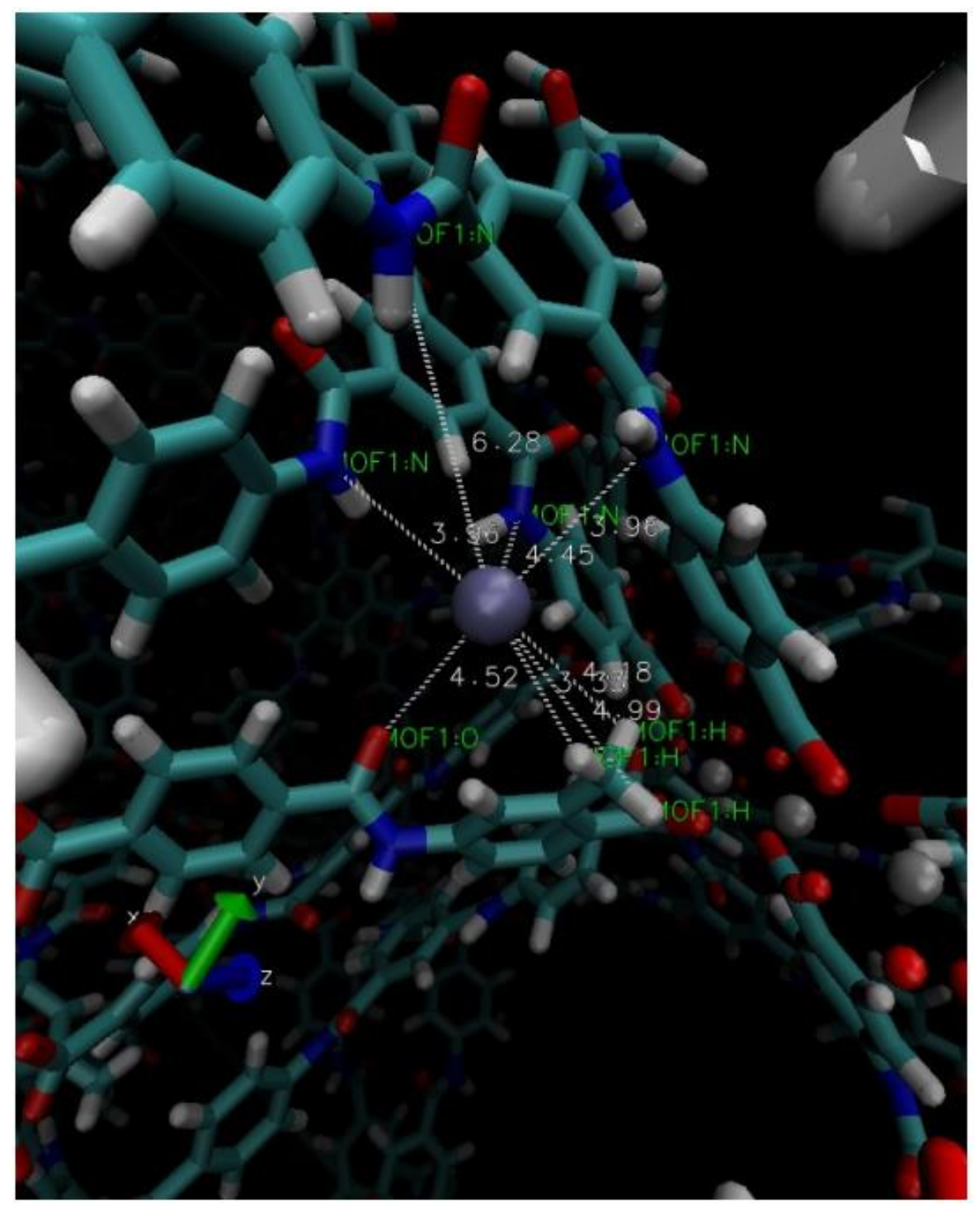

Figure S71. Final energy: $-21.101 \mathrm{~kJ} / \mathrm{mol}$ for $3 \mathrm{~W}-\mathrm{ROD}-2-\mathrm{CH}_{3}$. Interaction distances: $3.33 \AA$, $4.18 \AA$, and $4.99 \AA$ between the left, middle, and right $\mathrm{H}$ on the $-\mathrm{CH}_{3}$ group, respectively, interaction distances of $3.96 \AA, 6.28 \AA, 4.45 \AA$, and $3.96 \AA$ to N groups, respectively, and interaction distance of $4.52 \AA$ to $\mathrm{O}$ group. Average interaction distance: $4.46 \AA$. 


\section{Section 9. Single-Crystal X-Ray Crystallography}

Single-crystal X-ray diffraction data for 3W-ROD-1 was collected on a Bruker D8 venture diffractometer $(\mathrm{Cu} / K \alpha, \lambda=1.5418 \AA)$ at $243 \mathrm{~K}$. 3W-ROD-2-OH was collected on a synchrotron single crystal X-ray diffraction (SSCXRD) at $293 \mathrm{~K}$. Indexing was performed using APEX2 (Difference Vectors method). ${ }^{44}$ Data integration and reduction were performed using SaintPlus 6.01. ${ }^{45}$ Absorption correction was performed by multi-scan method implemented in SADABS. ${ }^{46}$ Space groups were determined using XPREP implemented in APEX2. The structures were solved by direct methods and refined with full-matrix least squares technique using the SHELXT package or refined using SHELXL-2014 (full-matrix least-squares on $\mathrm{F}^{2}$ ) contained in Olex2. ${ }^{47}$ Non-hydrogen atoms were refined with anisotropic displacement parameters during the final cycles. Hydrogen atoms were located at geometrically calculated positions to their carrier atoms and refined with isotropic thermal parameters included in the final stage of the refinement. For all compounds, the contributions of heavily disordered solvent molecules were treated as diffuse using Squeeze procedure implemented in Platon program. ${ }^{48}$ Crystal data and refinement conditions are shown in Tables S6-S7. 
Table S6. Crystal data and structure refinement for 3W-ROD-1.

\begin{tabular}{|c|c|}
\hline Identification code & 3W-ROD-1 \\
\hline Empirical formula & $\mathrm{C}_{90} \mathrm{H}_{49} \mathrm{In}_{5} \mathrm{~N}_{9} \mathrm{O}_{34}$ \\
\hline Formula weight & 2374.48 \\
\hline Temperature/K & 243.0 \\
\hline Crystal system & trigonal \\
\hline Space group & $R-3$ \\
\hline \multirow[t]{3}{*}{ Unit cell dimensions } & $a=41.8064(18) \AA \quad \alpha=90^{\circ}$ \\
\hline & $b=41.8064(18) \AA \quad \beta=90^{\circ}$ \\
\hline & $c=71.364(4) \AA \quad \gamma=120^{\circ}$ \\
\hline Volume $/ \AA^{3}$ & $108018(11)$ \\
\hline$Z$ & 18 \\
\hline$\rho_{\text {calc }} \mathrm{g} / \mathrm{cm}^{3}$ & 0.657 \\
\hline$\mu / \mathrm{mm}^{-1}$ & 4.083 \\
\hline $\mathrm{F}(000)$ & 21042.0 \\
\hline Crystal size $/ \mathrm{mm}^{3}$ & $0.15 \times 0.15 \times 0.12$ \\
\hline Radiation & $\mathrm{CuK} \alpha(\lambda=1.54178)$ \\
\hline Theta range for data collection ${ }^{\circ}$ & 3.714 to 101.47 \\
\hline Index ranges & $-35 \leq \mathrm{h} \leq 41,-38 \leq \mathrm{k} \leq 41,-71 \leq 1 \leq 70$ \\
\hline Reflections collected & 87547 \\
\hline Independent reflections & $25090\left[R_{\text {int }}=0.0671, R_{\text {sigma }}=0.0758\right]$ \\
\hline Data/restraints/parameters & $25090 / 410 / 1181$ \\
\hline Goodness-of-fit on $\mathrm{F}^{2}$ & 1.107 \\
\hline Final $R$ indexes $[\mathrm{I}>=2 \sigma(\mathrm{I})]$ & $R_{1}=0.0968, w R_{2}=0.2769$ \\
\hline Final $R$ indexes [all data] & $R_{1}=0.1067, w R_{2}=0.2887$ \\
\hline Largest diff. peak/hole / e $\AA^{-3}$ & $1.84 /-1.12$ \\
\hline
\end{tabular}


Table S7. Crystal data and structure refinement for 3W-ROD-2-OH.

\begin{tabular}{|c|c|}
\hline Identification code & 3W-ROD-2-OH \\
\hline Empirical formula & $\mathrm{C}_{95} \mathrm{H}_{55} \mathrm{In}_{5} \mathrm{~N}_{9} \mathrm{O}_{35}$ \\
\hline Formula weight & 2456.58 \\
\hline Temperature/K & 293(2) \\
\hline Crystal system & trigonal \\
\hline Space group & $R-3$ \\
\hline \multirow[t]{3}{*}{ Unit cell dimensions } & $a=42.190(6) \AA$ \\
\hline & $b=42.190(6) \AA \quad \beta=90^{\circ}$ \\
\hline & $c=71.140(14) \AA \quad \gamma=120^{\circ}$ \\
\hline Volume $/ \AA^{3}$ & 109664(38) \\
\hline$Z$ & 18 \\
\hline$\rho_{\text {calc }} \mathrm{g} / \mathrm{cm}^{3}$ & 0.670 \\
\hline$\mu / \mathrm{mm}^{-1}$ & 0.625 \\
\hline $\mathrm{F}(000)$ & 21834.0 \\
\hline Crystal size $/ \mathrm{mm}^{3}$ & $0.150 \times 0.100 \times 0.100$ \\
\hline Radiation & Synchrotron $(\lambda=0.77491)$ \\
\hline Theta range for data collection ${ }^{\circ}$ & 2.51 to 47.6 \\
\hline Index ranges & $-43 \leq \mathrm{h} \leq 40,-43 \leq \mathrm{k} \leq 43,-72 \leq 1 \leq 71$ \\
\hline Reflections collected & 113308 \\
\hline Independent reflections & $28290\left[R_{\text {int }}=0.0352, R_{\text {sigma }}=0.0367\right]$ \\
\hline Data/restraints/parameters & $28290 / 8 / 606$ \\
\hline Goodness-of-fit on $\mathrm{F}^{2}$ & 1.888 \\
\hline Final $R$ indexes $[\mathrm{I}>=2 \sigma(\mathrm{I})]$ & $R_{1}=0.1151, w R_{2}=0.4233$ \\
\hline Final $R$ indexes [all data] & $R_{1}=0.1227, w R_{2}=0.4506$ \\
\hline Largest diff. peak/hole / e $\AA^{-3}$ & $0.68 /-0.36$ \\
\hline
\end{tabular}




\section{Section 10. References.}

(1) Song, X.; Zou, Y.; Liu, X.; Oh, M.; Lah, M. S. A Two-Fold Interpenetrated (3,6)Connected Metal-Organic Framework with Rutile Topology Showing a Large Solvent Cavity. New J. Chem. 2010, 34, 2396-2399.

(2) Ma, J.; Kalenak, A. P.; Wong-Foy, A. G.; Matzger, A. J. Rapid Guest Exchange and Ultra-Low Surface Tension Solvents Optimize Metal-Organic Framework Activation. Angew. Chem., Int. Ed. 2017, 56, 14618-14621.

(3) Lo, S.-H.; Chien, C.-H.; Lai, Y.-L.; Yang, C.-C.; Lee, J. J.; Raja, D. S.; Lin, C.-H., A Mesoporous Aluminium Metal-Organic Framework with $3 \mathrm{~nm}$ Open Pores. J. Mater. Chem. A. 2013, 1, 324-329.

(4) Choi, H. J.; Dinca, M.; Long, J. R. Broadly Hysteretic $\mathrm{H}_{2}$ Adsorption in the Microporous Metal-Organic Framework Co(1,4-Benzenedipyrazolate). J. Am. Chem. Soc. 2008, 130, 78487850 .

(5) Mason, J. A.; Veenstra, M.; Long, J. R. Evaluating Metal-Organic Frameworks for Natural Gas Storage. Chem. Sci. 2014, 5, 32-51.

(6) Deng, H.; Grunder, S.; Cordova, K. E.; Valente, C.; Furukawa, H.; Hmadeh, M.; Gandara, F.; Whalley, A. C.; Liu, Z.; Asahina, S.; Kazumori, H.; O'Keeffe, M.; Terasaki, O.; Stoddart, J. F.; Yaghi, O. M. Large-Pore Apertures in a Series of Metal-Organic Frameworks. Science 2012, 336, 1018-1023.

(7) Catarineu, N. R.; Schoedel, A.; Urban, P.; Morla, M. B.; Trickett, C. A.; Yaghi, O. M. Two Principles of Reticular Chemistry Uncovered in a Metal Organic Framework of Heterotritopic Linkers and Infinite Secondary Building Units. J. Am. Chem. Soc. 2016, 138, 10826-10829.

(8) Du, M.; Chen, M.; Yang, X.-G.; Wen, J.; Wang, X.; Fang, S.-M.; Liu, C.-S. A ChannelType Mesoporous In(III)-Carboxylate Coordination Framework with High Physicochemical Stability for Use as an Electrode Material in Supercapacitors. J. Mater. Chem. A. 2014, 2, 9828-9834.

(9) Kundu, T.; Shah, B. B.; Bolinois, L.; Zhao, D. Functionalization-Induced Breathing Control in Metal-Organic Frameworks for Methane Storage with High Deliverable Capacity. Chem. Mater. 2019, 31, 2842-2847.

(10) Volkringer, C.; Loiseau, T.; Haouas, M.; Taulelle, F.; Popov, D.; Burghammer, M.; Riekel, C.; Zlotea, C.; Cuevas, F.; Latroche, M.; Phanon, D.; Knoefelv, C.; Llewellyn, P. L.; Ferey, G. Occurrence of Uncommon Infinite Chains Consisting of Edge-Sharing Octahedra in a Porous Metal Organic Framework-Type Aluminum Pyromellitate $\mathrm{Al}_{4}(\mathrm{OH})_{8}\left[\mathrm{C}_{10} \mathrm{O}_{8} \mathrm{H}_{2}\right]$ (MIL-120): Synthesis, Structure, and Gas Sorption Properties. Chem. Mater. 2009, 21, $5783-$ 5791.

(11) Devic, T.; Serre, C.; Audebrand, N.; Marrot, J.; Ferey, G. MIL-103, a 3-D LanthanideBased Metal Organic Framework with Large One-Dimensional Tunnels and a High Surface Area. J. Am. Chem. Soc. 2005, 127, 12788-12789.

(12) Tu, T. N.; Nguyen, H. T. D.; Nhung Thi, T. Tailoring the Pore Size and Shape of the One-Dimensional Channels in Iron-Based MOFs for Enhancing the Methane Storage Capacity. Inorg. Chem. Front. 2019, 6, 2441-2447.

(13) Guillerm, V.; Ragon, F.; Dan-Hardi, M.; Devic, T.; Vishnuvarthan, M.; Campo, B.; Vimont, A.; Clet, G.; Yang, Q.; Maurin, G.; Ferey, G.; Vittadini, A.; Gross, S.; Serre, C. A Series of Isoreticular, Highly Stable, Porous Zirconium Oxide Based Metal-Organic Frameworks. Angew. Chem., Int. Ed. 2012, 51, 9267-9271.

(14) Stylianou, K. C.; Heck, R.; Chong, S. Y.; Bacsa, J.; Jones, J. T. A.; Khimyak, Y. Z.; Bradshaw, D.; Rosseinsky, M. J. A Guest-Responsive Fluorescent 3d Microporous MetalOrganic Framework Derived from a Long-Lifetime Pyrene Core. J. Am. Chem. Soc. 2010, 132, 4119-4130.

(15) He, Y.-P.; Tan, Y.-X.; Zhang, J. Stable Mg-Metal-Organic Framework (MOF) and Unstable Zn-MOF Based on Nanosized Tris((4-Carboxyl)Phenylduryl)Amine Ligand. Cryst. Growth Des. 2013, 13, 6-9. 
(16) Herm, Z. R.; Wiers, B. M.; Mason, J. A.; van Baten, J. M.; Hudson, M. R.; Zajdel, P.; Brown, C. M.; Masciocchi, N.; Krishna, R.; Long, J. R. Separation of Hexane Isomers in a Metal-Organic Framework with Triangular Channels. Science 2013, 340, 960-964.

(17) Duan, J.; Higuchi, M.; Horike, S.; Foo, M. L.; Rao, K. P.; Inubushi, Y.; Fukushima, T.; Kitagawa, S. High $\mathrm{CO}_{2} / \mathrm{CH}_{4}$ and $\mathrm{C}_{2}$ Hydrocarbons/ $/ \mathrm{CH}_{4}$ Selectivity in a Chemically Robust Porous Coordination Polymer. Adv. Funct. Mater. 2013, 23, 3525-3530.

(18) Reinsch, H.; Krueger, M.; Marrot, J.; Stock, N. First Keto-Functionalized Microporous Al-Based Metal-Organic Framework: $\mathrm{Al}(\mathrm{OH})\left(\mathrm{O}_{2} \mathrm{C}-\mathrm{C}_{6} \mathrm{H}_{4}-\mathrm{CO}-\mathrm{C}_{6} \mathrm{H}_{4}-\mathrm{CO}_{2}\right)$. Inorg. Chem. 2013, 52, 1854-1859.

(19) Krüger, M.; Inge, A. K.; Reinsch, H.; Li, Y.-H.; Wahiduzzaman, M.; Lin, C.-H.; Wang, S.-L.; Maurin, G.; Stock, N. Polymorphous Al-MOFs Based on V-Shaped Linker Molecules: Synthesis, Properties, and in Situ Investigation of Their Crystallization. Inorg. Chem. 2017, 56, 5851-5862.

(20) Colombo, V.; Galli, S.; Choi, H. J.; Han, G. D.; Maspero, A.; Palmisano, G.; Masciocchi, N.; Long, J. R. High Thermal and Chemical Stability in Pyrazolate-Bridged Metal-Organic Frameworks with Exposed Metal Sites. Chem. Sci. 2011, 2, 1311-1319.

(21) Rosi, N. L.; Kim, J.; Eddaoudi, M.; Chen, B. L.; O'Keeffe, M.; Yaghi, O. M., Rod Packings and Metal-Organic Frameworks Constructed from Rod-Shaped Secondary Building Units. J. Am. Chem. Soc. 2005, 127, 1504-1518.

(22) Li, L.; Wang, S.; Chen, T.; Sun, Z.; Luo, J.; Hong, M., Solvent-Dependent Formation of $\mathrm{Cd}(\mathrm{II})$ Coordination Polymers Based on a $\mathrm{C}_{2}$-Symmetric Tricarboxylate Linker. Cryst. Growth Des. 2012, 12, 4109-4115.

(23) Peng, Y.; Krungleviciute, V.; Eryazici, I.; Hupp, J. T.; Farha, O. K.; Yildirim, T., Methane Storage in Metal-Organic Frameworks: Current Records, Surprise Findings, and Challenges. J. Am. Chem. Soc. 2013, 135, 11887-11894.

(24) Gandara, F.; Furukawa, H.; Lee, S.; Yaghi, O. M., High Methane Storage Capacity in Aluminum Metal-Organic Frameworks. J. Am. Chem. Soc. 2014, 136, 5271-5274.

(25) Jiang, J.; Furukawa, H.; Zhang, Y.-B.; Yaghi, O. M., High Methane Storage Working Capacity in Metal-Organic Frameworks with Acrylate Links. J. Am. Chem. Soc. 2016, 138, 10244-10251.

(26) Furukawa, H.; Ko, N.; Go, Y. B.; Aratani, N.; Choi, S. B.; Choi, E.; Yazaydin, A. O.; Snurr, R. Q.; O'Keeffe, M.; Kim, J.; Yaghi, O. M., Ultrahigh Porosity in Metal-Organic Frameworks. Science 2010, 329, 424-428.

(27) Zhang, M.; Zhou, W.; Pham, T.; Forrest, K. A.; Liu, W.; He, Y.; Wu, H.; Yildirim, T.; Chen, B.; Space, B.; Pan, Y.; Zaworotko, M. J.; Bai, J., Fine Tuning of MOF-505 Analogues to Reduce Low-Pressure Methane Uptake and Enhance Methane Working Capacity. Angew. Chem., Int. Ed. 2017, 56, 11426-11430.

(28) Li, B.; Wen, H.-M.; Wang, H.; Wu, H.; Tyagi, M.; Yildirim, T.; Zhou, W.; Chen, B., A Porous Metal-Organic Framework with Dynamic Pyrimidine Groups Exhibiting Record High Methane Storage Working Capacity. J. Am. Chem. Soc. 2014, 136, 6207-6210.

(29) Wen, H.-M.; Li, B.; Li, L.; Lin, R.-B.; Zhou, W.; Qian, G.; Chen, B., A Metal-Organic Framework with Optimized Porosity and Functional Sites for High Gravimetric and Volumetric Methane Storage Working Capacities. Adv. Mater. 2018, 30,1704792.

(30) Alezi, D.; Belmabkhout, Y.; Suyetin, M.; Bhatt, P. M.; Weselinski, L. J.; Solovyeva, V.; Adil, K.; Spanopoulos, I.; Trikalitis, P. N.; Emwas, A.-H.; Eddaoudi, M., Mof Crystal Chemistry Paving the Way to Gas Storage Needs: Aluminum-Based soc-MOF for $\mathrm{CH}_{4}, \mathrm{O}_{2}$, and $\mathrm{CO}_{2}$ Storage. J. Am. Chem. Soc. 2015, 137, 13308-13318.

(31) (a) Zhao, D.; Yuan, D.; Yakovenko, A.; Zhou, H.-C., A Nbo-Type Metal-Organic Framework Derived from a Polyyne-Coupled Di-Isophthalate Linker Formed in Situ. Chem. Commun. 2010, 46, 4196-4198; (b) Shao, K.; Pei, J.; Wang, J.-X.; Yang, Y.; Cui, Y.; Zhou, W.; Yildirim, T.; Li, B.; Chen, B.; Qian, G., Tailoring the Pore Geometry and Chemistry in Microporous Metal-Organic Frameworks for High Methane Storage Working Capacity. Chem. Commun. 2019, 55, 11402-11405. 
(32) Yan, Y.; Kolokolov, D. I.; da Silva, I.; Stepanov, A. G.; Blake, A. J.; Dailly, A.; Manuel, P.; Tang, C. C.; Yang, S.; Schröder, M., Porous Metal-Organic Polyhedral Frameworks with Optimal Molecular Dynamics and Pore Geometry for Methane Storage. J. Am. Chem. Soc. 2017, 139, 13349-13360.

(33) Alezi, D.; Spanopoulos, I.; Tsangarakis, C.; Shkurenko, A.; Adil, K.; Belmabkhout, Y.; O'Keeffe, M.; Eddaoudi, M.; Trikalitis, P. N., Reticular Chemistry at Its Best: Directed Assembly of Hexagonal Building Units into the Awaited Metal-Organic Framework with the Intricate Polybenzene Topology, pbz-MOF. J. Am. Chem. Soc. 2016, 138, 12767-12770.

(34) Tu, B.; Diestel, L.; Shi, Z.-L.; Bandara, W. R. L. N.; Chen, Y.; Lin, W.; Zhang, Y.-B.; Telfer, S. G.; Li, Q., Harnessing Bottom-up Self-Assembly to Position Five Distinct Components in an Ordered Porous Framework. Angew. Chem., Int. Ed. 2019, 58, 5348-5353.

(35) Lin, J.-M.; He, C.-T.; Liu, Y.; Liao, P.-Q.; Zhou, D.-D.; Zhang, J.-P.; Chen, X.-M., A Metal-Organic Framework with a Pore Size/Shape Suitable for Strong Binding and Close Packing of Methane. Angew. Chem., Int. Ed. 2016, 55, 4674-4678.

(36) Liang, C.-C.; Shi, Z.-L.; He, C.-T.; Tang, J.; Zhou, H.-D.; Zhou, H.-L.; Lee, Y.; Zhang, Y.-B., Engineering of Pore Geometry for Ultrahigh Capacity Methane Storage in Mesoporous Metal-Organic Frameworks. J. Am. Chem. Soc. 2017, 139, 13300-13303.

(37) Spanopoulos, I.; Tsangarakis, C.; Klontzas, E.; Tylianakis, E.; Froudakis, G.; Adil, K.; Belmabkhout, Y.; Eddaoudi, M.; Trikalitis, P. N., Reticular Synthesis of HKUST-like tboMOFs with Enhanced $\mathrm{CH}_{4}$ Storage. J. Am. Chem. Soc. 2016, 138, 1568-1574.

(38) Chen, Z.; Li, P.; Anderson, R.; Wang, X.; Zhang, X.; Robison, L.; Redfern, L. R.; Moribe, S.; Islamoglu, T.; Gomez-Gualdron, D. A.; Yildirim, T.; Stoddart, J. F.; Farha, O. K., Balancing Volumetric and Gravimetric Uptake in Highly Porous Materials for Clean Energy. Science 2020, 368, 297-303.

(39) Franz, D. M.; Belof, J. L.; McLaughlin, K.; Cioce, C. R.; Tudor, B.; Hogan, A.; Laratelli, L.; Mulcair, M.; Mostrom, M.; Navas, A.; Stern, A. C.; Forrest, K. A.; Pham, T.; Space, B. MPMC and MCMD: Free High-Performance Simulation Software for Atomistic Systems. Adv. Theor. Simul. 2019, 2, 1900113.

(40) Peng, D.-Y.; Robinson, D. B. A New Two-Constant Equation of State. Ind. Eng. Chem. Fundam. 1976, 15, 59-64.

(41) Rappe, A. K.; Casewit, C. J.; Colwell, K. S.; Goddard, W. A.; Skiff, W. M. Uff, a Full Periodic Table Force Field for Molecular Mechanics and Molecular Dynamics Simulations. $J$. Am. Chem. Soc. 1992, 114, 10024-10035.

(42) Valiev, M.; Bylaska, E. J.; Govind, N.; Kowalski, K.; Straatsma, T. P.; Van Dam, H. J. J.; Wang, D.; Nieplocha, J.; Apra, E.; Windus, T. L.; de Jong, W. NWchem: A Comprehensive and Scalable Open-Source Solution for Large Scale Molecular Simulations. Comput. Phys. Commun. 2010, 181, 1477-1489.

(43) (a) Cioce, C. R.; McLaughlin, K.; Belof, J. L.; Space, B. A Polarizable and Transferable Phast $\mathrm{N}_{2}$ Potential for Use in Materials Simulation. J. Chem. Theory Comput. 2013, 9, 55505557. (b) Cioce, C. R.; Tudor, B.; McLaughlin, K.; Belof, J. L.; Space, B. "A PHAST Potential for Heterogeneous Simulation of Methane with Many-Body Interactions." Manuscript in preparation. (c) Pham, T.; Forrest, K. A.; Tudor, B.; Elsaidi, S. K.; Mohamed, M. H.; McLaughlin, K.; Cioce, C. R.; Zaworotko, M. J.; Space, B. Theoretical Investigations of $\mathrm{CO}_{2}$ and $\mathrm{CH}_{4}$ Sorption in an Interpenetrated Diamondoid Metal-Organic Material. Langmuir 2014, 30, 6454-6462.

(44) APEX2; Bruker AXS: Madison, WI, USA, 2010.

(45) SAINT, Data Reduction Software; Bruker AXS: Madison, WI, USA. 2009.

(46) Sheldrick, G. M. SADABS, Program for Empirical Adsorption Correction for Area Detector Data; University of Gottingen: Gottingen, Germany, 1996.

(47) (a) Sheldrick, G. M. SHELXT-Integrated Space-Group and Crystal-Structure Determination. Acta Crystallogr., Sect. A: Found. Adv. 2015, A71, 3-8. (b) Dolomanov, O. V.; Bourhis, L. J.; Gildea, R. J.; Howard, J. A. K.; Puschmann, H. Olex2: A Complete Structure Solution, Refinement and Analysis Program. J. Appl. Crystallogr. 2009, 42, 339-341. 
(48) (a) Van der Sluis, P.; Spek, A. L. BYPASS: An Effective Method for the Refinement of Crystal Structures Containing Disordered Solvent Regions. Acta Cryst. 1990, A46, 194-201. (b) Spek, A. L. Single-Crystal Structure Validation with the Program Platon. J. Appl. Crystallogr. 2003, 36, 7-13. (c) Spek, A. L. Structure Validation in Chemical Crystallography. Acta Crystallogr., Sect. D: Biol. Crystallogr. 2009, D65, 148-155. 